\title{
Amplifier Design for Underground Anomaly Detection Using Naturally Occurring Ultra Low Frequencies
}

\author{
Dakota James Kirby
}

Follow this and additional works at: https://researchrepository.wvu.edu/etd

\section{Recommended Citation}

Kirby, Dakota James, "Amplifier Design for Underground Anomaly Detection Using Naturally Occurring Ultra Low Frequencies" (2017). Graduate Theses, Dissertations, and Problem Reports. 5983.

https://researchrepository.wvu.edu/etd/5983

This Thesis is protected by copyright and/or related rights. It has been brought to you by the The Research Repository @ WVU with permission from the rights-holder(s). You are free to use this Thesis in any way that is permitted by the copyright and related rights legislation that applies to your use. For other uses you must obtain permission from the rights-holder(s) directly, unless additional rights are indicated by a Creative Commons license in the record and/ or on the work itself. This Thesis has been accepted for inclusion in WVU Graduate Theses, Dissertations, and Problem Reports collection by an authorized administrator of The Research Repository @ WVU. For more information, please contact researchrepository@mail.wvu.edu. 


\title{
Amplifier Design for Underground Anomaly Detection Using Naturally Occurring Ultra Low Frequencies
}

\author{
Dakota James Kirby
}

\author{
Thesis submitted to the \\ Benjamin M. Statler College \\ of Engineering and Mineral Resources \\ at West Virginia University \\ in partial fulfillment of the requirements \\ for the degree of \\ Master of Science \\ in
}

Electrical Engineering

Roy S. Nutter Jr., Ph.D., Chair

Matthew C. Valenti, Ph.D.

James Smith, Ph.D.

Lane Department of Computer Science and Electrical Engineering

Morgantown, West Virginia

2017

Keywords: Amplifiers, Anomaly, Low Frequency, Low Noise, Schuman Resonance, Void

Copyright 2017 Dakota James Kirby 


\section{ABSTRACT \\ Amplifier Design for Underground Anomaly Detection Using Naturally Occurring Ultra Low Frequencies}

\section{Dakota James Kirby}

Most high-frequency signals are unable to penetrate the Earth's surface and lower frequencies have a much higher chance of penetrating the surface. Ultra-low frequencies can be used to penetrate the Earth's crust, but the frequency and background noise are a large problem that prevents from detecting these low frequency signals. This thesis describes the design for a small and lightweight low-frequency amplifier that can receive low voltage signals and amplify them to a level that could be used to do further analysis. The proposed amplifier design was capable of handling inputs as low as $1 \mu \mathrm{V}$ and amplifying them to a level of $1 \mathrm{mV}$. This thesis also describes how the amplifier can be tested along with the rest of a low-frequency detection system. 


\section{ACKNOWLEDGEMENTS}

I would like to express my thanks to my advisor Dr. Roy S. Nutter, Jr. for his assistance. I am extremely grateful to him for his guidance, encouragement and for the time he spent reviewing this paper and helping me further my education. I would also like to thank my family for always encouraging me to keep working and supporting me to accomplish my goals. Finally, I am extremely grateful to my wife, Chelsea, for sticking with me through this whole process and never giving up on me. 


\section{TABLE OF CONTENTS}

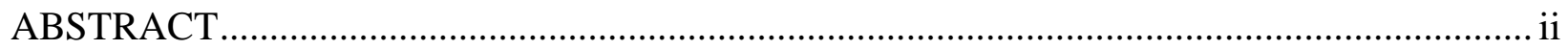

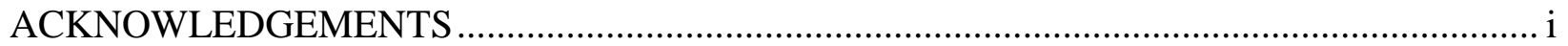

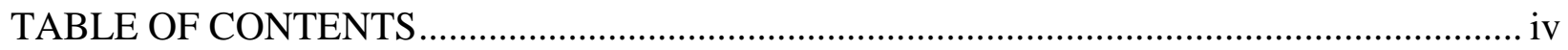

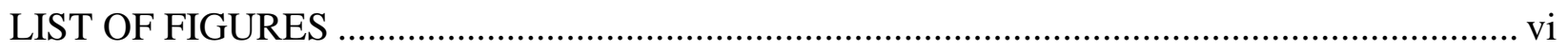

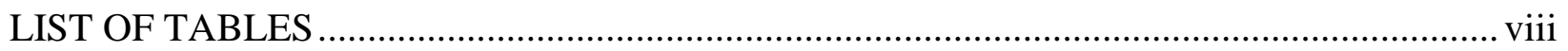

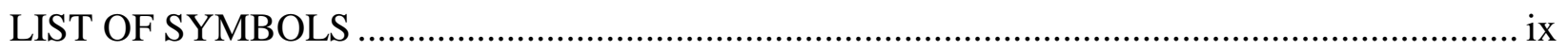

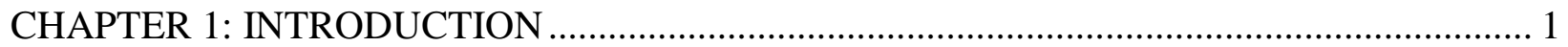

1.1 The Need for Underground Anomaly Detection ............................................. 1

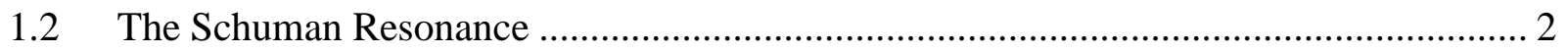

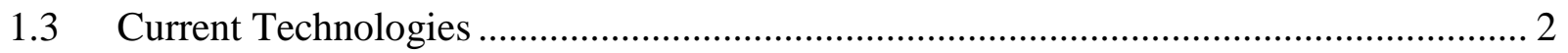

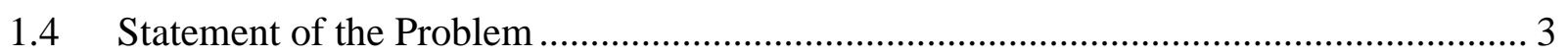

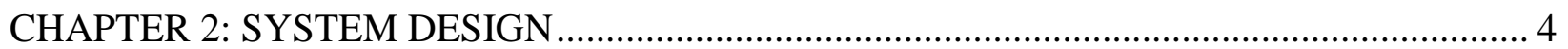

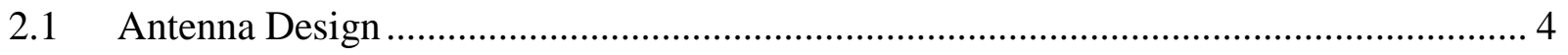

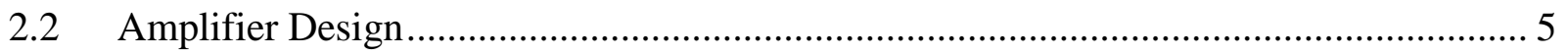

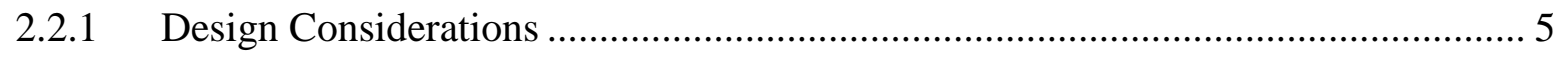

2.2.2 Amplifier components and final design .................................................. 11

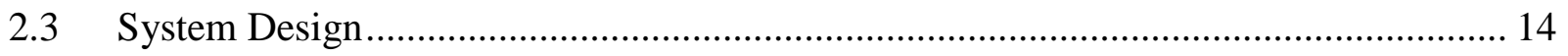

CHAPTER 3: SIMULATION OF AMPLIFIER AND ANTENNA …................................... 18

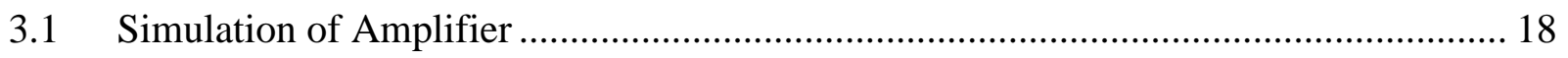

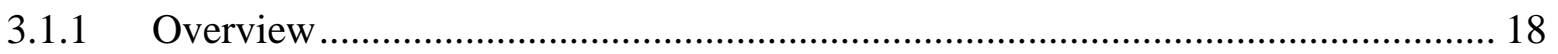

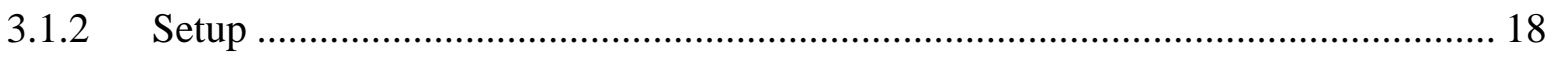

3.1.3 Small Signal Analysis ............................................................................... 19

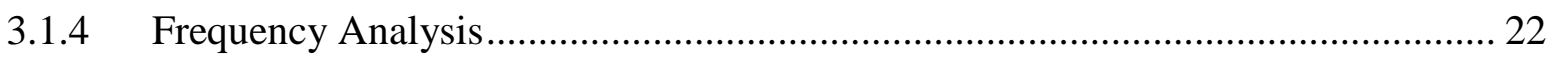

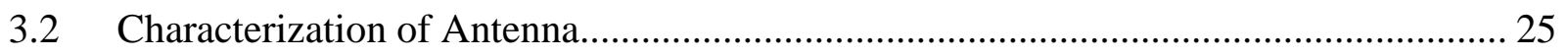

CHAPTER 4: BENCH TESTING AND ANALYSIS......................................................... 30

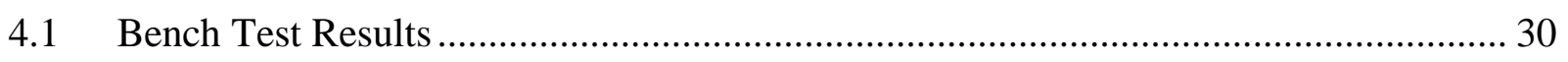

4.1.1 Amplifier Bench Test................................................................................... 30

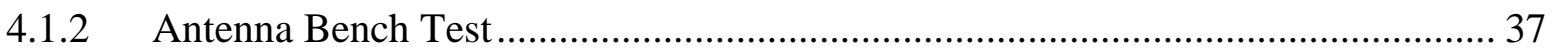

4.1.3 System Bench Test...................................................................................... 40

4.2 Analysis of Bench Test Results...................................................................... 42

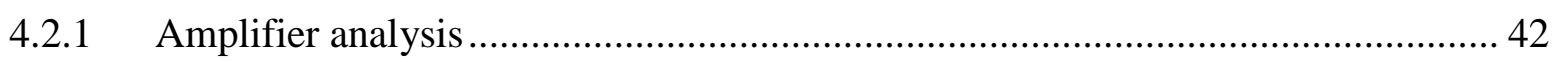

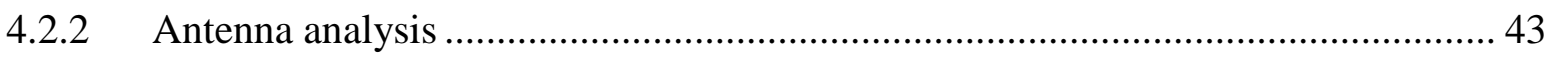




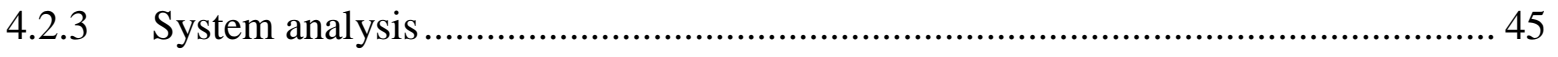

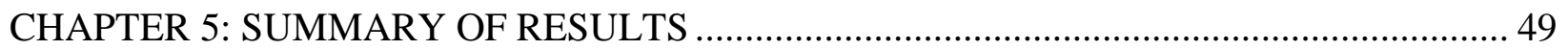

5.1 Summary of Simulation of Amplifier Results.............................................................. 49

5.2 Summary of Characterization of Antenna Results ........................................................ 49

5.3 Summary of Bench Test Results and Analysis ............................................................. 49

CHAPTER 6: CONCLUSION AND FUTURE WORK …………………………………......... 51

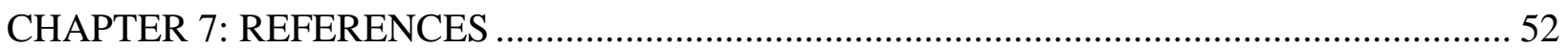




\section{LIST OF FIGURES}

Figure 1 - Hardware filtering and amplifier attempt overview ............................................... 6

Figure 2 - Hardware filtering and amplifier schematic................................................. 6

Figure 3 - Hardware filtering and amplifier frequency response ......................................... 7

Figure 4 - Typical Operational Amplifier Circuit [11] ..................................................... 8

Figure 5 - Operational Amplifiers used to build instrumentation amplifier [12] ...................... 9

Figure 6 - Typical Instrumentation Amplifier Design [11] ..................................................... 10

Figure 7 - Final Amplifier Design in LTSpice..................................................................... 11

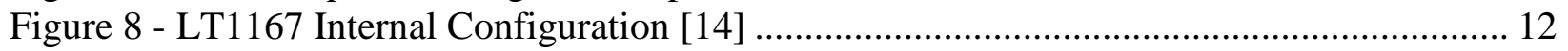

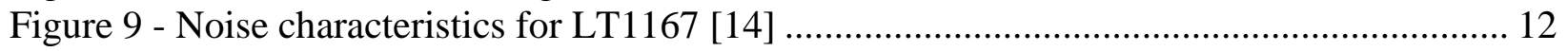

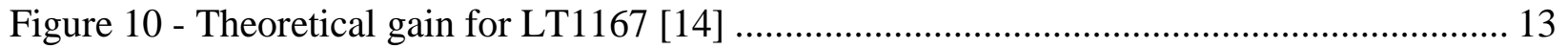

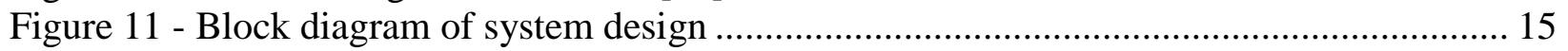

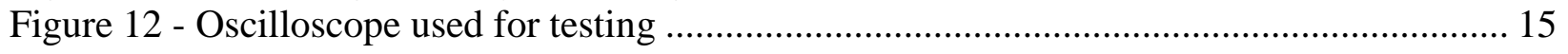

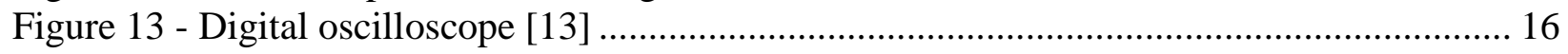

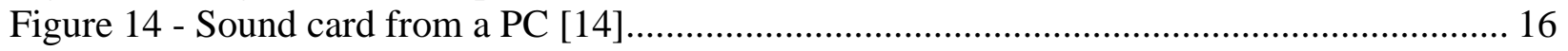

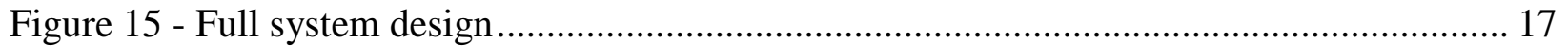

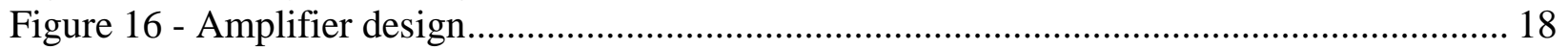

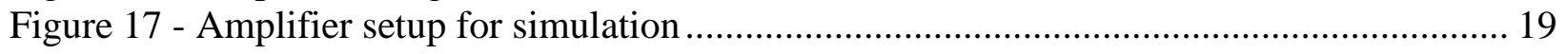

Figure 18 - Input signal for simulation ......................................................................... 20

Figure 19 - Output signal from simulation ......................................................................... 21

Figure 20- Output (red) signal compared to input (green) signal ......................................... 22

Figure 21 - Input frequency sweep ......................................................................... 23

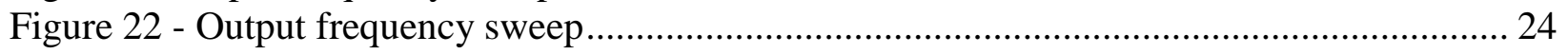

Figure 23 - Output sweep (red) compared to input sweep (green) ........................................ 25

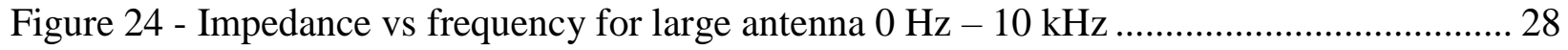

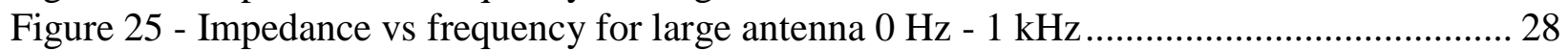

Figure 26 - Impedance vs frequency for small antenna $0 \mathrm{~Hz}-10 \mathrm{kHz}$.................................. 29

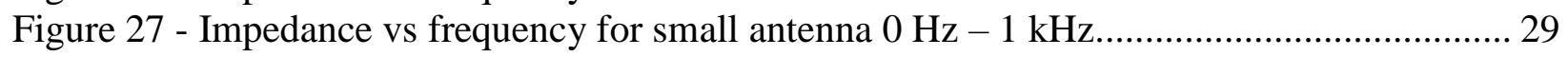

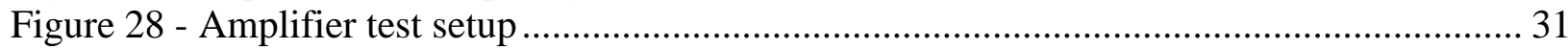

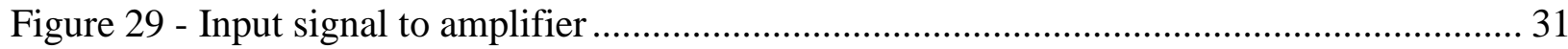

Figure 30 - Saturated output signal from amplifier ..................................................... 32

Figure 31 - Voltage divider circuit................................................................................... 33

Figure 32 - Revised amplifier test setup as block diagram .................................................. 34

Figure 33 - Output signal from amplifier with new test setup ............................................ 35

Figure 34 - Input signal before voltage division (black) and output from amplifier (red) .......... 35

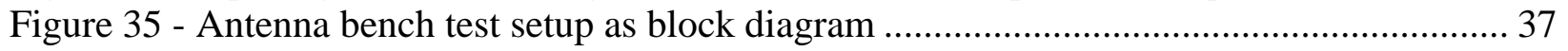

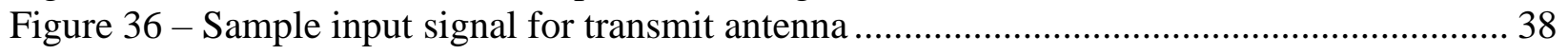

Figure 37 - Received signal by receive antenna ............................................................. 38

Figure 38 - System bench test setup as block diagram ....................................................... 40

Figure 39 - Spectrum lab output, showing waterfall display and oscilloscope functionality ....... 41

Figure 40 - Amplifier bench test frequency vs gain plot .................................................. 42

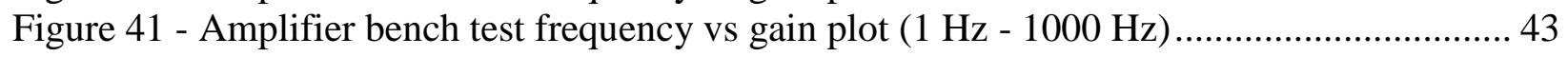

Figure 42 - Antenna frequency vs gain plot full frequency range ........................................... 44

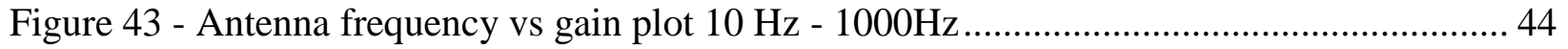

Figure 44 - Sound card frequency vs gain full range plot.................................................. 45 


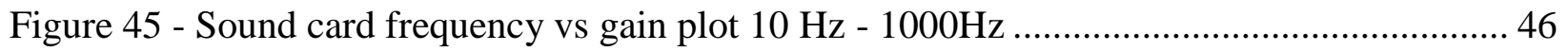

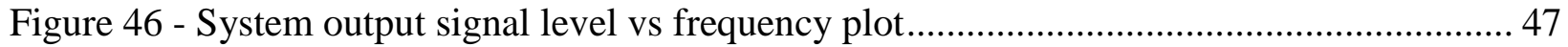

Figure 47 - System output signal level vs frequency plot $1 \mathrm{~Hz}-1500 \mathrm{~Hz}$............................. 47 


\section{LIST OF TABLES}

Table 1 - Design goals overview ..................................................................................... 4

Table 2 - Antenna Design Parameters ................................................................................... 5

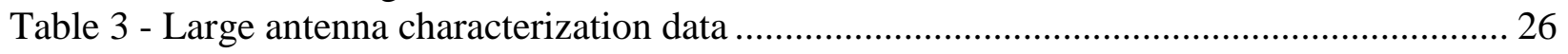

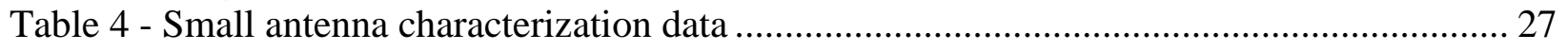

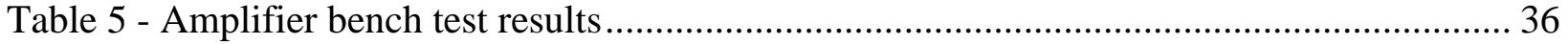

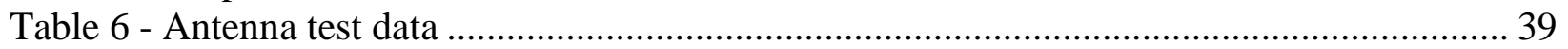

Table 7 - System test data ................................................................................................... 41

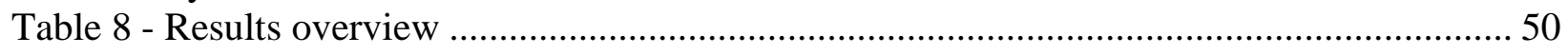




\section{LIST OF SYMBOLS}

DC - direct current

GPR - ground penetrating radar

In-amp - instrumentation amplifier

Op-amp - operational amplifier

$\mathrm{PC}$ - personal computer

A/D - analog-to-digital converter

AWG - American wire gauge 


\section{CHAPTER 1: INTRODUCTION}

\subsection{The Need for Underground Anomaly Detection}

Underground object or anomaly detection refers to the ability to detect or view objects that are buried beneath the Earth's surface and are not visible to the naked eye. The goal of a system that performs this detection is to be as non-invasive as possible while still being able to view in some manner what is present under the Earth's surface. The potential for a device that is accurately capable of this at long ranges/depths would be invaluable. Cave/mine detection, excavation for archeological digs, landmine detection and utility detection will find a use for this technology. Some current challenges posed in those areas are discussed below in explaining how a system like this could solve some current issues in these areas.

Underground utilities play a huge role in the everyday lives of people across the world. Without such utilities, including running water, several things in today's modern society would not be possible. Protecting these assets is crucial daily. Problems with these systems that cannot be seen from above the Earth's surface cannot be ignored and must be dealt with in a timely manner as to not cause larger issues [1]. Underground anomaly detection would allow different city, state and even federal organizations to detect these situations early and plan corrective actions before a catastrophic failure happens and residents of a town or state go without utilities for an extended amount of time.

Along with underground utilities, there is a multitude of other infrastructure uses for this system. Some of those uses include examining the ground in an area before construction is started to ensure that the ground is solid and there are no unknown cavities or voids below it. This technology would have uses in the mining field for emergency response, as well. With the ability to detect voids underground, emergency personnel would be able to locate trapped miners quickly and more efficiently. It could also be used to help miners find potential voids and cavities above and beneath them to help prevent mine collapses.

Another use for this technology is landmine detection. Currently, most land mine detection and removal techniques are crude and dangerous. If the land mines are non-metallic, they are also more difficult to detect [2]. With this technology, non-metallic land mines would be easier to detect, thus making them easier to navigate and remove.

With all the positive results that could be obtained, there are also several challenges that will be faced by developing this technology. Many factors play into the success of a system like this, including, the moisture of the soil at the time of measurement (the damper the soil the more likely the system will have issues), multiple items in a small area creating results that are almost unreadable, or where there is randomness in interference from the air/ground that results in unreadable outputs [3]. Considering all these factors is rather difficult, and all systems that currently exist are susceptible to at least one or more of these factors. Creating new technology that strives to minimize these properties while still being light and portable would be an enormous step forward for underground anomaly detection. 


\subsection{The Schuman Resonance}

There are constant signals that exist all around that can be received by specialized equipment at any point in time. Some of these signals are stronger and more frequent than others. The Schuman resonance is one of these signals. These signals are generated by lightning strikes across the Earth, and these strikes have frequencies that resonate between the Earth and the ionosphere, which creates a signal that can be measured with advanced equipment [4]. The frequency of this signal is approximately $7.8 \mathrm{~Hz}$ with several harmonics following at $14.1,20.3,26.4$, and 32.5 . There are even more harmonics past those, but these five are the notable frequencies in terms of signal strength [5]. Due to the ionosphere cavity, the signals are greatly attenuated over time resulting in very weak signals. The signal strength ranges are estimated to be in the range of $0.0005 \mu \mathrm{T}-0.001$ $\mu \mathrm{T}$. These signals were used as the basis for the amplifier design to avoid using a transmitter to help reduce the size and power requirements of the system.

\subsection{Current Technologies}

There are several systems that currently exist that are capable of anomaly/object detection to varying degrees. The most frequent of all the systems that are available is the ground penetrating radar, GPR. The major drawback to this type of system is that most objects cannot be detected at more than just a couple centimeters [6]. The most common technologies that are currently used and a short description of them are listed below.

\section{- Ground penetrating radar}

Ground penetrating radar, or GPR, is one of the more versatile methods that currently exist [7] and typically work by transmitting a high-frequency signal with a bandwidth of $1-4 \mathrm{GHz}$ (for landmine detection) and then receiving the results that are reflected. An image of what is in the ground can then be produced. The system can be very successful when optimized for certain goals, but the system typically must be mounted or pulled on a vehicle due to its size/power requirements [2]. Another one of the major drawbacks to GPR is that it typically needs a high bandwidth to improve the results, but this then requires higher frequencies which result in higher attenuation, making the results less and less reliable [7].

\section{- Acoustic wave detection}

Acoustic wave detection is still a newer technology and is not quite fully developed. Acoustic wave detection employs some variation of a loudspeaker that blasts the ground with lowfrequency acoustic signals [2] in the range of $60 \mathrm{~Hz}-200 \mathrm{~Hz}$ [8]. The transmitted signal would then proceed through the porous ground and be reflected up by anything that was non-porous. This reflected signal would then have to be received by a microphone which could then be recorded and used in future processing steps [9].

- Forward-looking infrared 
Forward-looking infrared has primarily been used to detect land mines. This system takes advantage of the way the soil directly above the anomaly heats and cools differently than the

soil around it. As the name implies, it is also able to detect these anomalies across a field of view ahead and not just below the sensor itself. [2]

Common features of these systems are that they all require some form of a transmitter to be able to detect anomalies, and they also have a very limited range. A requires more power to operate the device. A system that does not rely on a transmitter and instead relies on a naturally occurring frequency would be smaller, lighter and more power efficient. A portable system that can detect anomalies will meet the demands of a variety of users.

\subsection{Statement of the Problem}

This thesis documents an investigation of the design for an amplifier that can assist in detecting anomalies, voids and other foreign objects that might be present in the ground buried. The system will only use naturally occurring extremely low frequencies to determine if an object is present in the ground. In this work, a complete hardware system will be designed, built and tested with a primary focus on the amplifier. The system will use common technologies and combine them in ways that will help to detect extremely low-frequency signals. 


\section{CHAPTER 2: SYSTEM DESIGN}

The goal of the system was to analyze low power, low-frequency signals. There are many things that needed to be taken into consideration to achieve a system that could receive signals at such low levels. The noise generated by the antenna and the amplifier circuit were two of the biggest concerns. If the noise was too loud, then the signal would have been unrecoverable and would have been of no use. Software filtering and amplifying were also under consideration to help assist with recovering the signal from the noise. For amplification, the signal needed to be high enough to be received by a typical "line in" on a PC soundcard for further processing on a computer and displayed for signal visualization. If the signal could be received through a PC soundcard, then there was potential to make the system portable which was another goal of the system.

To achieve optimal results, the problem was broken down into two parts: the antenna and the amplifier. An antenna design was chosen that could receive extremely low frequencies around $7 \mathrm{~Hz}$ and could also receive extremely low signal strength, somewhere around $1 \mu \mathrm{V}$. This was not an easy task and was carefully designed. For the amplifier, similar design parameters were considered. When selecting parts, the frequency range and signal level were considered to achieve a design that could receive such small signals and would not filter out low frequencies. In the following sections, there are detailed considerations for each design component and then a final design selection for use in the system. The following table shows the major design criteria that were used for the system. The results column was completed after all testing was performed and is included in the summary section.

\begin{tabular}{|l|l|l|}
\hline Design Parameter & Goals & Result \\
\hline Frequency Range & $5 \mathrm{~Hz}-100 \mathrm{~Hz}$ & \\
\hline Amplifier Gain & $60 \mathrm{~dB}$ Gain at $<1000 \mathrm{~Hz}$ & \\
\hline \multicolumn{2}{|c|}{ Table $1-$ Design goals overview } \\
&
\end{tabular}

\subsection{Antenna Design}

The first thing the system needed was an antenna so that it could receive the signals. Without a proper antenna, not much could have been accomplished with the system. For the antenna, the system needed to use something pre-existing or something that would be simple to construct as trying to design a custom antenna was not the focus. The antenna for this application needed to have a small form factor so that it could be moved by one person without assistance from anyone or anything. It also needed to be lightweight, so that one person would be able to carry it or potentially push it on some sort of cart. To achieve this, many different antenna types were considered, but the final design settled on was a loop antenna. Typically loop antennas are not used for such low frequencies, but when a ferrite core is used instead of the typical air core, the frequency range of the antenna was much lower. The final design for the antenna consisted of a ferrite core with 18 AWG wire wrapped around the core. The wire was wound around the core back and forth starting at one end and winding to the other and then proceeding to wind the wire back the other direction, until the desired amount of winding was placed on the antenna. Two antennas were built to be used with the amplifier with the following specifications. 


\begin{tabular}{|l|l|l|l|l|}
\hline Antenna & $\begin{array}{l}\text { Length of } \\
\text { Wire (feet) }\end{array}$ & $\begin{array}{l}\text { Number of } \\
\text { Winding Layers }\end{array}$ & $\begin{array}{l}\text { Number of } \\
\text { Windings Per Layer }\end{array}$ & $\begin{array}{l}\text { Total number of } \\
\text { windings }\end{array}$ \\
\hline Large Coil & $\sim 700$ & 17 & $\sim 120$ & $\sim 2400$ \\
\hline Small Coil & $\sim 300$ & 4 & $\sim 120$ & $\sim 480$ \\
\hline
\end{tabular}

Table 2 - Antenna Design Parameters

The core inside both antennas had a diameter 1.02 inches and the length of the core was 6 inches with the windings only taking up 5 inches of that centered on the coil. The ferrite cores also have a permeability of 2000 [10]. With this design, the goal was to be able to receive a signal well enough that the amplifier could assist in boosting the signal to a useable level. The antennas are characterized in a later section.

\subsection{Amplifier Design}

There were many things to consider for the amplifier design. In the following sections, several aspects of the amplifier design will be examined to better determine the proper design for the amplifier. The sections consider various gain stages, amplification versus filtering, digital signal amplification, noise problems, and different kinds of amplifiers that could have been used.

\subsubsection{Design Considerations}

For the system, the amplifier was the most important part. Without it, the signal level would be too low, no matter how superior the antenna might have been due to the nature of the Schuman Resonance. With that knowledge, considerable thought was given when designing an amplifier for the system. There were many types and designs of amplifiers available so careful consideration was required to determine the best design to select. There were two major points that were considered for the amplifier: filtering and the type of amplifier.

The first thing to consider was whether or not filtering was necessary for this application. When analyzing filtering, some things needed to be considered that included the frequency level, 7-15 $\mathrm{Hz}$ and complexity and requirements of a filter at a signal level of $1 \mu \mathrm{V}$. With the frequency range being below $100 \mathrm{~Hz}$, there were additional things to consider. One of those things being the very strong $60 \mathrm{~Hz}$ signal that resonates from all power sources in the United States. This signal could easily knock out any signal that the system received. At such low frequencies, it was very difficult to design narrow bandwidth, conventional analog filters to notch out $60 \mathrm{~Hz}$ and not interfere with the target frequencies.

Hardware filtering was attempted at first. This circuit had several stages involved in the design that included gain stages a $60 \mathrm{~Hz}$ notch filter and a low pass filter to help filter out higher frequencies. Figure 1 shows a high-level overview of the circuit. 


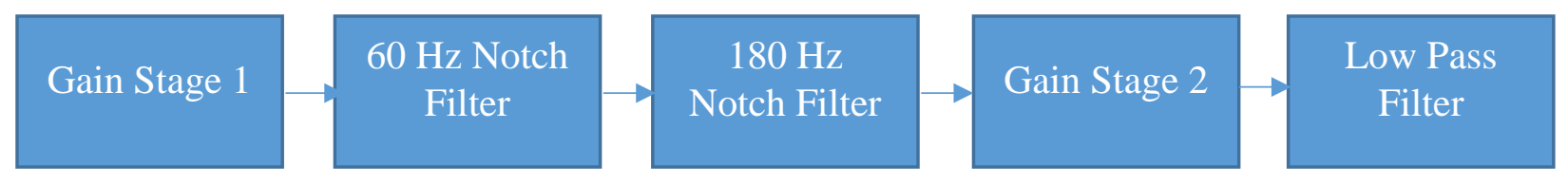

Figure 1 - Hardware filtering and amplifier attempt overview

The goal with this design was to amplify the signal first and then notch out unwanted signals, like 60 and $180 \mathrm{~Hz}$, and then pass through another gain stage before the final low filter was applied. The circuit diagram is shown below.

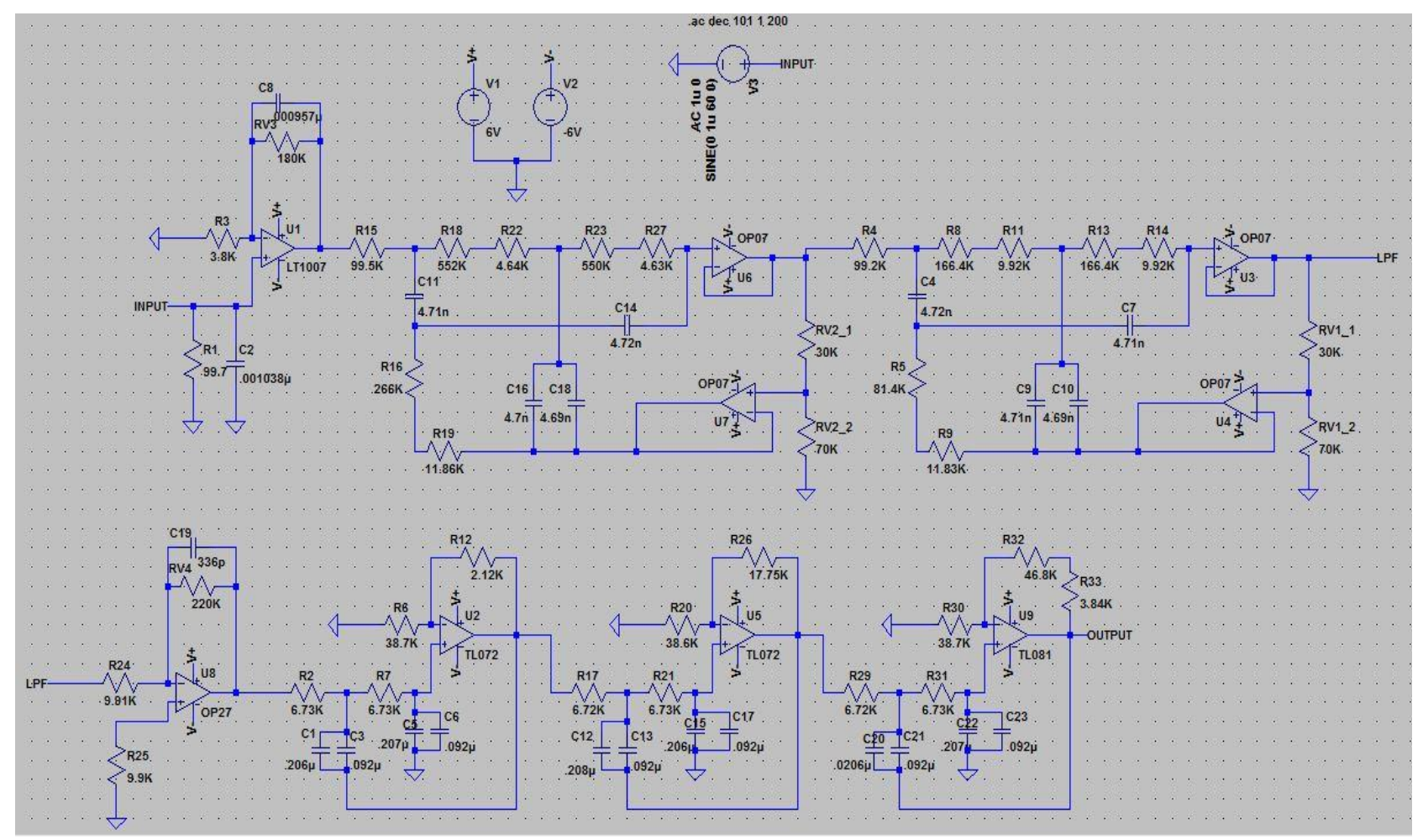

Figure 2 - Hardware filtering and amplifier schematic

The circuit was then tested using LTSpice for the simulation and then was constructed using a prototyping board and further testing was performed. The circuit was tested using a $1.5 \mu \mathrm{V}$ signal that was input into the circuit and the output was measured. The input and output were then compared to each other to determine the overall gain of the system. The result of that test is shown below. 


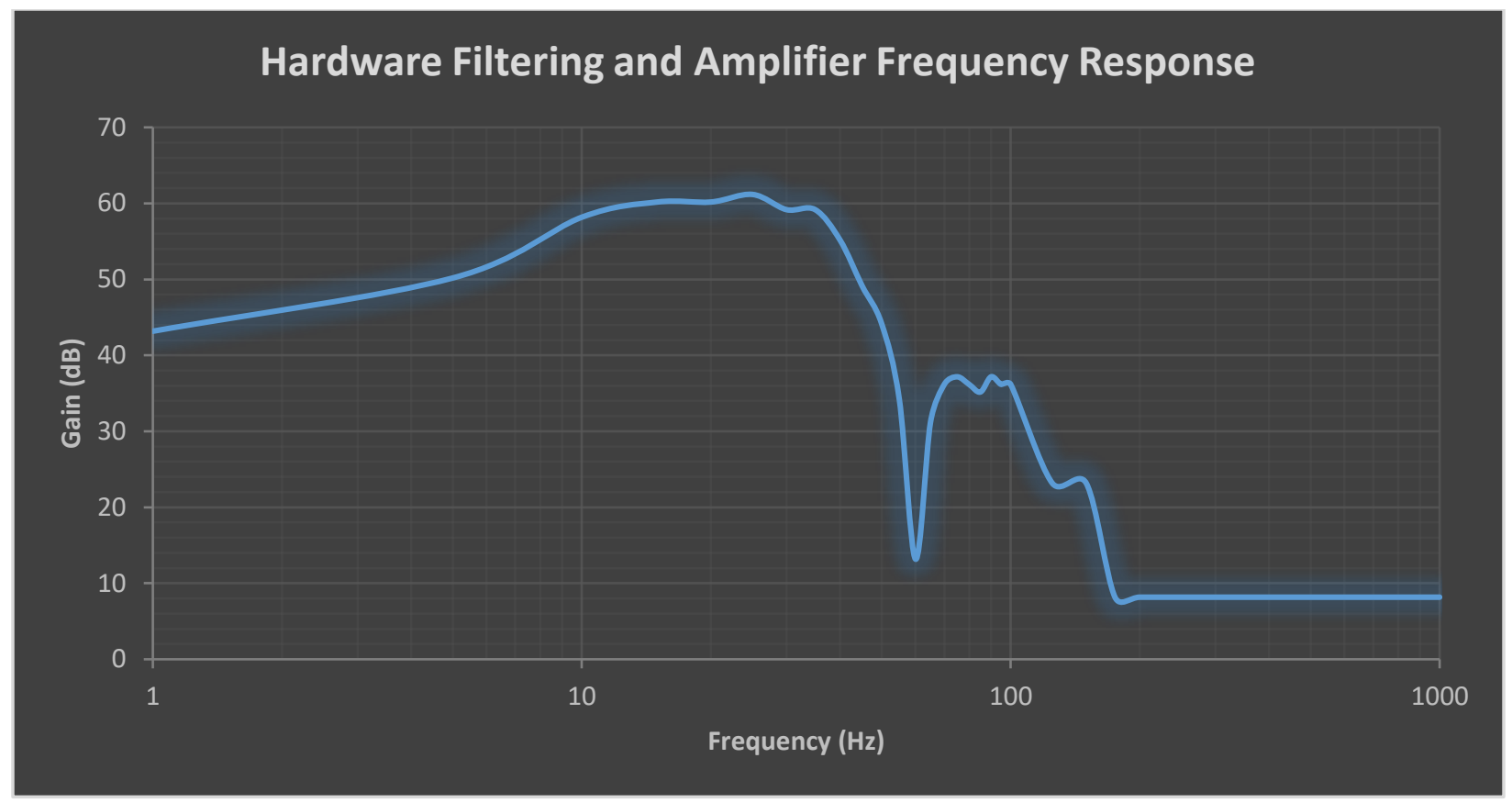

Figure 3 - Hardware filtering and amplifier frequency response

From the results, the performance of this amplifier can be seen. The amplifier did not perform well under $10 \mathrm{~Hz}$ which includes part of the target frequency range. Also, the complexity of the circuit brought several challenges when it was constructed due to the number of amplifiers and connections that had to be made. Although this amplifier had considerable gain a higher gain amplifier was needed to be able to amplify signals at the level of the Schuman Resonance. To help reduce the complexity of the circuit the filtering would be removed and would be performed in software, which would also allow for more control of the filters.

The second thing that was considered was the type of amplifier. Two types of amplifiers were considered. The first criteria for the amplifier was the ability to achieve a maximum signal gain with as little noise as possible. Due to the low frequency and low signal level, the system needed to handle DC, or near DC, signals so that the low frequencies of interest could pass without any sort of attenuation. The two types of amplifiers under consideration were instrumentation amplifiers and operational amplifiers. An operational amplifier is a generic gain block that can be configured in multiple ways. An instrumentation amplifier is a much more specific combination of operational amplifiers. In the following section, both types will be compared to see if a custom operational amplifier would have been better, or if a standard instrumentation amplifier would have been the correct approach.

An operational amplifier, more commonly known as an op-amp, is a basic, general purpose gain block that can be configured in several ways to achieve a desirable circuit [10]. Since the op-amp was so configurable, a custom version of an instrumentation amplifier could have been designed using multiple operational amplifiers. The figure below shows a typical differential operational amplifier. Differential amplifiers help reduce common mode noise between the two inputs and could have been used in this situation. 


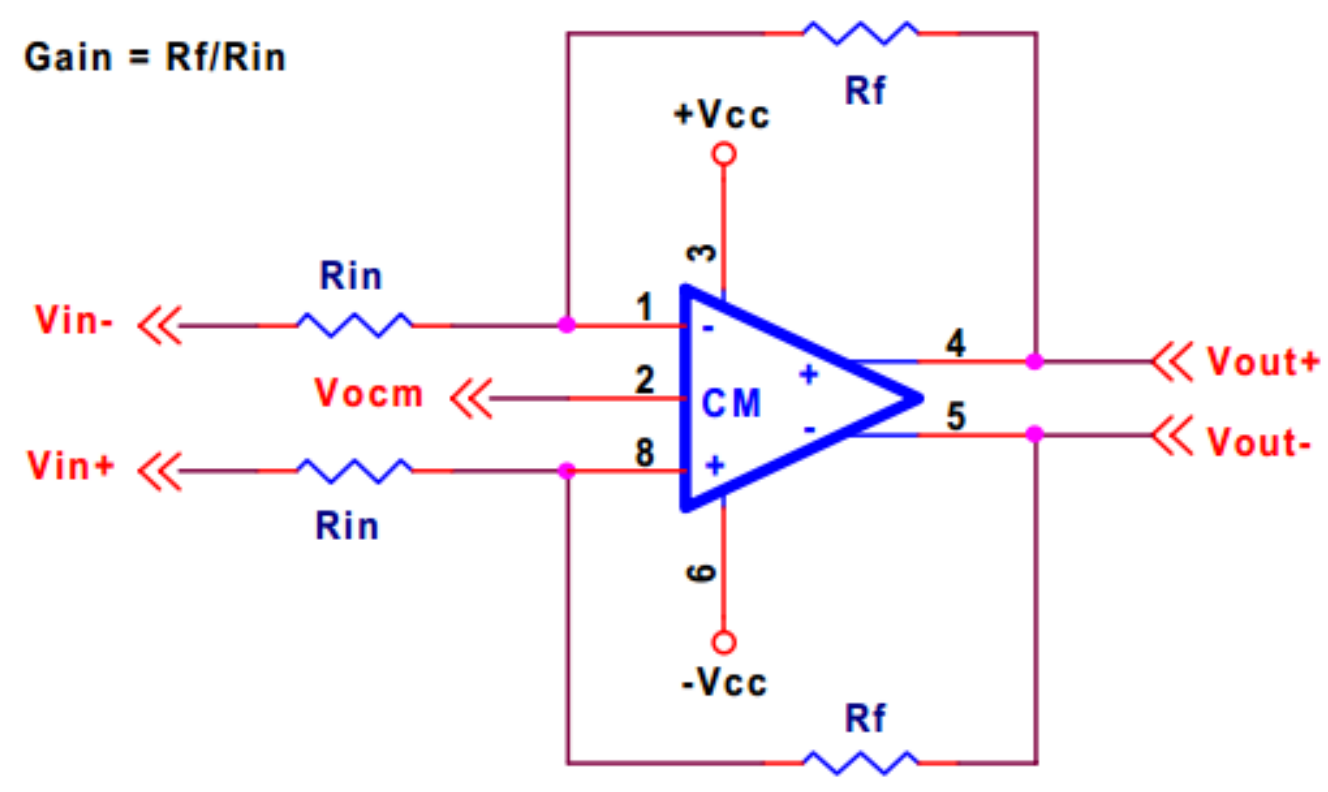

\section{Figure 4 - Typical Operational Amplifier Circuit [11]}

The above figure was just one example of the many possible circuit configurations that could have been used. The gain for a configuration like this is very simple and is shown below.

$$
\text { Gain }=\frac{R_{f}}{R_{\text {in }}}
$$

With this simplicity, it was easy to determine what resistors would be needed to achieve the design that was required. When trying to achieve a gain that is large enough to help recover a signal buried in noise, this could be a possible configuration, but the major issue with this design was the amount of noise that was generated by a typical, cost-effective operational amplifier.

The noise that needed eliminated or attenuated was noise that comes from other sources that were no of no interest to the system. These sources include: $50 / 60 \mathrm{~Hz}$ from power lines, radio stations and systems that may use frequencies in the $\mathrm{kHz}$ range. These signals when powerful enough had the ability to swamp out any signal that was of interest. The differential inputs helped eliminate some common mode noise, but most of that noise would have still been amplified and corrupted the signal. To combat that, a design was examined that used three operational amplifiers to create the following circuit. 


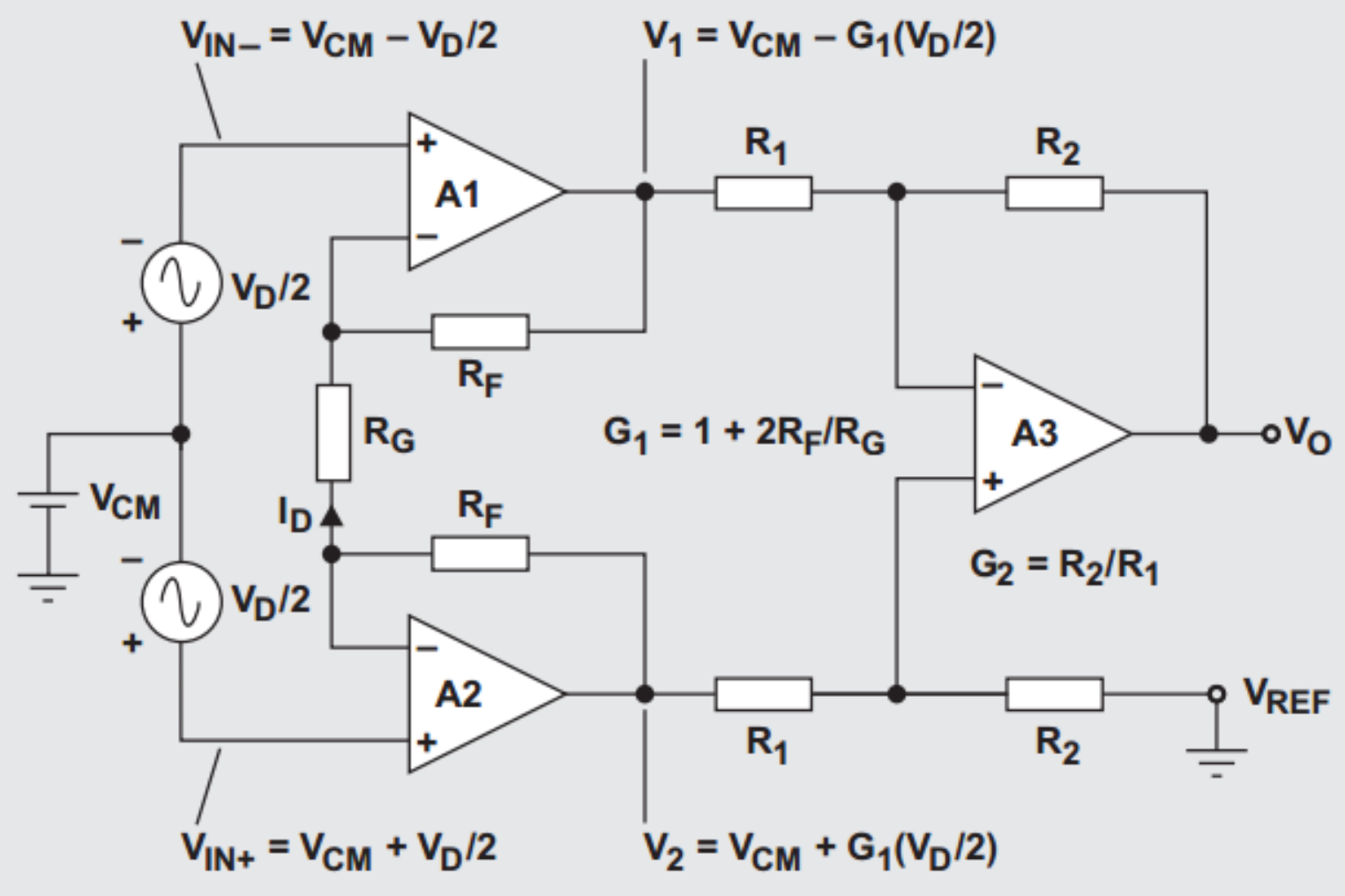

Figure 5 - Operational Amplifiers used to build instrumentation amplifier [12]

The figure above is an instrumentation amplifier which is an amplifier circuit that has a differential input and a single-ended output with respect to a reference terminal [11]. These kinds of amplifiers are exceptionally good at removing common mode noise between two differential inputs, which was the major reason to consider it. The hope for this circuit was that common mode noise between the two ends of the loop antenna would be removed, and the signal would have been much cleaner. This could then be fed as a single ended signal into the analog to digital converter, A/D, to get a more accurate signal. The figure below shows an implementation of the instrumentation amplifier; each triangle represents a typical operational amplifier. 


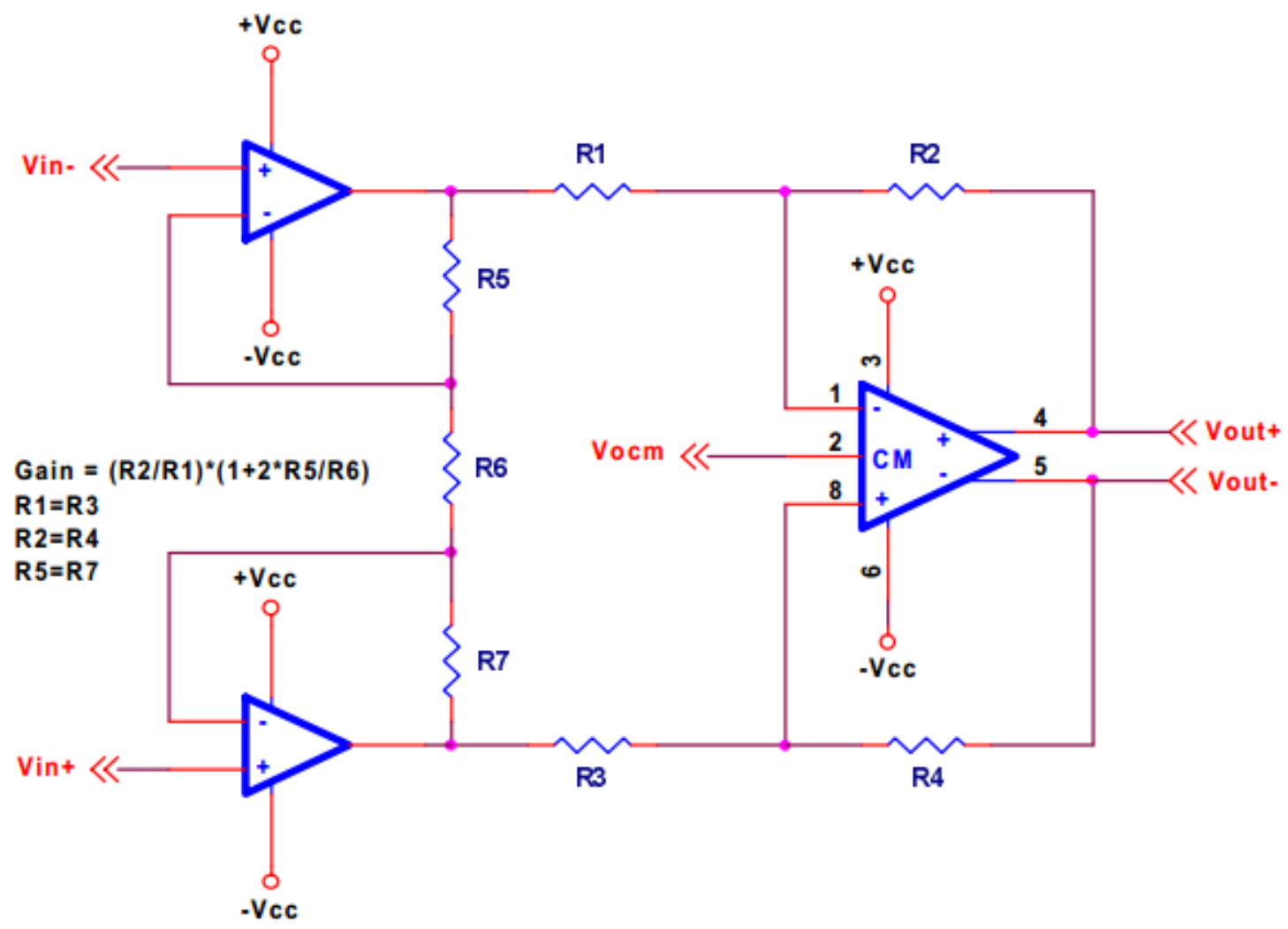

Figure 6 - Typical Instrumentation Amplifier Design [11]

In Figure 3, the instrumentation amplifier is a simple network of operational amplifiers that are configured to be single ended inputs at the input. The circuit uses resistors and a second stage amplifier to eliminate as much common noise as possible while still trying to achieve a large gain. For this circuit, the gain equation is as follows:

$$
\text { Gain }=\left(\frac{R_{2}}{R_{1}}\right) *\left(1+\frac{2 R_{5}}{R_{6}}\right)
$$

This equation was used to determine the resistors needed to achieve the necessary gain.

The big difference between the instrumentation amplifier and the operational amplifier was their level of configuration and ability to change their functionality. Instrumentation amplifiers were very restricted in what could be changed. The only thing that could be configured was the gain and, in some cases, the frequency range. In operational amplifiers, almost everything was configurable and could be customized to whatever the user/designer needed it to be [10]. When considering which amplifier to use if only one of each amplifier was to be used, then the instrumentation amplifier would typically give better noise performance than a single op-amp, although the same instrumentation amplifier could have been designed with multiple op-amps. To also help reduce the complexity of the hardware only one amplifier was to be used, further reducing the previous hardware filtering attempt to only one gain stage. 
With only one amplifier to be used selection of the amplifier to use was crucial Various operation amplifiers and instrumentation amplifiers were considered to be used in the amplifier including all the amplifiers that were used in the initial hardware filtering attempt. The list was not an exhaustive but includes some the more popular amplifiers that are used in low frequency systems. The list of amplifiers includes the following amplifiers: TL072, TL081, OP07, LT1007, LT1167. The TL081 [15] and the OP07 [15] did not have very good noise performance so those two removed from the list, leaving only the TL072, LT1007 and LT1167. All three remaining amplifiers had very comparable noise performances at low frequencies, but with the antenna an instrumentation amplifier would be more useful so that the common mode noise could be removed and so that both antenna ends could be connected the amplifier directly. This narrows the list down to only the LT1167 as it was the only amplifier under consideration that was available as an instrumentation amplifier [16] [17] [18].

\subsubsection{Amplifier components and final design}

Using the formulas and calculations from the instrumentation amplifier was chosen as the final amplifier design. The circuit used is shown below.

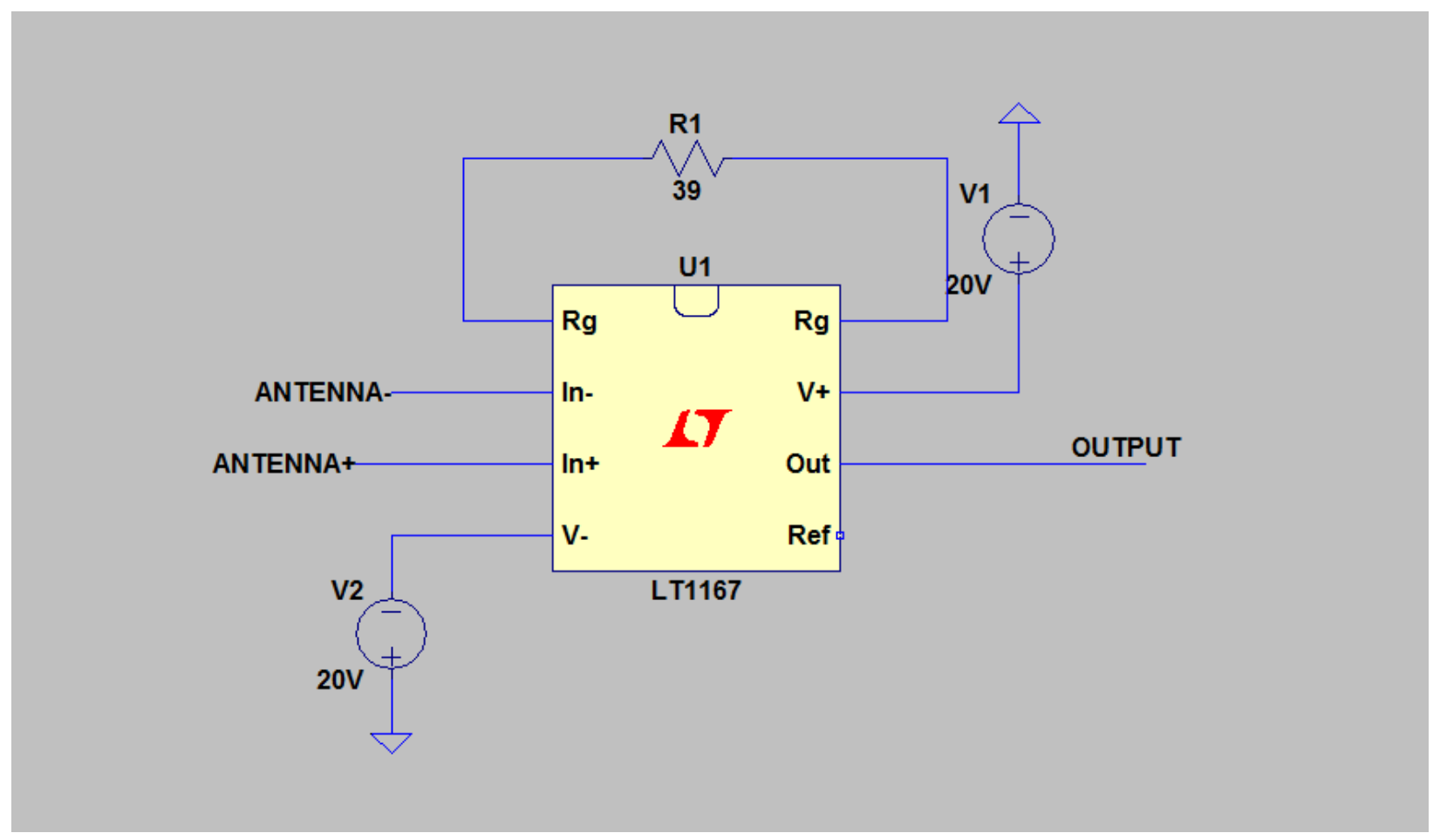

Figure 7 - Final Amplifier Design in LTSpice

The selected design was based on the instrumentation amplifier design that was mentioned above. The figure below shows the internal circuitry of the instrumentation amplifier that was selected. 


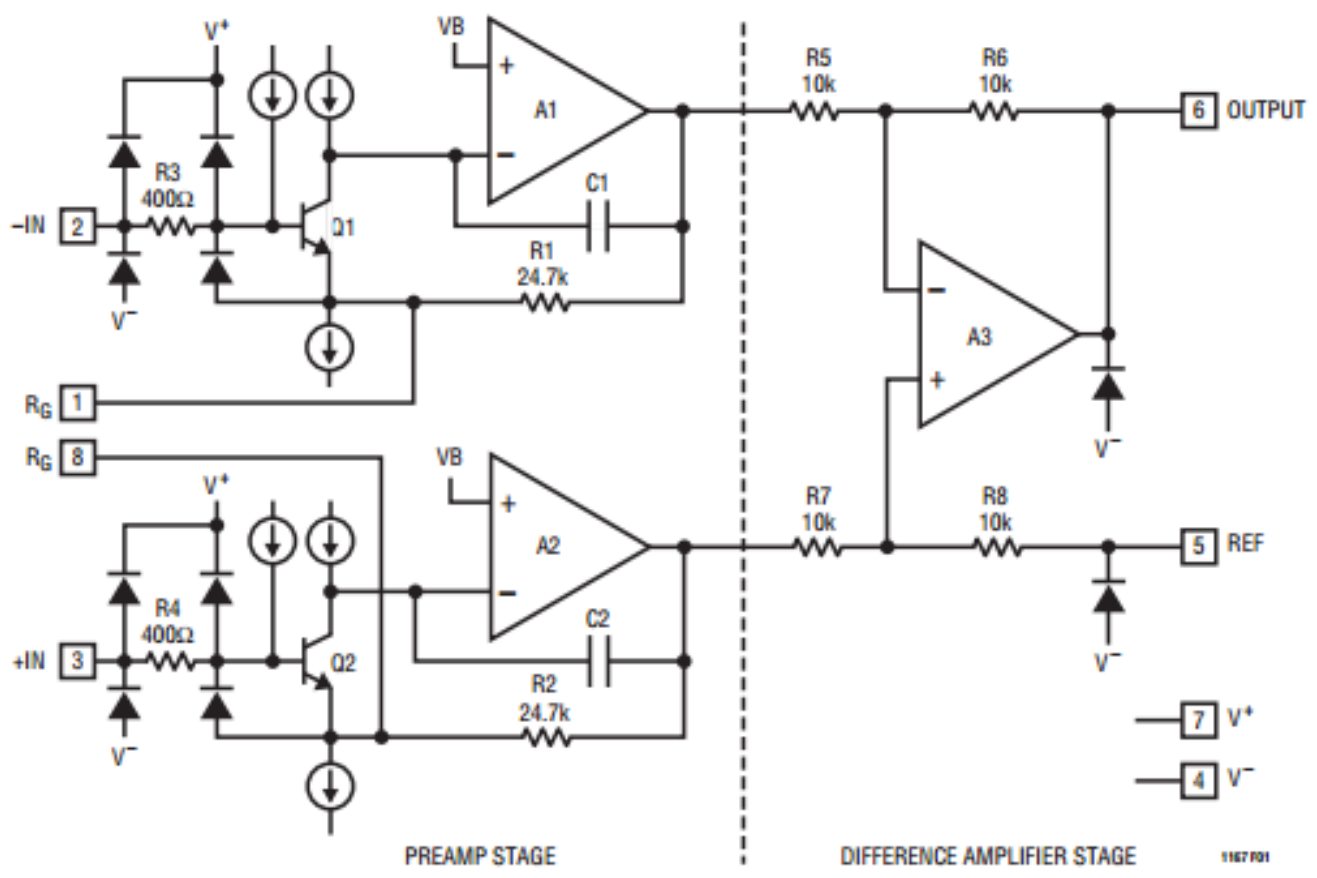

Figure 8 - LT1167 Internal Configuration [14]

The instrumentation amplifier that was chosen is the Linear Technology 1167 or LT1167, which is a single resistor gain programmable, precision instrumentation amplifier [12]. This type of instrumentation amplifier was chosen due to its low generated noise at low frequencies and its gain potential. It can be seen how the noise affects the amplifier and its output signal by looking at the following figures from the datasheet.

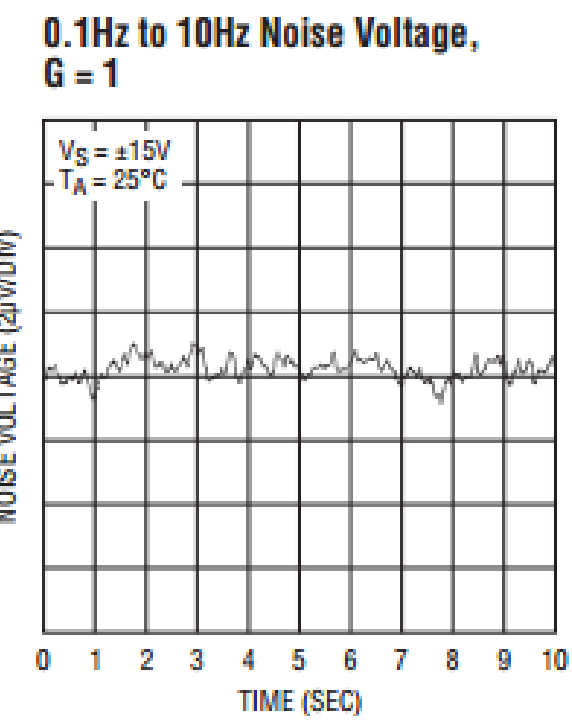

\section{$0.1 \mathrm{~Hz}$ to $10 \mathrm{~Hz}$ Noise Voltage, Referred to Input, $\mathbf{G}=\mathbf{1 0 0 0}$}

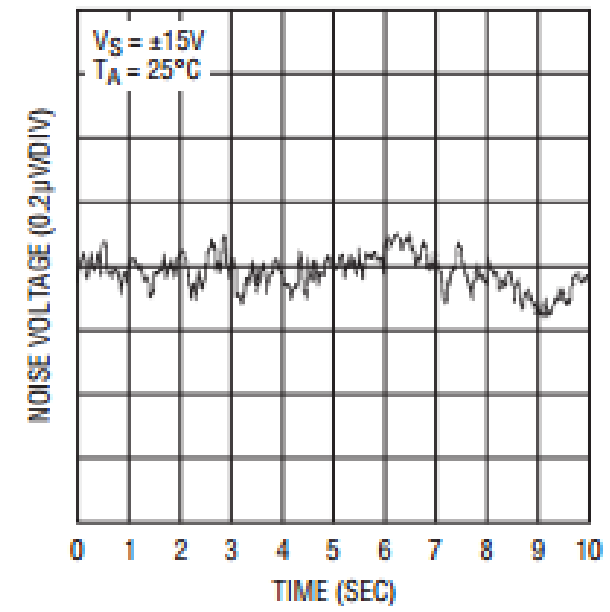

Figure 9 - Noise characteristics for LT1167 [14] 
Also with this amplifier, it could do some filtering on its own due to the roll off capacitors inside the amplifier itself. Below is the specification image showing where that roll off occurred.

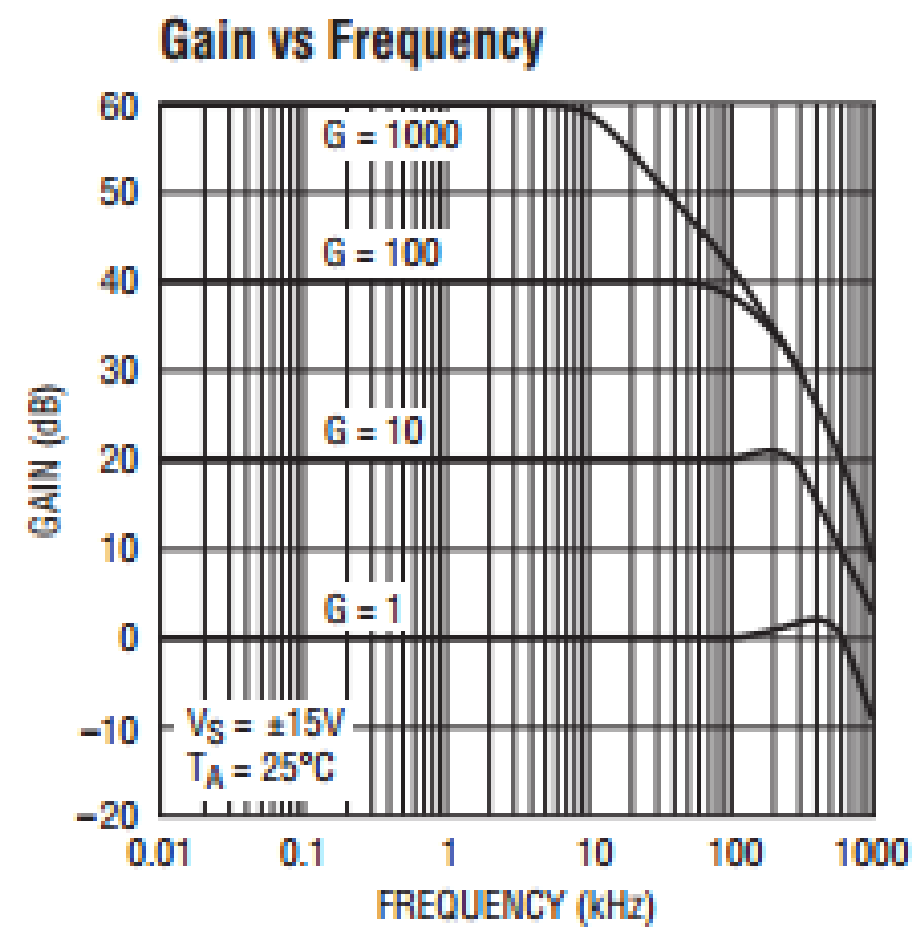

Figure 10 - Theoretical gain for LT1167 [14]

From the graph, the roll off began at around $10 \mathrm{kHz}$ with a gain of $60 \mathrm{~dB}$. The gain for the circuit was close to that, so it was expected that a roll off would be around this point when the amplifier was tested. The gain of the amplifier was determined by using the gain formula that was pulled from the documentation for the amplifier which was,

$$
G=\left(\frac{49.4 k \Omega}{R_{G}}+1\right)
$$

If,

$$
R_{G}=39 \Omega
$$

Then substituting in, the gain comes to be 1,268 . That gain equation, however, only gave the output in standard units and the gain needed to be in $\mathrm{dB}$ to make an accurate comparison to the graph. To do so, the gain was converted into $\mathrm{dB}$ using the following equation. 


$$
G_{d B}=20 * \log (G)
$$

Which after substituting gave a final formula for the gain,

$$
G_{d B}=20 * \log \left(\frac{49.4 k \Omega}{R_{G}}+1\right)
$$

If,

$$
R_{G}=39 \Omega
$$

Then substituting the values in the gain came out to be approximately $62 \mathrm{~dB}$. From this value, it was assumed that a significant roll off, as shown in Figure 7, would be achieved at the $10 \mathrm{kHz}$ mark. This roll off proved to be helpful in the system as it allowed the system to filter out higher frequency signals that were very strong and would have been picked up by the antenna and swamped the low-frequency signal.

With this design, it was expected to be able to receive the signal through inputs of the amplifier and amplify them on the order of $60 \mathrm{~dB}$ to send through the rest of the system. The output voltage estimate for the amplifier with a $1 \mu \mathrm{V}$ input was somewhere around $1 \mathrm{mV}$ leaving the amplifier. Such a signal should then be ok for the "line input" of a PC sound card.

\subsection{System Design}

The final design of the system involved combining the antenna and the amplifier. Then finding a way to visualize the data so it could be useful. To do this, several things needed to be considered.

The first was how the antenna and amplifier would interface together. Since the antenna was a loop, it was known that it had two terminals which were connected to the positive and negative inputs of the amplifier. This type of connection connects well with the instrumentation amplifier. The amplifier would help in removing any common noise that would be present on both terminals of the antenna, one such signal was the man made $60 \mathrm{~Hz}$ signal that was present everywhere due to the United States power system. BNC connectors and cables were used to physically wire the two items together. The antenna would have a BNC connector and the amplifier would also have a BNC connector and the two would be connected via a BNC cable. Below is a high-level overview of how the amplifier would connect with the antenna. 


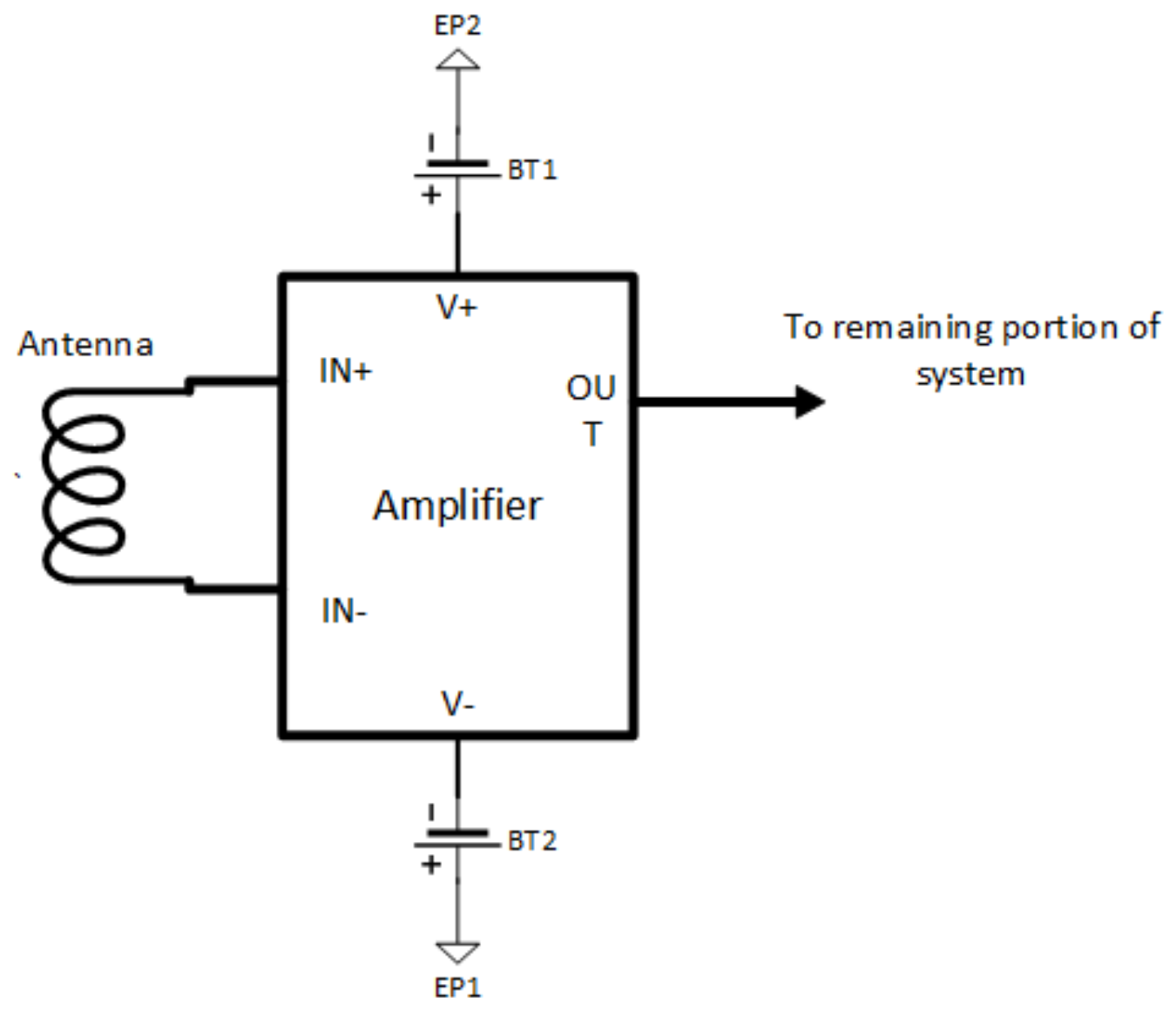

Figure 11 - Block diagram of system design

The next step was to determine how that signal would be processed and visualized for analysis and usage. There were many things to consider here. The first choice was to simply view the output on an oscilloscope and watch the signal level change. The oscilloscope in question is shown below.

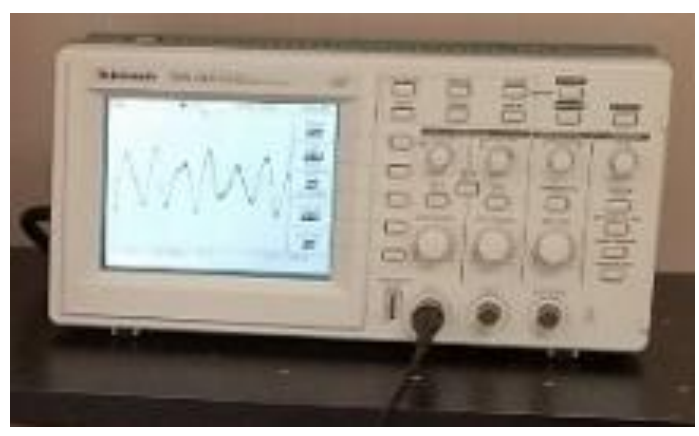

Figure 12 - Oscilloscope used for testing

The main drawback to this approach was that the oscilloscope that was available for the system was not able to save data over time, so other than getting that raw data at the instance there was not much other processing and analysis that could be performed. The second option is very similar 
but can make use of the power of modern computers. That option was a digital oscilloscope. The one that was under consideration for the system is shown below.

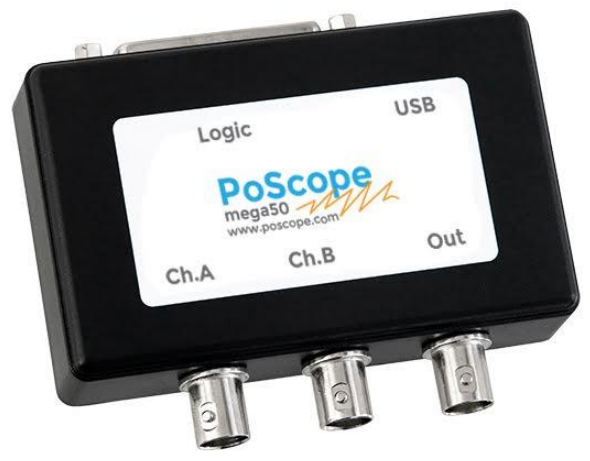

\section{Figure 13 - Digital oscilloscope [13]}

This digital oscilloscope was called the PoScope which allowed the user to run a piece of software and store, process, and visualize that data just like a traditional oscilloscope, but with greater control and the ability to create recordings for further processing. The PoScope also had programmable sample rates and quick control of frequency and voltage settings. With this option, there was still no real-time way of watching the data change over time.

The last option, and ultimately the best option, for this application, was to use a typical PC sound card and couple that with software on the computer to act similarly to the digital oscilloscope but with more software features. The sound card used was like the one seen below.

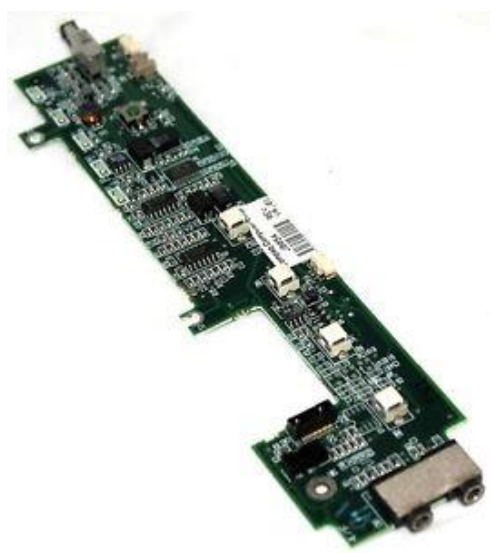

Figure 14 - Sound card from a PC [14] 
The software used with the sound card was a program call SpectrumLab which was free and available to all. [15] The software could show the frequencies as a function, commonly called a waterfall display. This type of software allowed the capture of the data and included storage of the data. It could then be used for further processing if needed. It also helped give an immediate visual to changing frequencies that were captured by the system.

For completeness and to capture as much data as possible, all options were used. The figure below shows the entire setup as a block diagram.

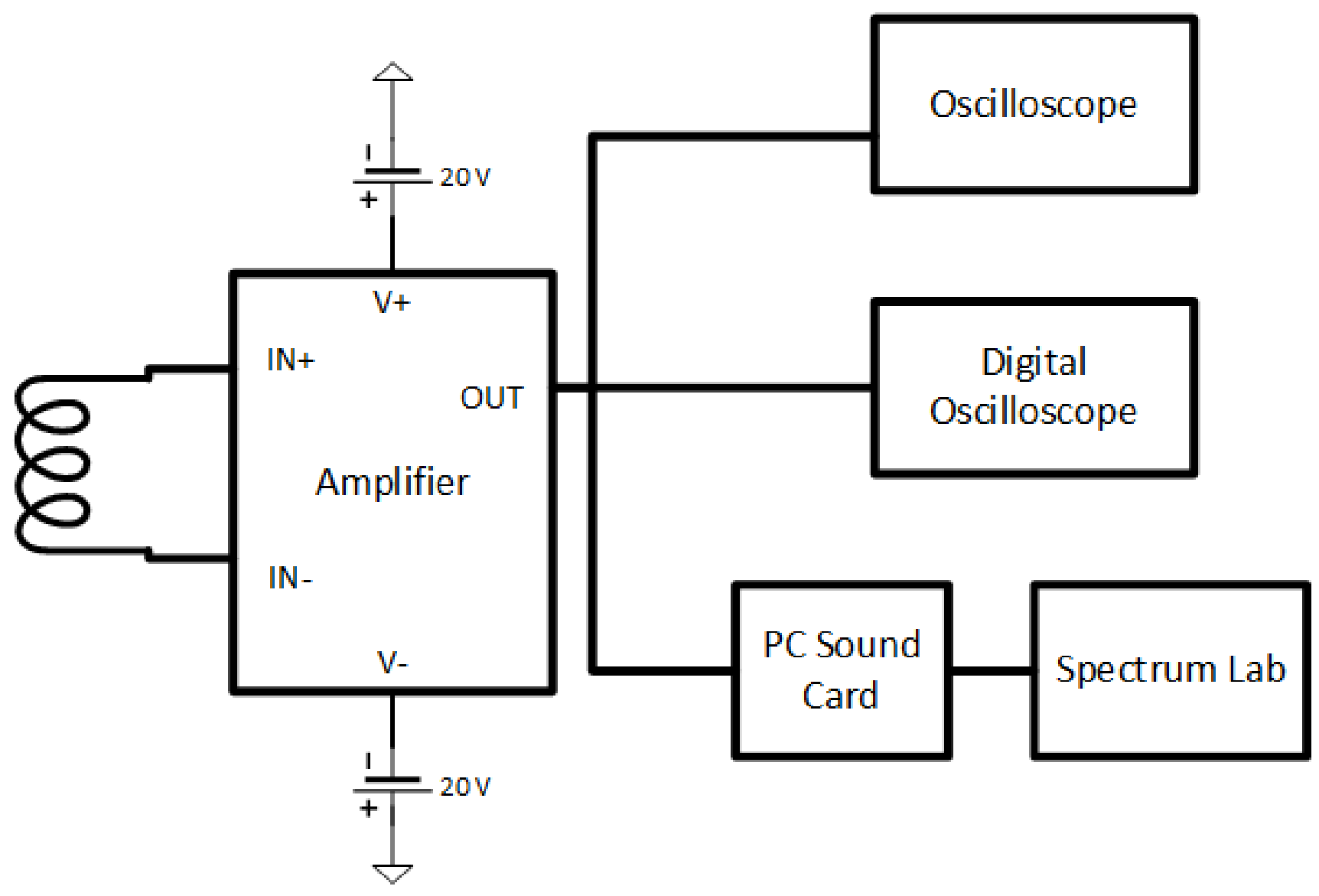

Figure 15 - Full system design

The following chapter discusses the simulation of the amplifier and characterization of the antenna. There was no available simulation to run for the system, the antenna and amplifier together. 


\section{CHAPTER 3: SIMULATION OF AMPLIFIER AND ANTENNA}

\subsection{Simulation of Amplifier}

\subsubsection{Overview}

This section shows the simulation results for the amplifier. The simulation was performed to test the design that was decided upon in the previous section. The simulation could provide valuable information as to how the amplifier worked, how it was able to handle a simulated signal, and how the gain and noise was handled with the amplifier.

LTSpice was the simulation program that was chosen to simulate the circuit since the software was developed by Linear Technology and the instrumentation amplifier used was also developed by Linear Technology. By using this software, the exact model for the instrumentation amplifier was used to get a more realistic model of the amplifier.

\subsubsection{Setup}

The circuit components for the amplifier design were added in LTSpice. The following image shows the circuit used for the simulation.

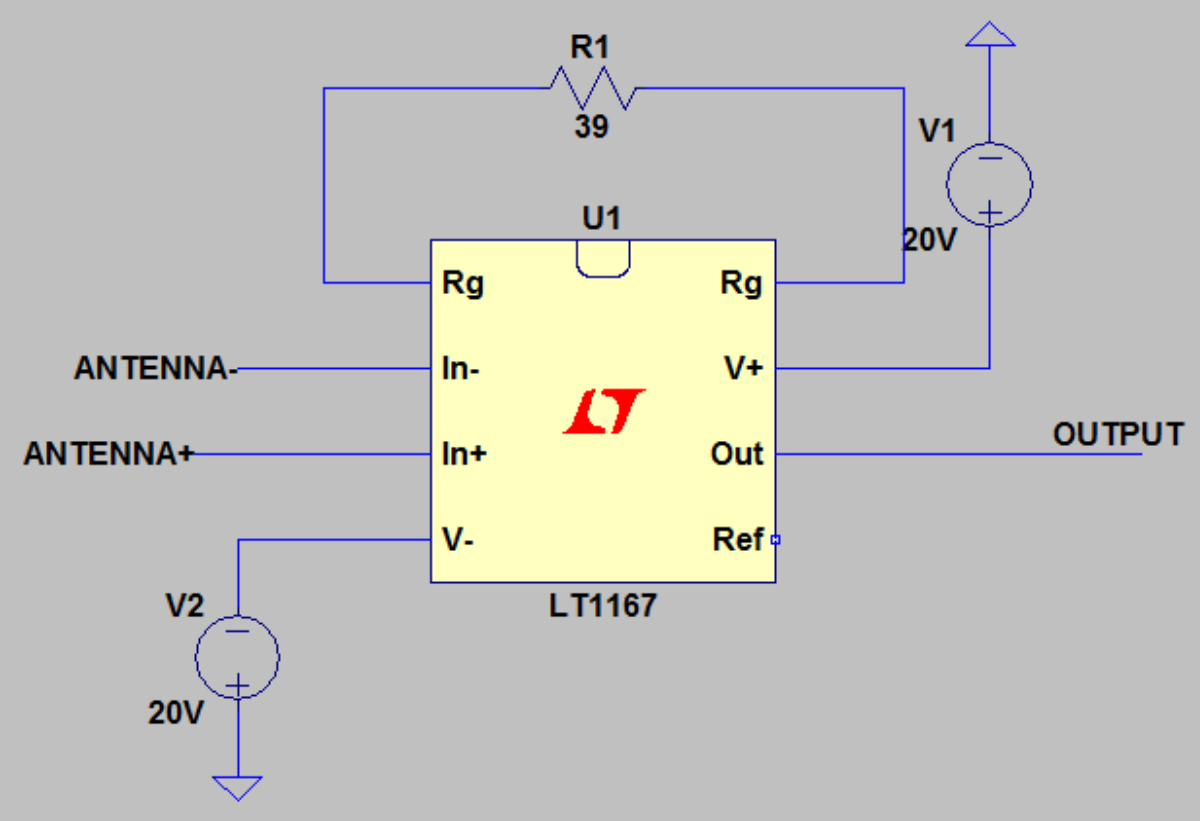

Figure 16 - Amplifier design 
Since the above schematic had the inputs marked as coming from the antenna and LTSpice cannot handle antenna simulations or anything of that nature, a simple sinusoid voltage source was used in its place to simulate the signal that would be received from the antenna. The figure below shows the updated circuit configuration.

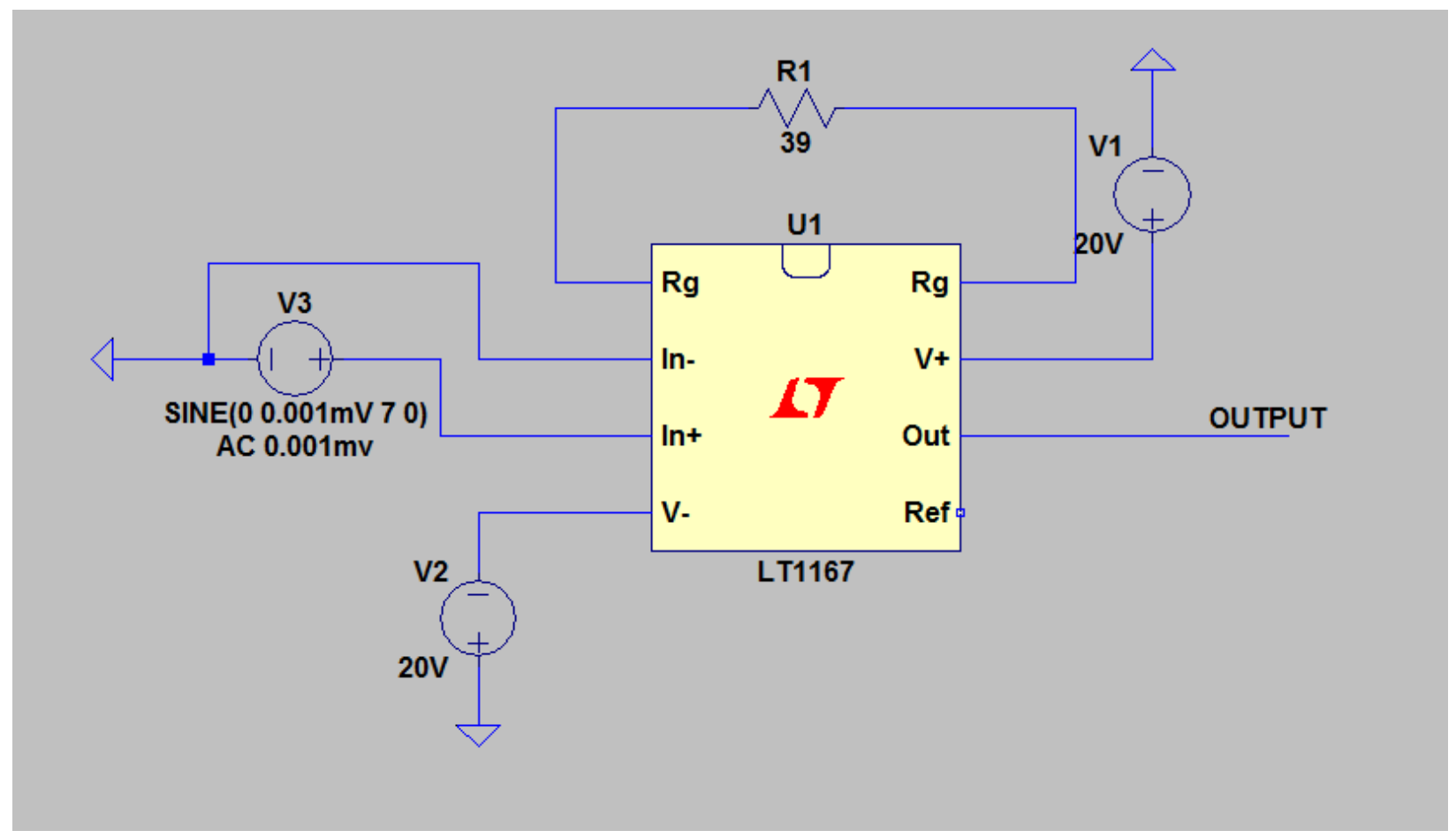

\section{Figure 17 - Amplifier setup for simulation}

Although this configuration was not ideal, two simulations were performed to determine the characteristics of the circuit. The first set of simulation results were the small signal analysis in which a signal very much like the target signal that should be received by the amplifier was used as input into the system. The second set of simulation results analyzed the circuit over the frequency range $0.1 \mathrm{~Hz}$ to $10 \mathrm{kHz}$ which was a range much larger than the target range for the system, so it gave a good analysis of the expected frequency response of the system. The signal voltage fed into the amplifier was of same as the small signal analysis but was swept across all frequencies in that range in specified intervals.

\subsubsection{Small Signal Analysis}

The estimated signal level for the Schuman resonance was approximately $0.0005-0.001 \mu \mathrm{T}$ which was roughly equivalent to $0.158 \mu \mathrm{V}$ [16]. Due to problems with the simulation software, the input value had to be increased to $1 \mu \mathrm{V}$ or the software would crash. This value was used as the amplitude of the sinusoid used in place of the antenna, assuming there was no antenna gain (or loss). The input signal over time (1s) is shown below. 


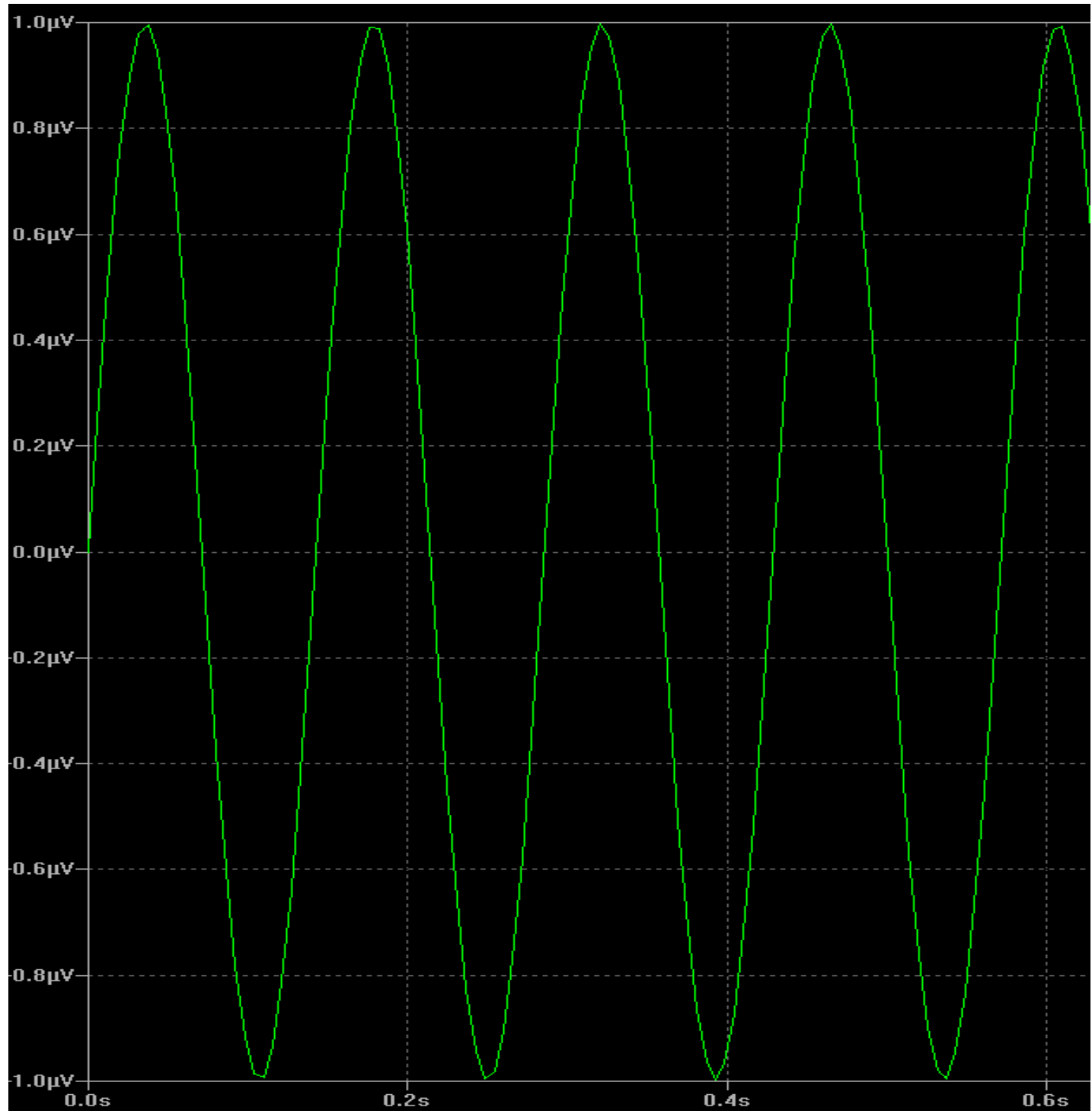

Figure 18 - Input signal for simulation

The peak to peak voltage of the input signal can be seen in the graph as approximately $2 \mathrm{uV}$ and from the amplifier design, the gain of expected output was determined using the gain equation for the amplifier.

$$
G=\left(\frac{49.4 k \Omega}{R_{G}}+1\right)
$$

Where,

$$
R_{G}=39 \Omega
$$


Substituting $\mathrm{R}_{\mathrm{G}}$ in the equation showed that the gain should be 1267.7 approximately. From that, the expected signal level was determined using the following equation.

$$
V_{\text {out }}=G * V_{\text {in }}
$$

Using the equation above, the output voltage should have had a peak to peak signal level of about $2.6 \mathrm{mV}$. Using this calculation to compare what was expected and what the simulation showed, the theoretical performance of the amplifier was tested. Below are the results from the simulation.

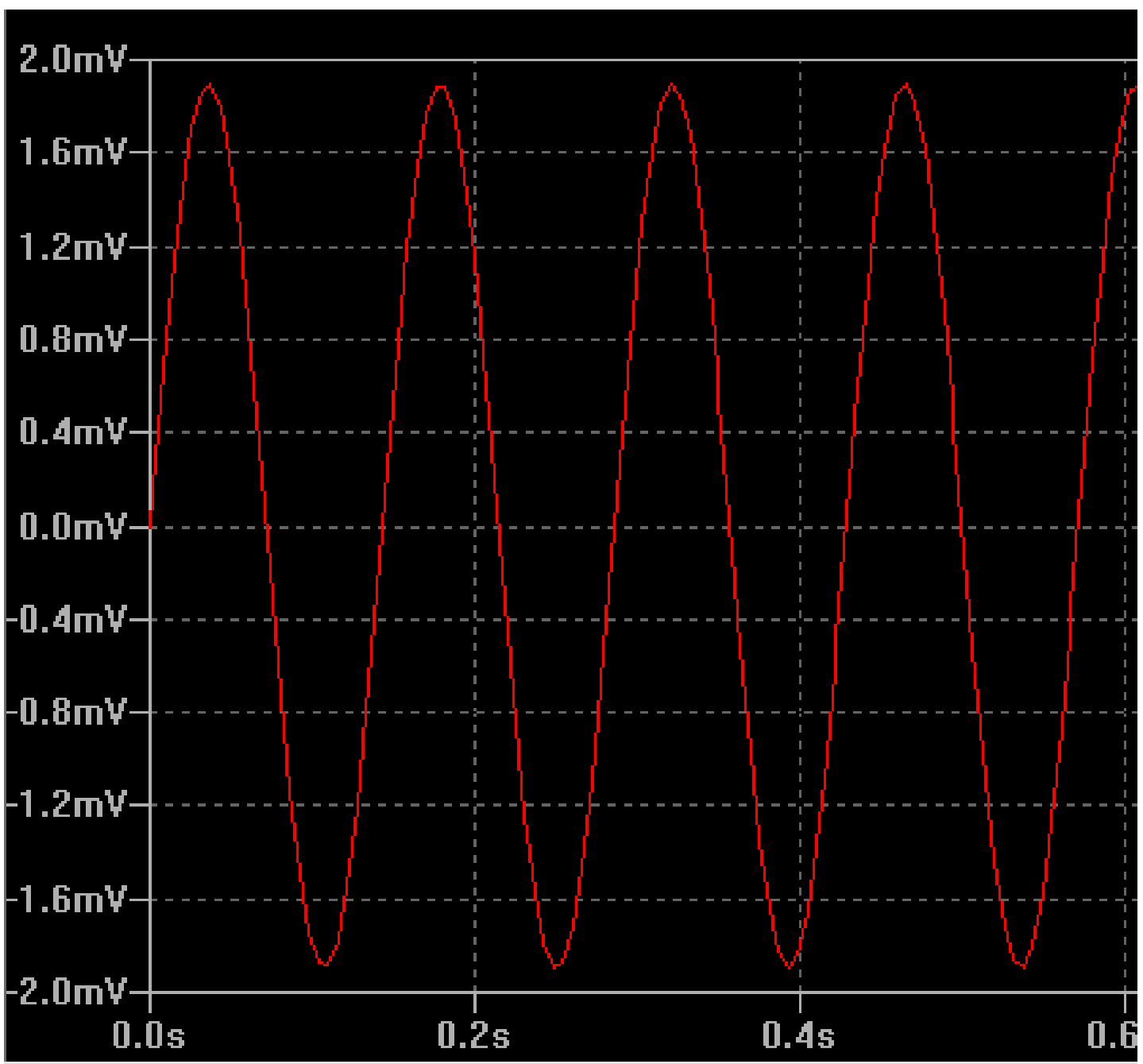

Figure 19 - Output signal from simulation

From this figure, the peak to peak voltage was around $3.2 \mathrm{mV}$ which was a gain that was just under 1600. Comparing the input to the output yielded the graph below. 


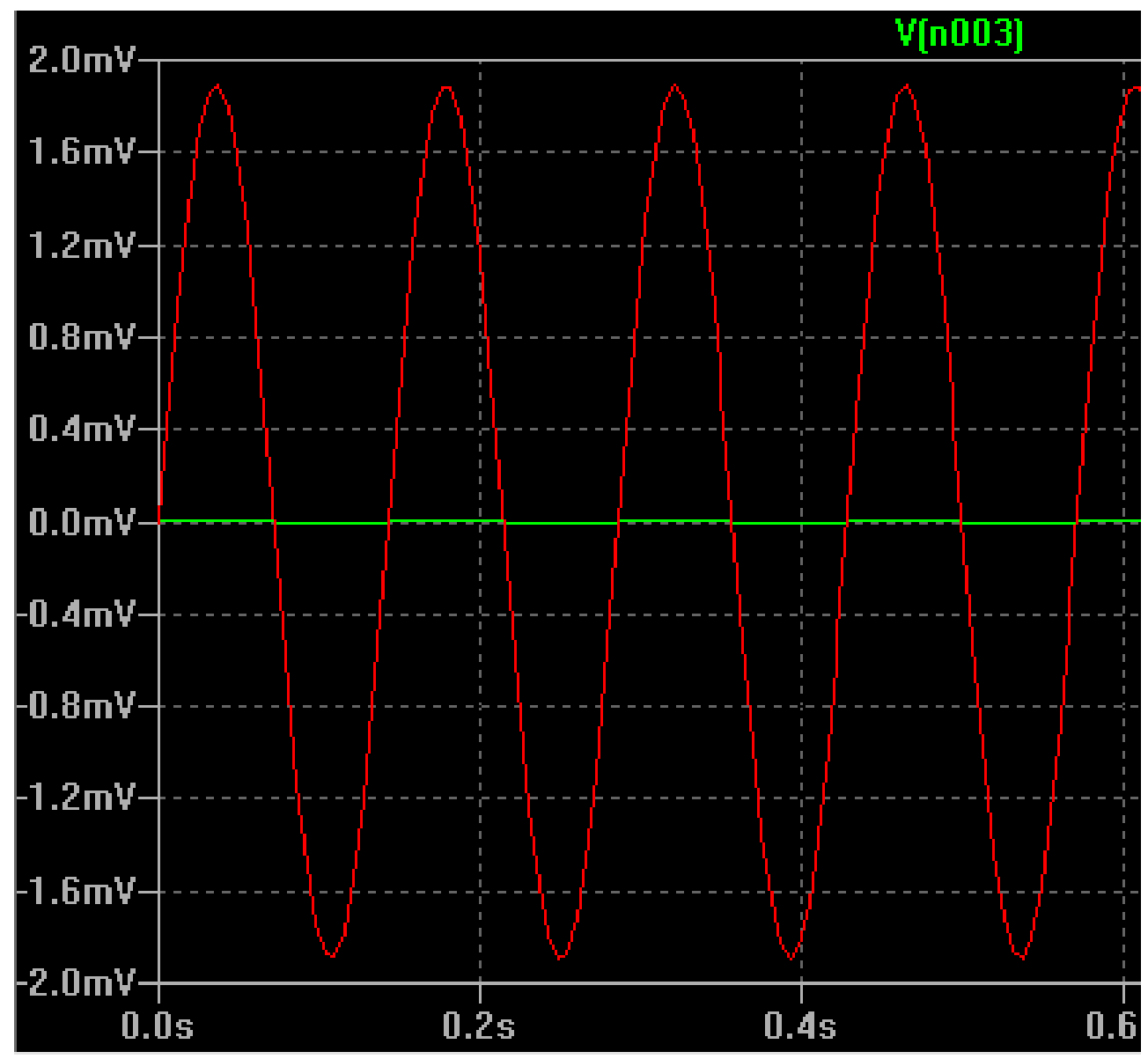

Figure 20-Output (red) signal compared to input (green) signal

This image shows how large the output was compared to the input and how large the gain of 1600 was. From this simulation, the output signal was much greater than the input signal as was expected by the circuit. One thing that was seen was that the gain was greater than what should be expected based on the circuit and instrumentation amplifier. This was due to the instrumentation amplifier type and LTSpice. LTSpice used a specific model for this amplifier type and the additional gain in the system was attributed to LTSpice model issues and not the design itself.

\subsubsection{Frequency Analysis}

For the frequency analysis, the same signal that was previously used ( $1 \mu \mathrm{V}$ sinusoid) was swept across all frequencies from $1 \mathrm{~Hz}$ to $10 \mathrm{kHz}$ on a logarithmic scale. This simulation provided insight into how the system performed at different frequencies. Below is this input signal being used for the simulation. 


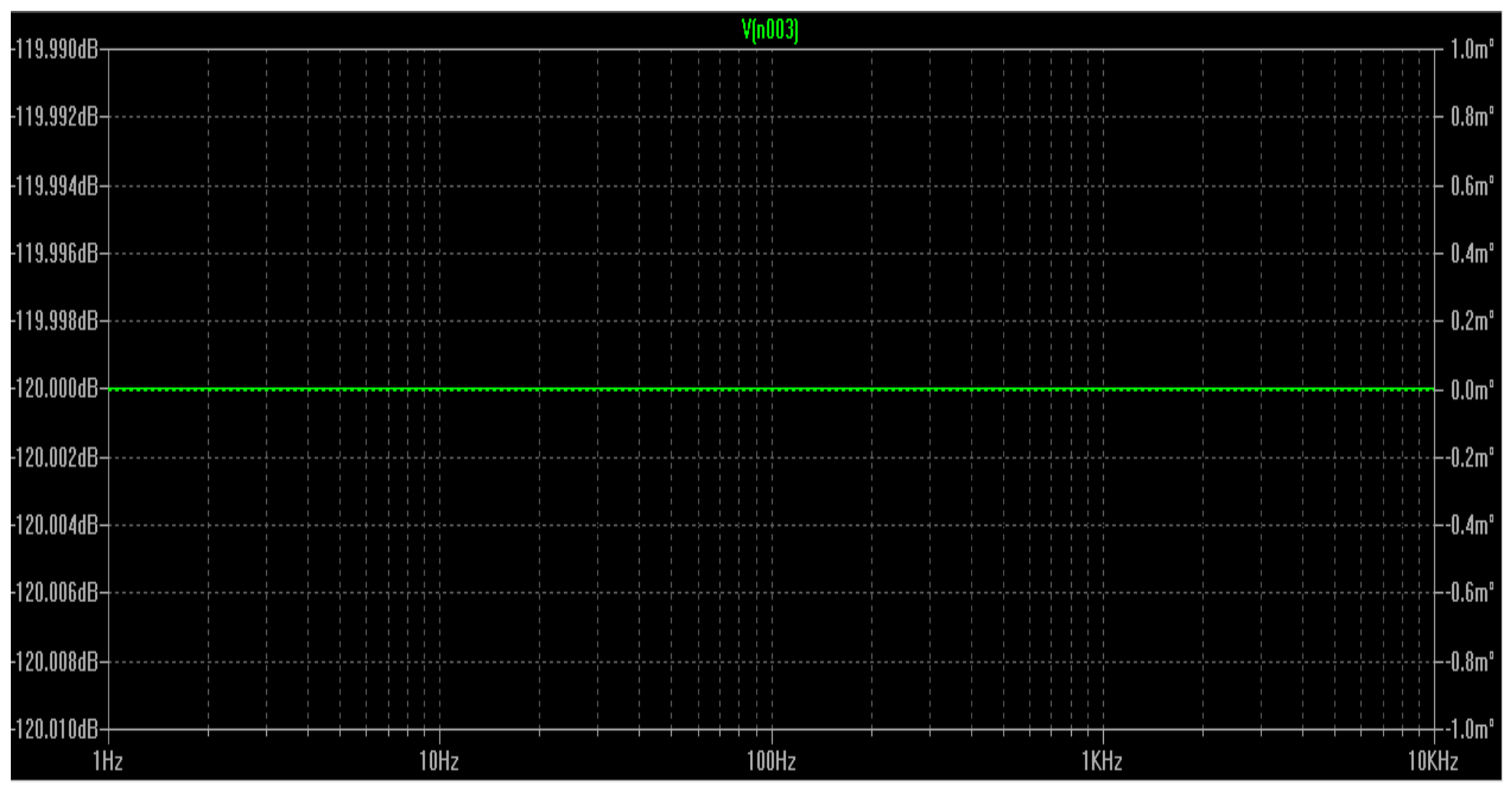

\section{Figure 21 - Input frequency sweep}

The signal level was approximately $-120 \mathrm{~dB}$ or what was roughly equivalent to $1 \mu \mathrm{V}$ which was what the input signal was expected to be. To determine what value the output should be, the gain from above was used and converted to a ratio that was used to determine the correct value in $\mathrm{dB}$. The equation is below.

$$
G_{d B}=20 * \log \left(G_{V}\right)
$$

Where $G_{V}$ is the gain in terms of $V_{\text {out }}$ and $V_{\text {in }}$, so from this the gain was expected to be around 62 $\mathrm{dB}$, which meant that the expected output signal level would somewhere around $-58 \mathrm{~dB}$. Below is the figure showing the output of the circuit. 


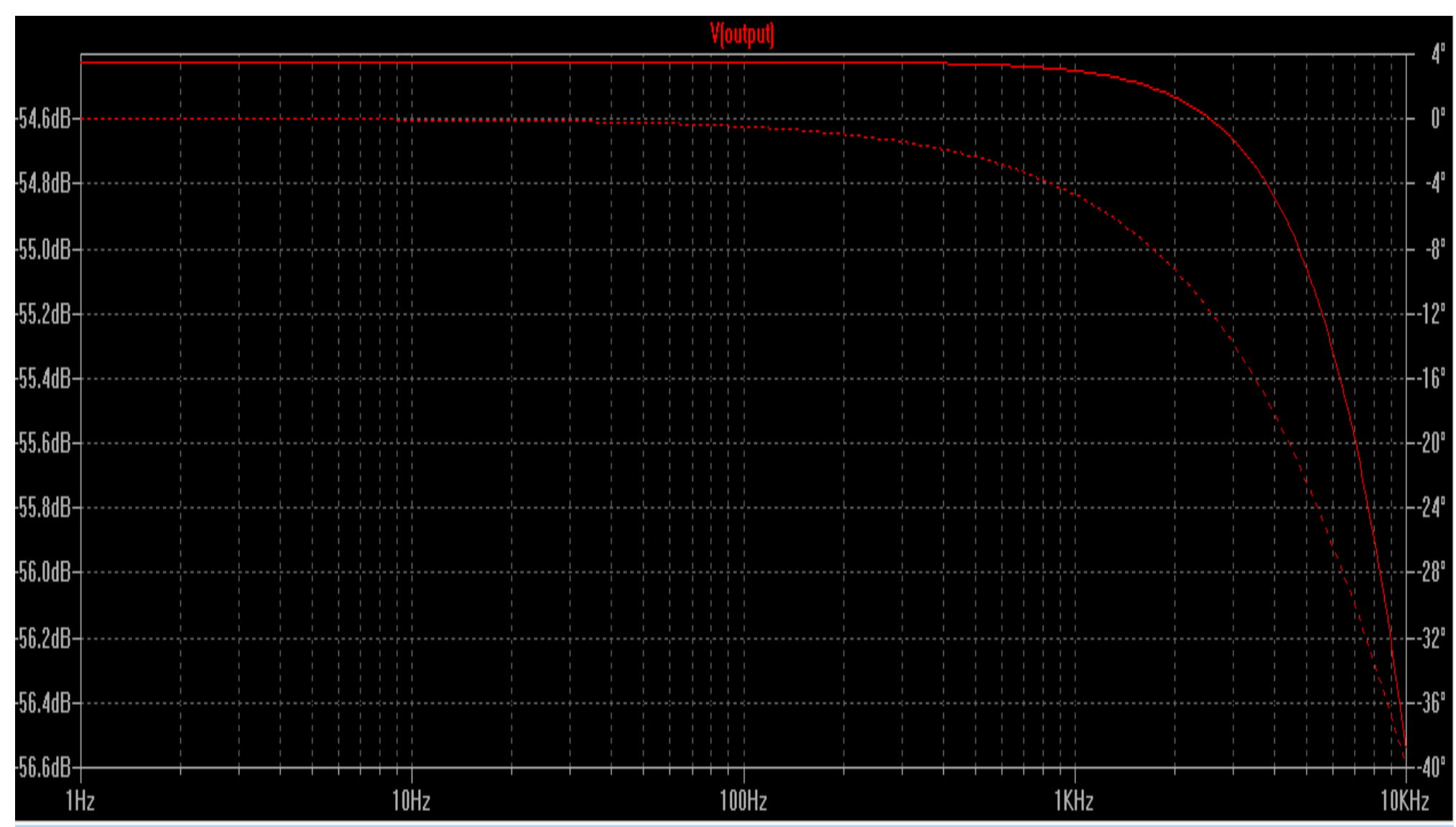

Figure 22 - Output frequency sweep

From this graph, the signal level of the circuit was around $-54 \mathrm{~dB}$ which was close to what was expected to be seen, it was slightly better performance than expected. The cause of this was believed to be from the simulation software and not from the design itself as all calculations were accurate, the formulas were pulled directly from the specification documents and were known to work for other situations. 


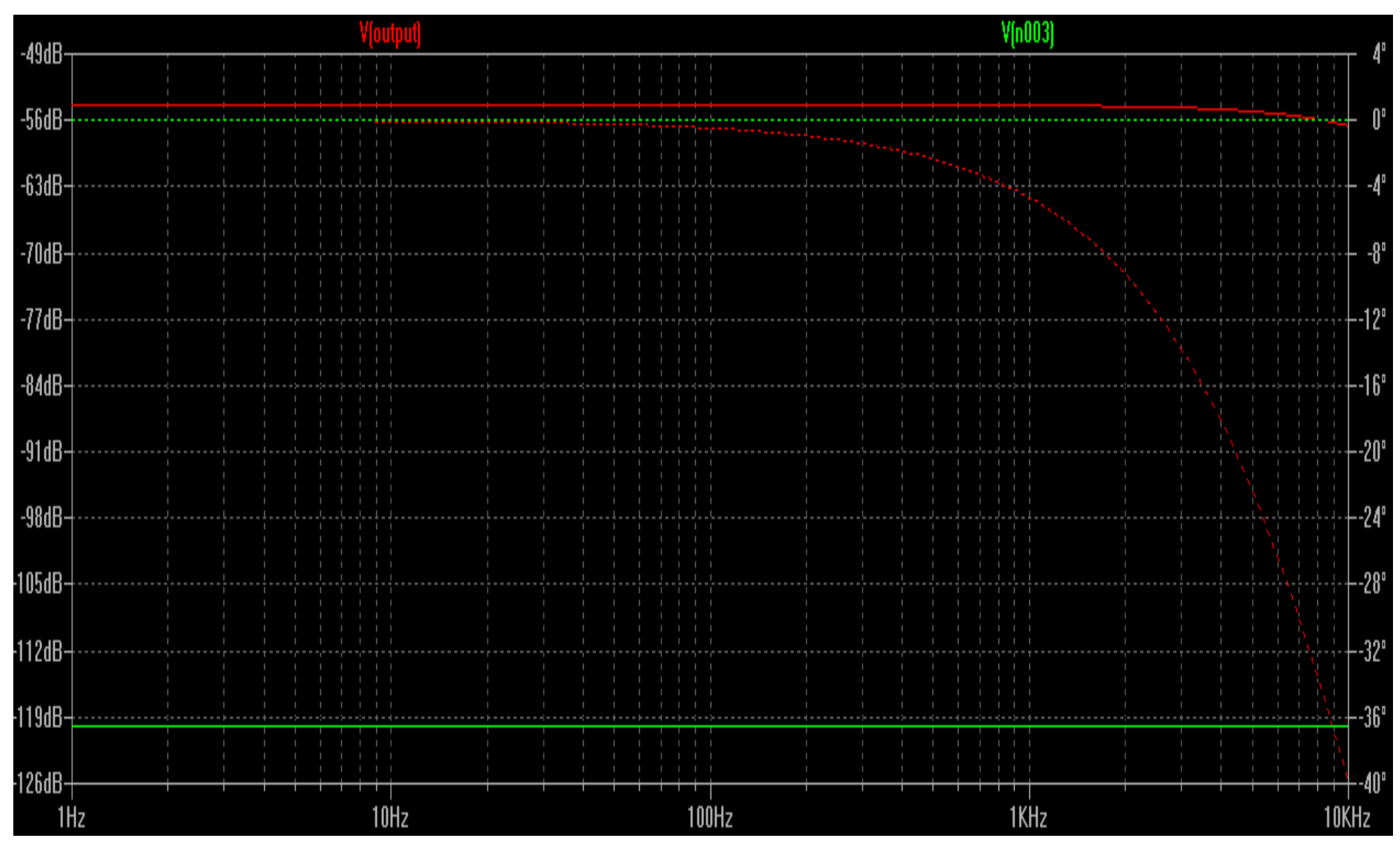

Figure 23 - Output sweep (red) compared to input sweep (green)

This image shows how large the output was compared to the input and how large the gain for the amplifier was. From this simulation, the output signal was much greater than the input signal as was expected by the circuit. This graph also supported the other simulation result because it achieved a gain that was larger than what was expected as well.

\subsection{Characterization of Antenna}

Due to complicated antenna simulation software and difficulty of modeling antennas with software, the antenna was characterized using an LCR meter to determine its impedance at different frequencies so it could be better understood what effect the ferrite core had on the antenna. The LCR meter that was used is the Wayne Kerr 4270. Below are the results from the large antenna that was used on the receiving end of testing. 


\begin{tabular}{rrrrrr} 
& \multicolumn{5}{c}{ Large Antenna } \\
Frequency $(\mathrm{Hz})$ & Inductance $(\mathrm{L})$ & Capacitance $(\mathrm{F})$ & Resistance $(\Omega)$ & Impedance $(\mathrm{Z})$ & Phase $\left(^{\circ}\right)$ \\
0 & none & none & 11.50 & 11.50 & 0.00 \\
50 & 1.5915 & none & 11.50 & 500.00 & 87.00 \\
60 & 1.6534 & none & 11.50 & 623.30 & 87.00 \\
100 & 1.5705 & none & 11.50 & 986.80 & 89.10 \\
120 & 1.5690 & none & 11.50 & 1183.00 & 89.20 \\
200 & 1.5669 & none & 11.50 & 1969.00 & 89.20 \\
300 & 1.5597 & none & 11.50 & 2940.00 & 89.40 \\
400 & 1.5661 & none & 11.50 & 3936.00 & 89.40 \\
500 & 1.5661 & none & 11.50 & 4920.00 & 89.40 \\
600 & 1.5677 & none & 11.50 & 5910.00 & 89.40 \\
700 & 1.5704 & none & 11.50 & 6907.00 & 89.40 \\
800 & 1.5732 & none & 11.50 & 7908.00 & 89.40 \\
900 & 1.5765 & none & 11.50 & 8915.00 & 89.40 \\
1000 & 1.5809 & none & 11.50 & 9933.00 & 89.40 \\
2000 & 1.6608 & none & 11.50 & 20870.00 & 89.10 \\
3000 & 1.8202 & none & 11.50 & 34310.00 & 88.70 \\
4000 & 2.1048 & none & 11.50 & 52900.00 & 88.10 \\
5000 & 5.7296 & none & 11.50 & 180000.00 & 87.00 \\
6000 & 8.2230 & none & 11.50 & 310000.00 & 84.80 \\
7000 & 17.0068 & none & 11.50 & 748000.00 & 76.50 \\
8000 & none & $-2.34 \mathrm{E}-12$ & 11.50 & 17000000.00 & -30.00 \\
\hline 9000 & none & $-3.16 \mathrm{E}-11$ & 11.50 & 570000.00 & -78.60 \\
\hline 10000 & none & $-4.85 \mathrm{E}-11$ & 11.50 & 330000.00 & -84.00 \\
\hline & & & & \\
\hline
\end{tabular}

\section{Table 3 - Large antenna characterization data}

The smaller antenna was primarily for transmitting a test signal that was received by the larger antenna. Below are the results from the small antenna. 


\begin{tabular}{rrrrrr} 
& \multicolumn{5}{c}{ Small Antenna } \\
Frequency $(\mathrm{Hz})$ & Inductance $(\mathrm{L})$ & Capacitance $(\mathrm{F})$ & Resistance $(\Omega)$ & Impedance $(\mathrm{Z})$ & Phase $\left(^{\circ}\right)$ \\
0 & none & none & 1.80 & 1.80 & 0.00 \\
50 & 0.0710 & none & 1.80 & 22.30 & 85.70 \\
60 & 0.0727 & none & 1.80 & 27.40 & 83.50 \\
100 & 0.0705 & none & 1.80 & 44.31 & 87.50 \\
120 & 0.0705 & none & 1.80 & 53.13 & 87.90 \\
200 & 0.0704 & none & 1.80 & 88.41 & 88.50 \\
300 & 0.0702 & none & 1.80 & 132.30 & 88.90 \\
400 & 0.0701 & none & 1.80 & 176.10 & 89.00 \\
500 & 0.0700 & none & 1.80 & 219.80 & 89.00 \\
600 & 0.0699 & none & 1.80 & 263.50 & 89.10 \\
700 & 0.0698 & none & 1.80 & 307.20 & 89.20 \\
800 & 0.0698 & none & 1.80 & 350.90 & 89.20 \\
900 & 0.0698 & none & 1.80 & 394.60 & 89.20 \\
1000 & 0.0698 & none & 1.80 & 438.30 & 89.30 \\
2000 & 0.0698 & none & 1.80 & 877.20 & 89.30 \\
3000 & 0.0702 & none & 1.80 & 1324.00 & 89.30 \\
4000 & 0.0709 & none & 1.80 & 1782.00 & 89.20 \\
5000 & 0.0719 & none & 1.80 & 2259.00 & 89.20 \\
6000 & 0.0732 & none & 1.80 & 2758.00 & 89.10 \\
7000 & 0.0747 & none & 1.80 & 3286.00 & 89.00 \\
8000 & 0.0766 & none & 1.80 & 3852.00 & 89.00 \\
9000 & 0.0789 & none & 1.80 & 4463.00 & 88.90 \\
10000 & 0.0817 & none & 1.80 & 5131.00 & 88.70
\end{tabular}

\section{Table 4 - Small antenna characterization data}

From this data, more was learned about the antennas and what could be expected from them. This data could have also been used to perform impedance matching. Impedance matching was not used as it was believed that the antenna would work well enough to be able to show the performance of the amplifier. The impedance was plotted against the frequency to see approximately where the antenna resonated and which frequency yielded the best results. Below is the graph of the large antenna. 


\section{Impedance vs. Frequency}

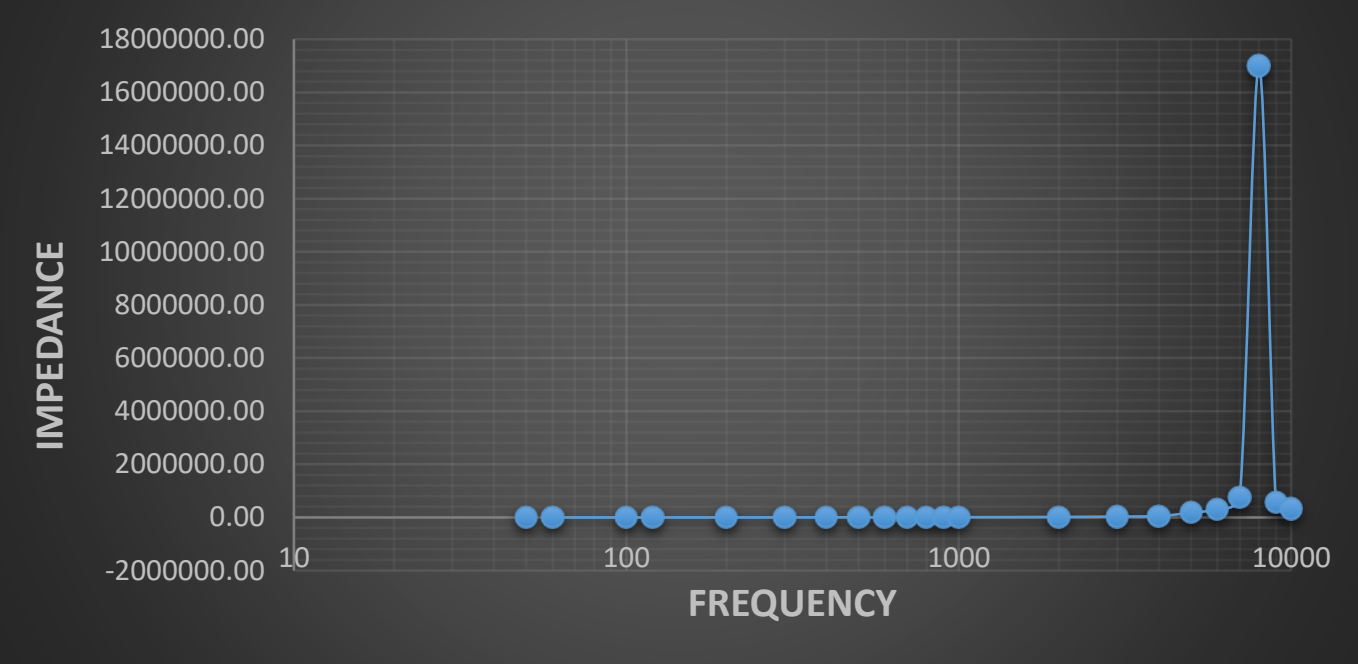

Figure 24 - Impedance vs frequency for large antenna $0 \mathrm{~Hz}-10 \mathrm{kHz}$

A second graph was generated for this data to better look at the frequencies below $1 \mathrm{kHz}$. That graph is shown below.

\section{Impedance vs. Frequency}

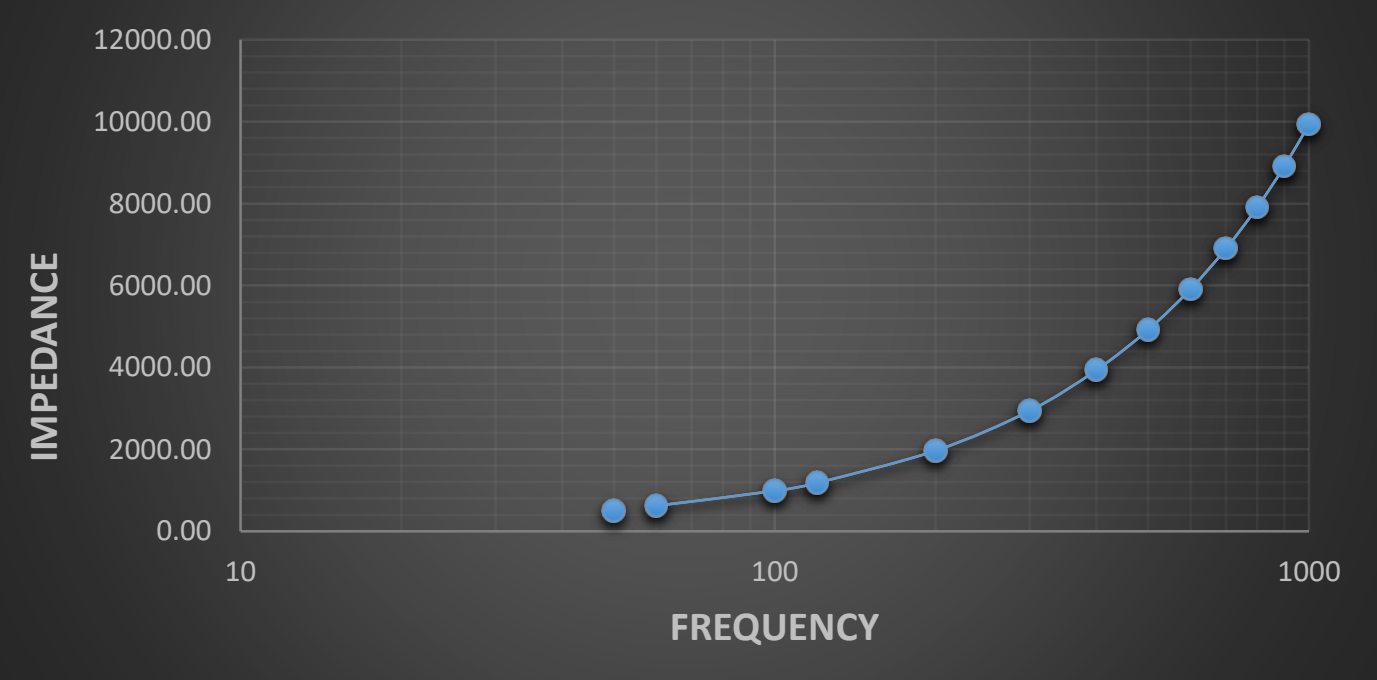

Figure 25 - Impedance vs frequency for large antenna $0 \mathrm{~Hz}-1 \mathrm{kHz}$

It can be seen from the graph that the antenna resonated at about $8 \mathrm{kHz}$. This was not in the range of frequencies that was targeted and was not the focus of this experiment but was believed to be 
low enough that it could have been useful for testing purposes. This next graph shows what the plot looked like for the small antenna.

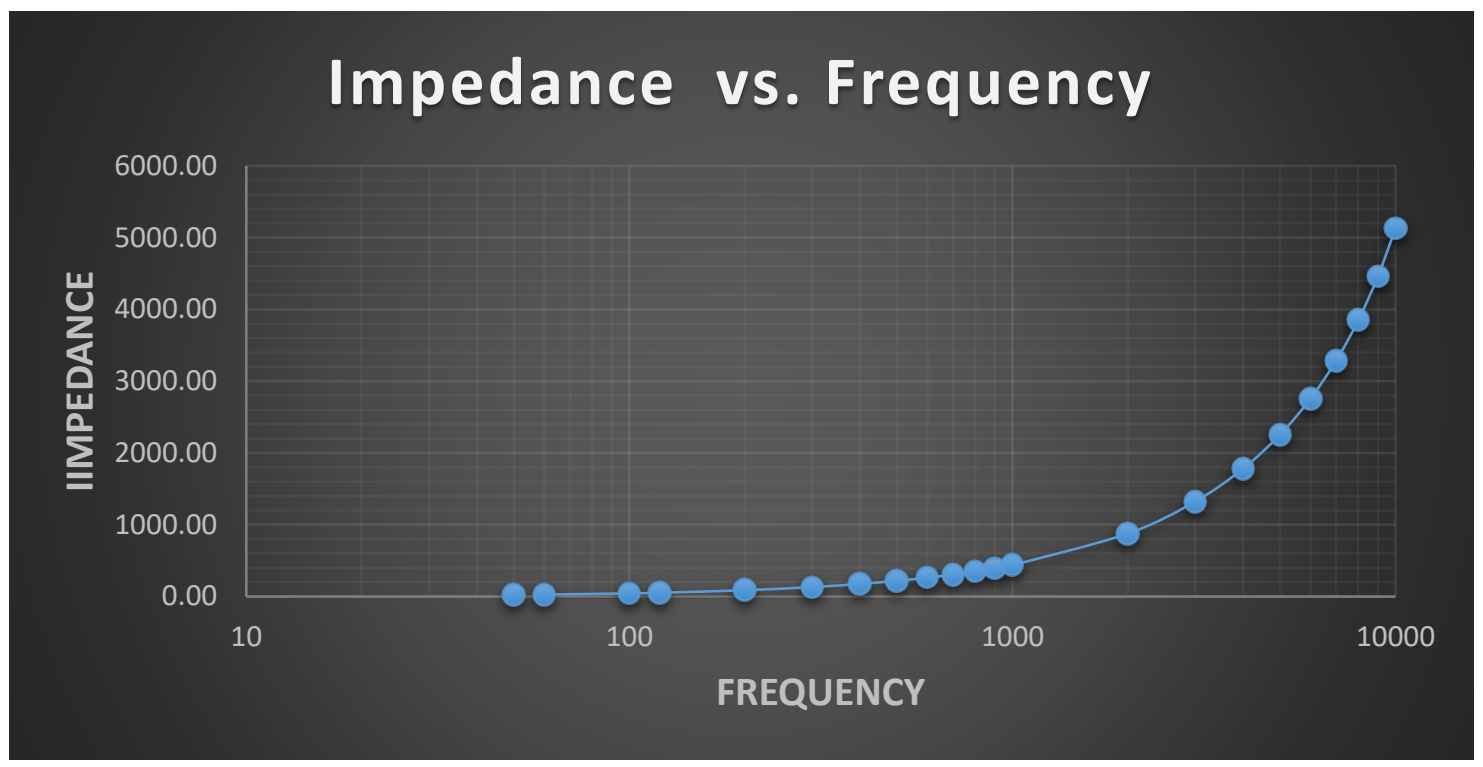

Figure 26 - Impedance vs frequency for small antenna $0 \mathrm{~Hz}-10 \mathrm{kHz}$

A second graph was generated for this data to better look at the frequencies below $1 \mathrm{kHz}$. That graph is shown below.

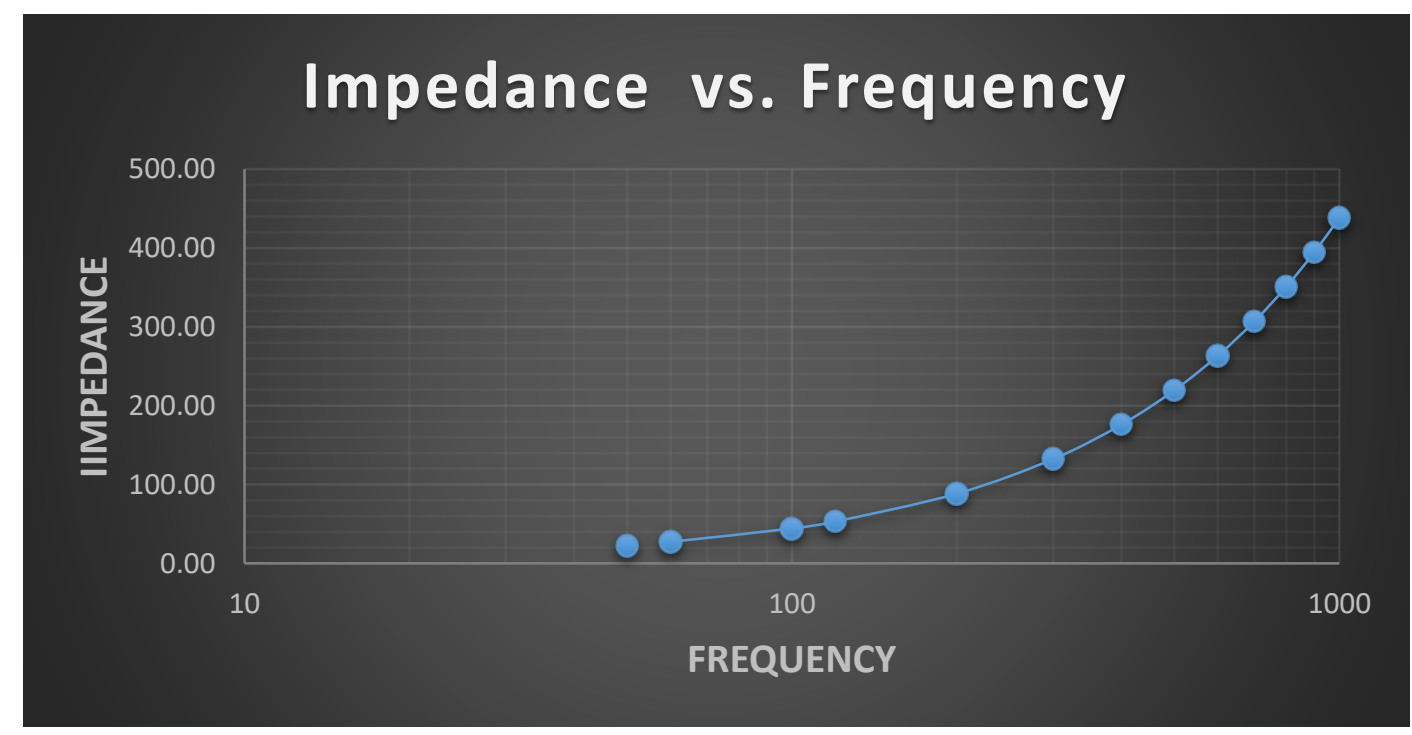

Figure 27 - Impedance vs frequency for small antenna $0 \mathrm{~Hz}-1 \mathrm{kHz}$

It can be seen from the graph of the small antenna that the antenna never appeared to have reached the resonant frequency, but it was greater than $12 \mathrm{kHz}$. Since the system needed a way to transmit test signals, this antenna was used for that. 


\section{CHAPTER 4: BENCH TESTING AND ANALYSIS}

\subsection{Bench Test Results}

To validate the system a set of tests had to be devised to ensure that the system performed in the manner that was expected. To achieve the best test possible, the system was broken down into three parts or tests. The first test was the amplifier bench test. In this test, a low voltage signal was input that was like the expected signal that was received by the antenna. The second test tested the antenna's signal reception capabilities. To do this was challenging, but a signal was transmitted through the antenna of similar size and capabilities and then the signal that was received by the antenna was measured. Lastly, all parts were combined, the antenna and amplifier, into one cohesive unit. The entire system was tested using the same antenna from the second test to transmit a signal and then the output was viewed after amplification. Three methods were used to view and measure the output. The first method was the traditional oscilloscope. Another method was using a digital oscilloscope which was built into the same box that the amplifier resided in. The digital oscilloscope was called the PoScope which was sold by PoLabs. The last method was using the sound card in a Dell Latitude E6320 and a program called Spectrum Lab was used help visualize the data.

\subsubsection{Amplifier Bench Test}

For this test, the goal was to determine if the amplifier performed the way it was designed and if the design was able to amplify a small signal without too much distortion. The test setup consisted of a function generator, dual DC power supply, oscilloscope, the circuit, a prototyping board and a PC running Microsoft Excel that was used to record all measurements. The image below shows the entire test setup. 


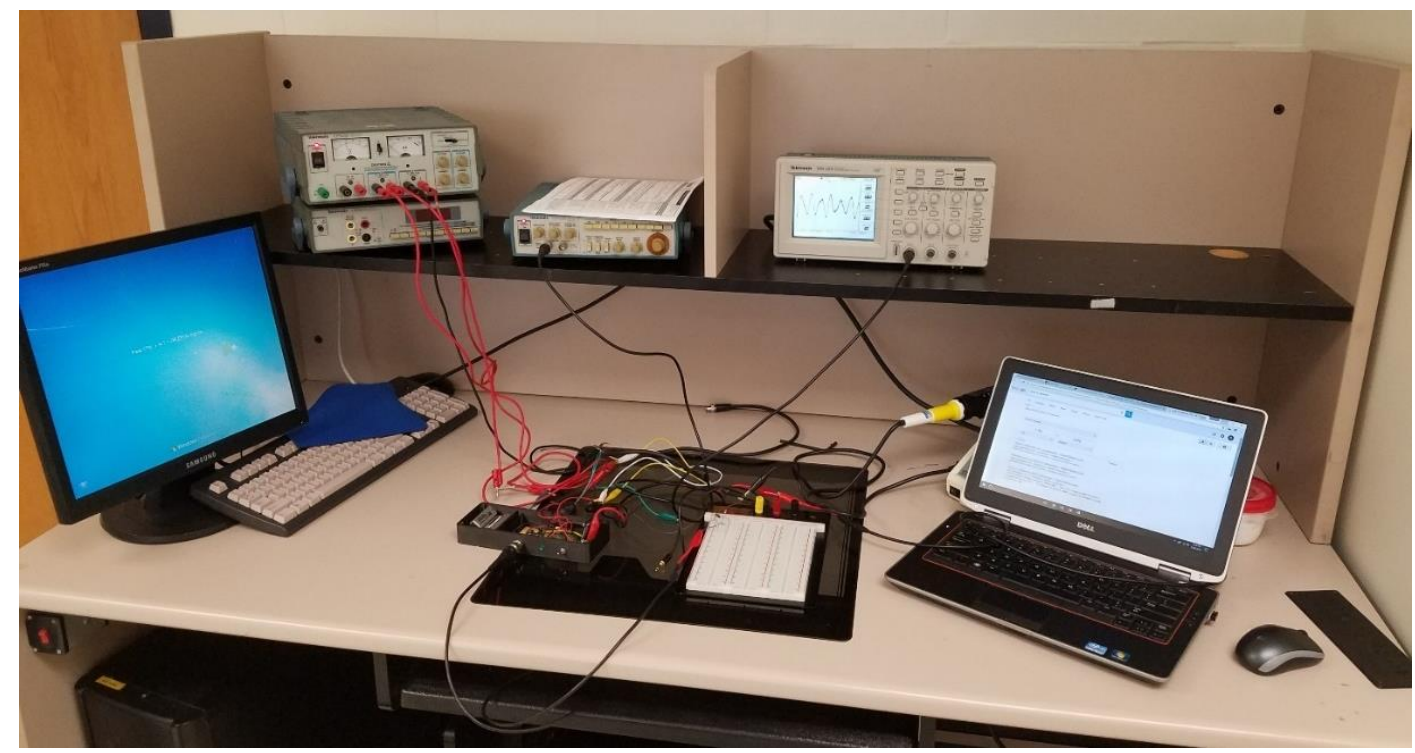

Figure 28 - Amplifier test setup

For the test, positive and negative $20 \mathrm{~V}$ was supplied at the corresponding terminals on the amplifier configuration for the voltage supply. The positive input of the amplifier received the signal from the function generator and negative input of the amplifier was connected to ground. The output was then connected to the oscilloscope. For the initial try of the test, a sinusoidal input was given with a voltage of $79.2 \mathrm{mV}$ at $7.349 \mathrm{~Hz}$. Below is a capture from the oscilloscope.

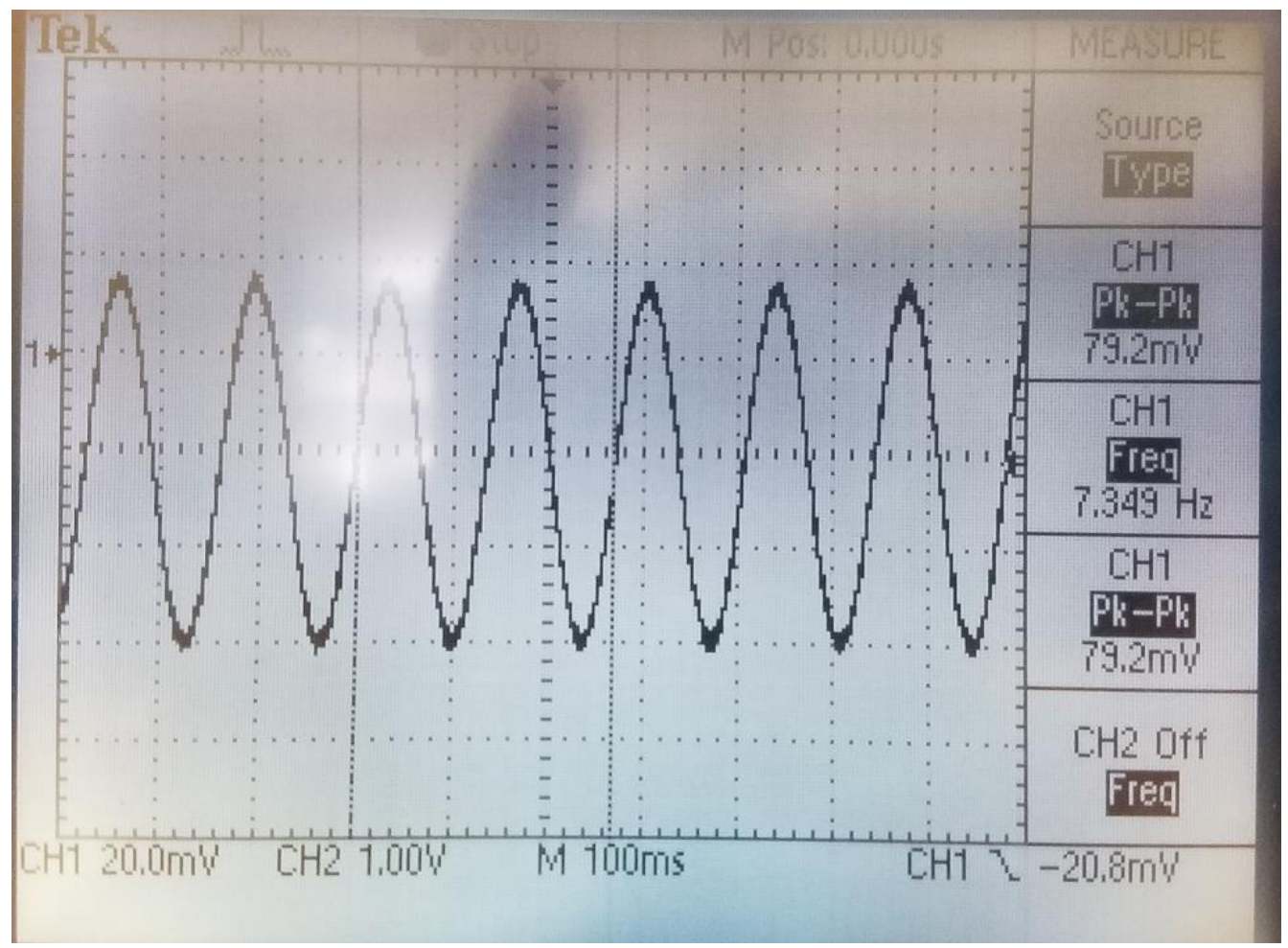

Figure 29 - Input signal to amplifier 
The input signal was passed through the circuit and an output signal was received, below is a capture of that signal.

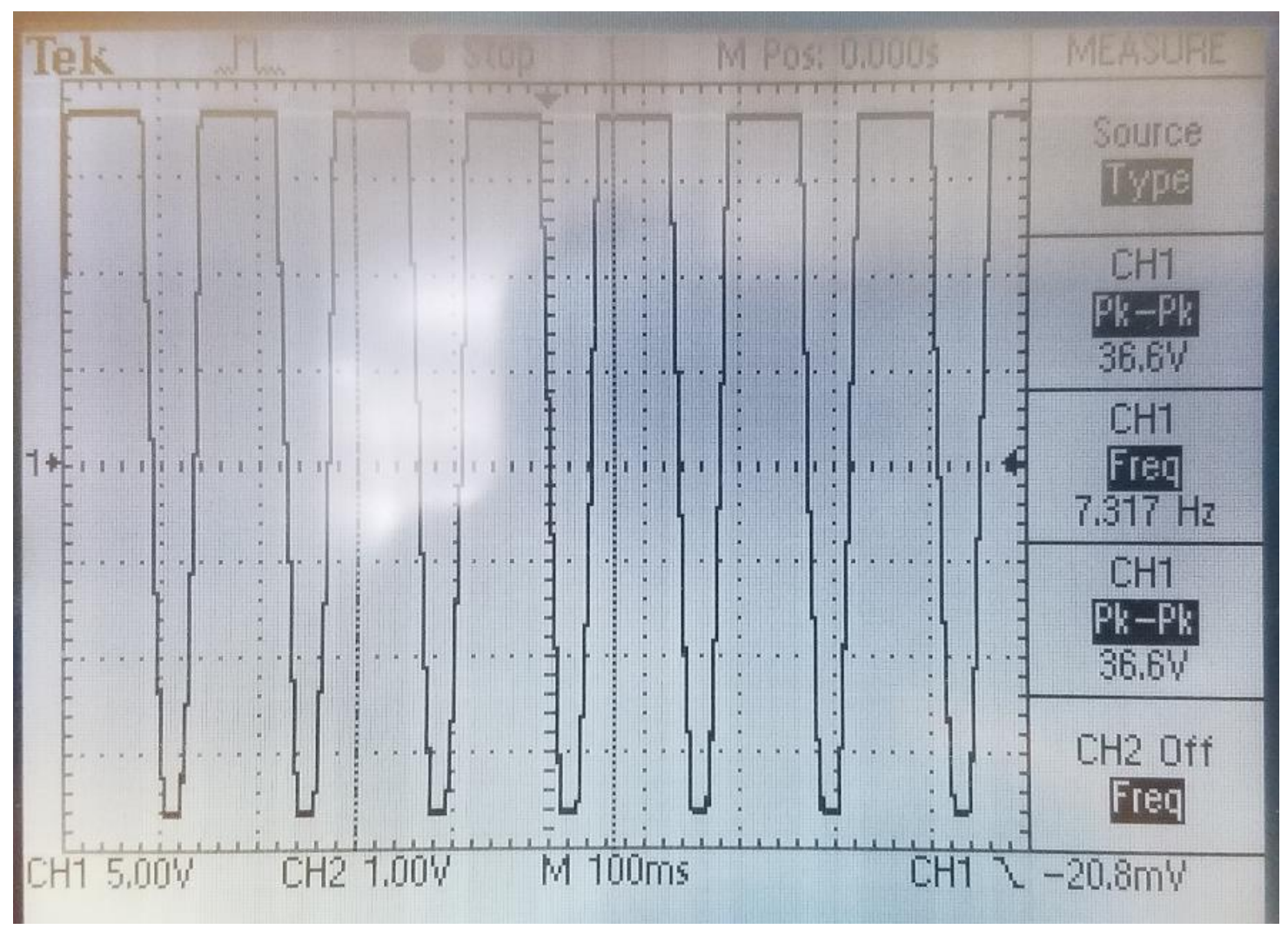

Figure 30 - Saturated output signal from amplifier

From the capture, it can be seen that the output voltage is $36.6 \mathrm{~V}$ at $7.317 \mathrm{~Hz}$. There were two things noticed from the capture. The first was the frequency difference. This was caused by noise present on the amplified signal that was generated by the circuit and due to the way this particular oscilloscope captured the frequency (weighted average of all frequencies and signal levels) this was confirmed by seeing that the frequency of the input signal was output but noise was definitely added. The second thing that was noticed was that the output was not a true sinusoid wave. This was due to the amplfier saturating and it caused the output to be clipped. The amplifier can only handle so much output which was dictated by the voltage given to the power terminals and, in this case, it was corrected by lowering the input signal level to avoid this clipping issue.

To get the signal level to a voltage that would not cause clipping and was closer to what was expected to be seen by the antenna and due to the limited low power capabilities of the function generator, a voltage divider was used to get that signal down to a reasonable level. Below is the schematic for the voltage divider. 


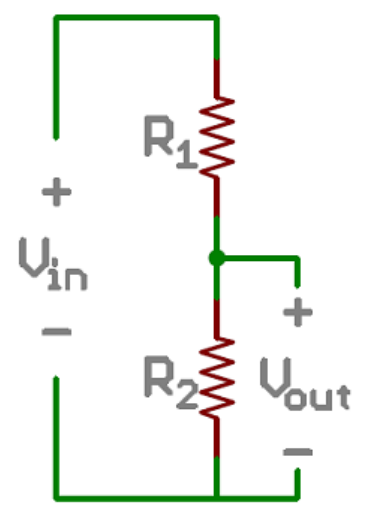

Figure 31 - Voltage divider circuit

For this case, the Vin voltage was $1.06 \mathrm{~V}$ and the desired input signal was to be around $10 \mathrm{mV}$. The signal level was around $10 \mathrm{mV}$ because it was expected to be low enough to not allow clipping via the amplifier. From the voltage divider circuit, the values that were needed were R1 and R2. The output that was desired was determined using the formula below.

$$
V_{\text {out }}=V_{\text {in }} * \frac{R_{2}}{R_{1}+R_{2}}
$$

From this formula, it was solved for $\mathrm{R}_{1}$ and $\mathrm{R}_{2}$. Since the $\mathrm{V}_{\text {out }}$ did not need to be exactly $1 \mathrm{mV}$ several values were plugged into the equation based on resistors values the are more common and readily available. For the test, the resistors that were available were $1 \mathrm{Mohm}$ and $1 \mathrm{kohm}$ which were used as $R_{1}$ and $R_{2}$ respectively. With these values, an expected $V_{\text {out }}$ of approximately 1.06 $\mathrm{mV}$ was obtained. The equipment was not sensitive enough to accurately measure this small of a signal so the amplified signal was expected to be around $1.3 \mathrm{~V}$. If the signal was not present or would have been weaker than expected, it would have been assumed that there was an issue with the amplifier since it was known that it works for higher signals and that the voltage divider worked for all signals regardless of frequency and magnitude.

With a signal that was now low enough, the full tests could be performed so that the performance of the amplifier could be seen. Below is a block diagram showing the full test setup. 


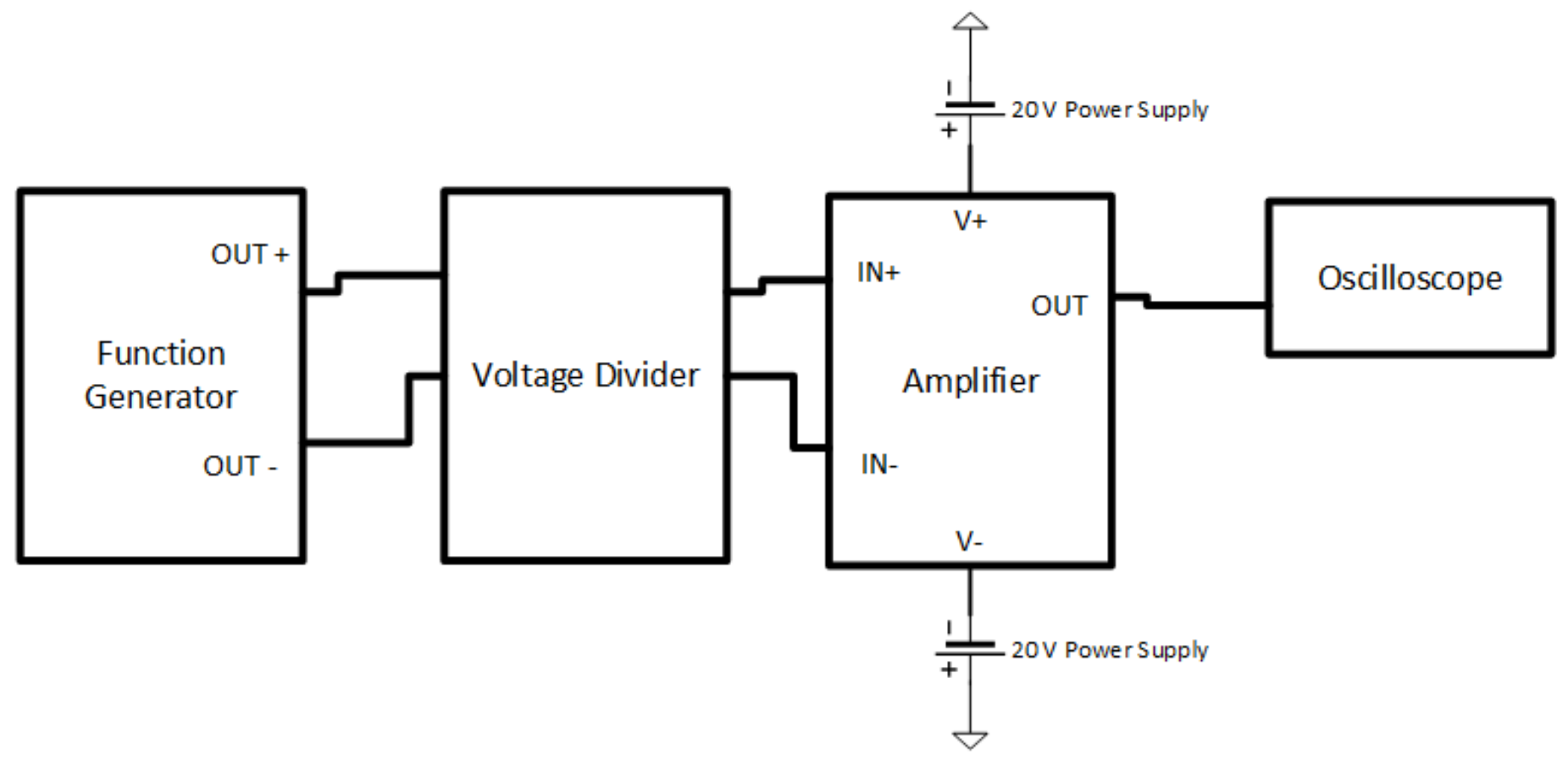

Figure 32 - Revised amplifier test setup as block diagram

The full test did a logarithmic sweep of all frequencies from $10 \mathrm{~Hz}$ up to $3 \mathrm{kHZ}$. The test measured the original signal generated by the function generator and the output signal created by the amplifier. To determine the gain of the system, the following formula was used.

$$
G=\frac{V_{o u t} *\left(R_{1}+R_{2}\right)}{V_{\text {in }} * R_{2}}
$$

Since the values for $R_{1}$ and $R_{2}$ were known, the values substituted into the equation to make the formula simpler.

$$
G=1001 * \frac{V_{\text {out }}}{V_{\text {in }}}
$$

With this, the tests were performed in full. Sample outputs from the oscilloscope are shown below. 


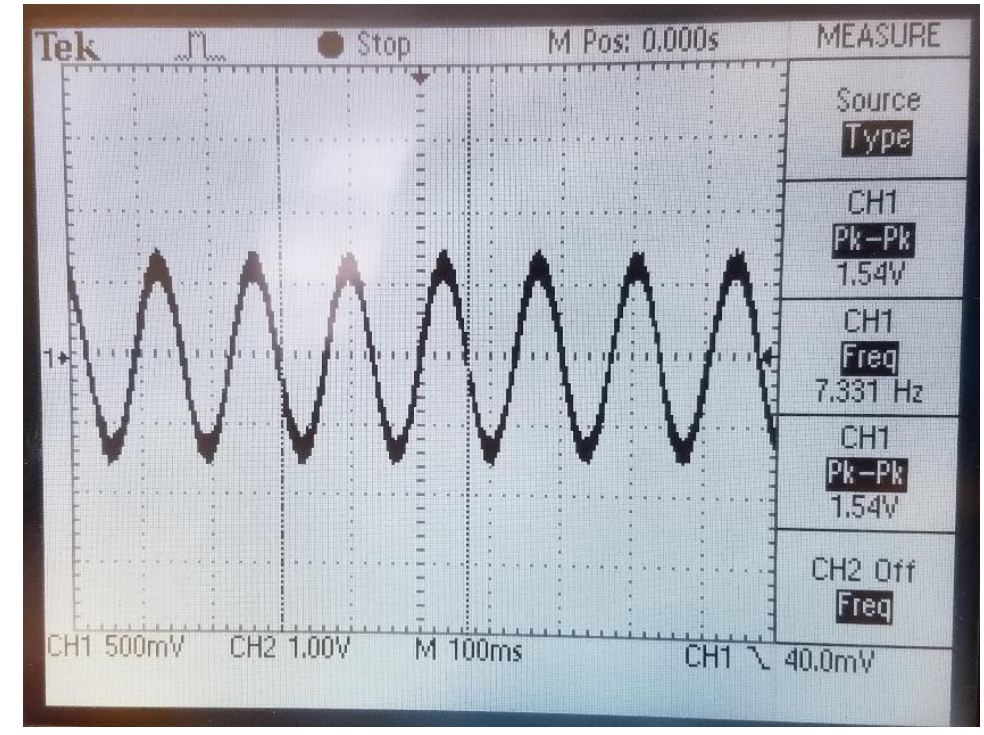

Figure 33 - Output signal from amplifier with new test setup

The image above shows the output of the amplifier at $7.331 \mathrm{~Hz}$ which was roughly equivalent to the desired frequency. It can be seen that the output voltage is $1.54 \mathrm{~V}$.

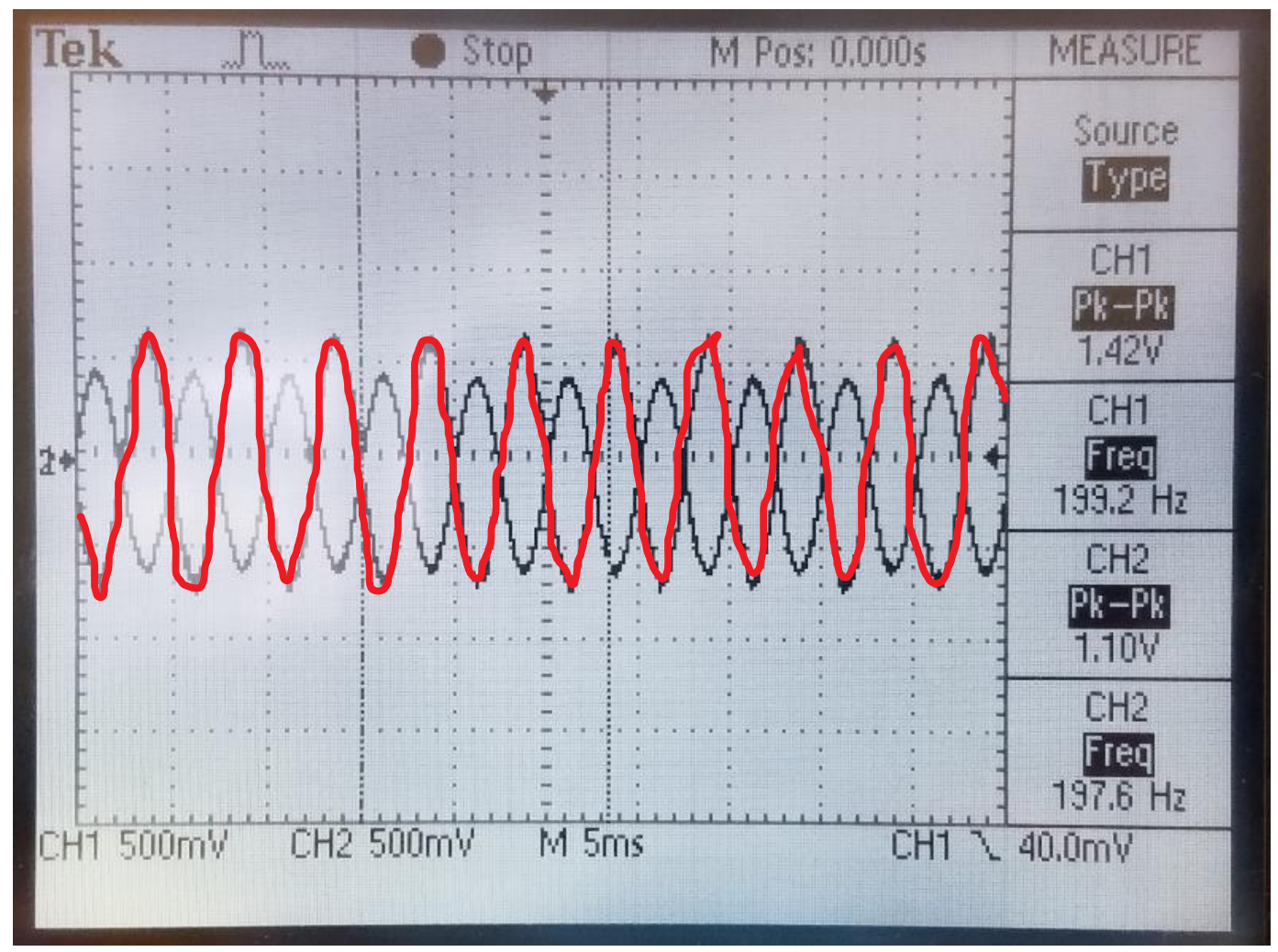

Figure 34 - Input signal before voltage division (black) and output from amplifier (red) 
In this image, the input voltage and the output voltage can be seen in the same view $\mathrm{CH} 1$ or the red line corresponds to the output and $\mathrm{CH} 2$ or the black line corresponds to the input. The input voltage is not the signal that was input into the amplifier but is the signal that was input into the voltage divider before being sent to the amplifier. The output signal is the output directly from the amplifier.

The following table shows the full results of the tests. The analysis of these results and all other tests follows this section in section 4.2.

\begin{tabular}{|c|c|c|c|c|c|}
\hline Frequency & $\begin{array}{l}\text { Vin before voltage } \\
\text { divider (mv) }\end{array}$ & $\begin{array}{l}\text { Vin after voltage } \\
\text { divider (mv) }\end{array}$ & Vout (mV) & Gain & Gain (dB) \\
\hline 10 & 1060 & 1.058941 & 1560 & 1473.1698 & 63.36505621 \\
\hline 20 & 1060 & 1.058941 & 1460 & 1378.7358 & 62.78962136 \\
\hline 30 & 1080 & 1.078921 & 1440 & 1334.6667 & 62.50745628 \\
\hline 40 & 1080 & 1.078921 & 1420 & 1316.1296 & 62.38597333 \\
\hline 50 & 1080 & 1.078921 & 1400 & 1297.5926 & 62.26276715 \\
\hline 60 & 1080 & 1.078921 & 1440 & 1334.6667 & 62.50745628 \\
\hline 70 & 1080 & 1.078921 & 1440 & 1334.6667 & 62.50745628 \\
\hline 80 & 1080 & 1.078921 & 1420 & 1316.1296 & 62.38597333 \\
\hline 90 & 1080 & 1.078921 & 1420 & 1316.1296 & 62.38597333 \\
\hline 100 & 1100 & 1.098901 & 1420 & 1292.2 & 62.22659473 \\
\hline 200 & 1100 & 1.098901 & 1420 & 1292.2 & 62.22659473 \\
\hline 300 & 1100 & 1.098901 & 1420 & 1292.2 & 62.22659473 \\
\hline 400 & 1080 & 1.078921 & 1400 & 1297.5926 & 62.26276715 \\
\hline 500 & 1080 & 1.078921 & 1400 & 1297.5926 & 62.26276715 \\
\hline 600 & 1080 & 1.078921 & 1400 & 1297.5926 & 62.26276715 \\
\hline 700 & 1080 & 1.078921 & 1380 & 1279.0556 & 62.13778817 \\
\hline 800 & 1080 & 1.078921 & 1400 & 1297.5926 & 62.26276715 \\
\hline 900 & 1080 & 1.078921 & 1340 & 1241.9815 & 61.88230241 \\
\hline 1000 & 1080 & 1.078921 & 1320 & 1223.4444 & 61.75168506 \\
\hline 2000 & 1080 & 1.078921 & 1340 & 1241.9815 & 61.88230241 \\
\hline 3000 & 1120 & 1.118881 & 1380 & 1233.375 & 61.82190282 \\
\hline 4000 & 1120 & 1.118881 & 1340 & 1197.625 & 61.56641706 \\
\hline 5000 & 1120 & 1.118881 & 1200 & 1072.5 & 60.60794602 \\
\hline 6000 & 1120 & 1.118881 & 1180 & 1054.625 & 60.46196124 \\
\hline 7000 & 1120 & 1.118881 & 1080 & 965.25 & 59.69279621 \\
\hline 8000 & 1120 & 1.118881 & 1080 & 965.25 & 59.69279621 \\
\hline 9000 & 1100 & 1.098901 & 1000 & 910 & 59.18082785 \\
\hline 10000 & 1100 & 1.098901 & 940 & 855.4 & 58.64338492 \\
\hline 20000 & 1140 & 1.138861 & 640 & 561.96491 & 54.994184 \\
\hline 30000 & 1140 & 1.138861 & 480 & 421.47368 & 52.49540927 \\
\hline
\end{tabular}

Table 5 - Amplifier bench test results 


\subsubsection{Antenna Bench Test}

The test for the antenna was very simple since it was not the focus of this experiment. The test setup as a block diagram is shown below.

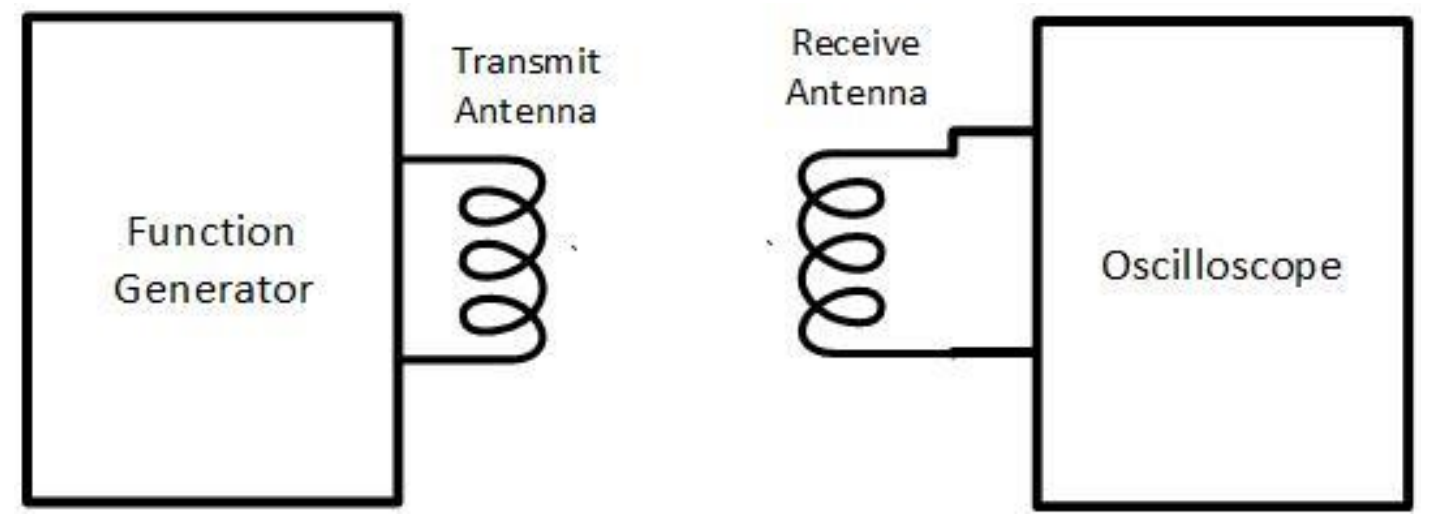

Figure 35 - Antenna bench test setup as block diagram

To examine the performance of the antennas without the amplifier, the antennas were placed approximately 1 foot from each other and the frequency range of $10 \mathrm{~Hz}-10 \mathrm{kHz}$ was input into the transmit antenna. The signal was then recorded after being received by the receive antenna. Below is the capture of the signal on the large loop, on the oscilloscope. 


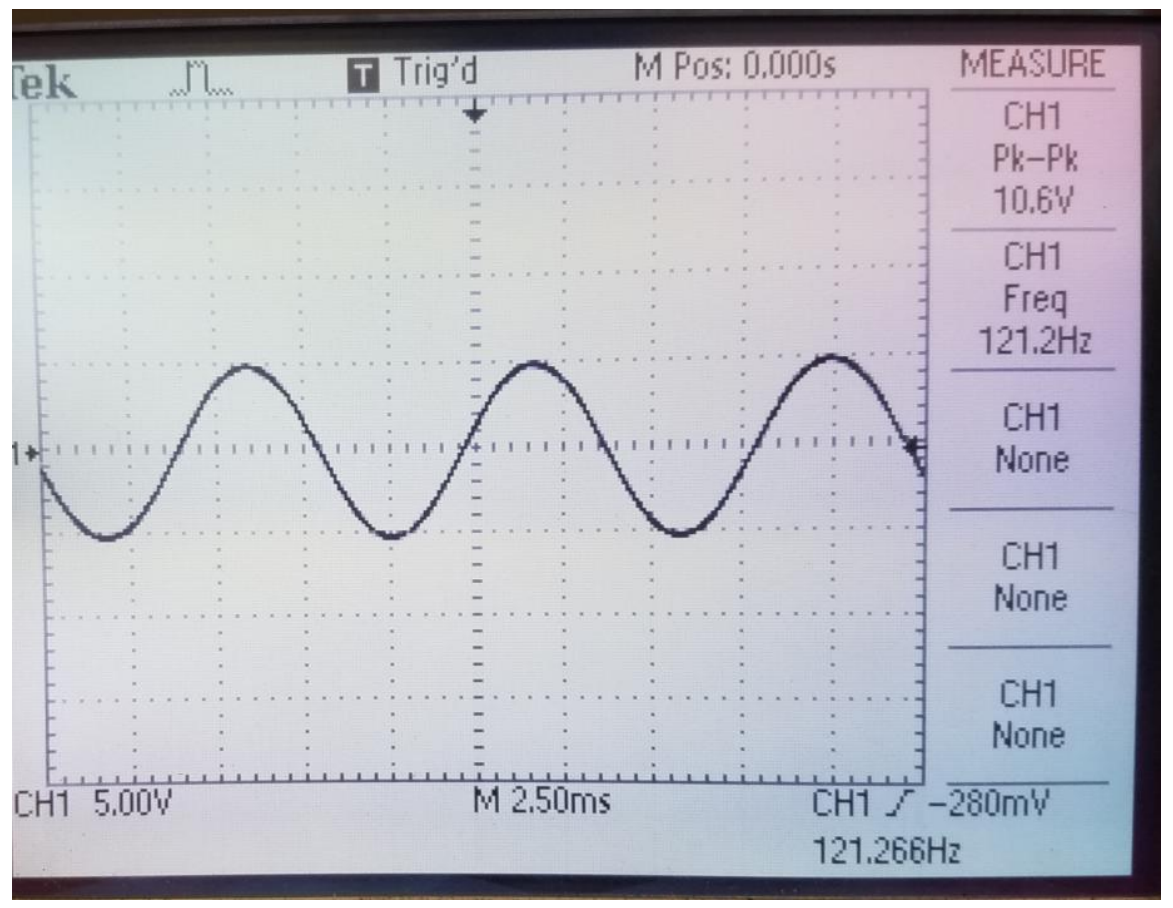

Figure 36 - Sample input signal for transmit antenna

For the reception, the antenna was hooked directly up to oscilloscope so the signal could be viewed. Below is a screen capture of what the antenna received as was seen by the oscilloscope.

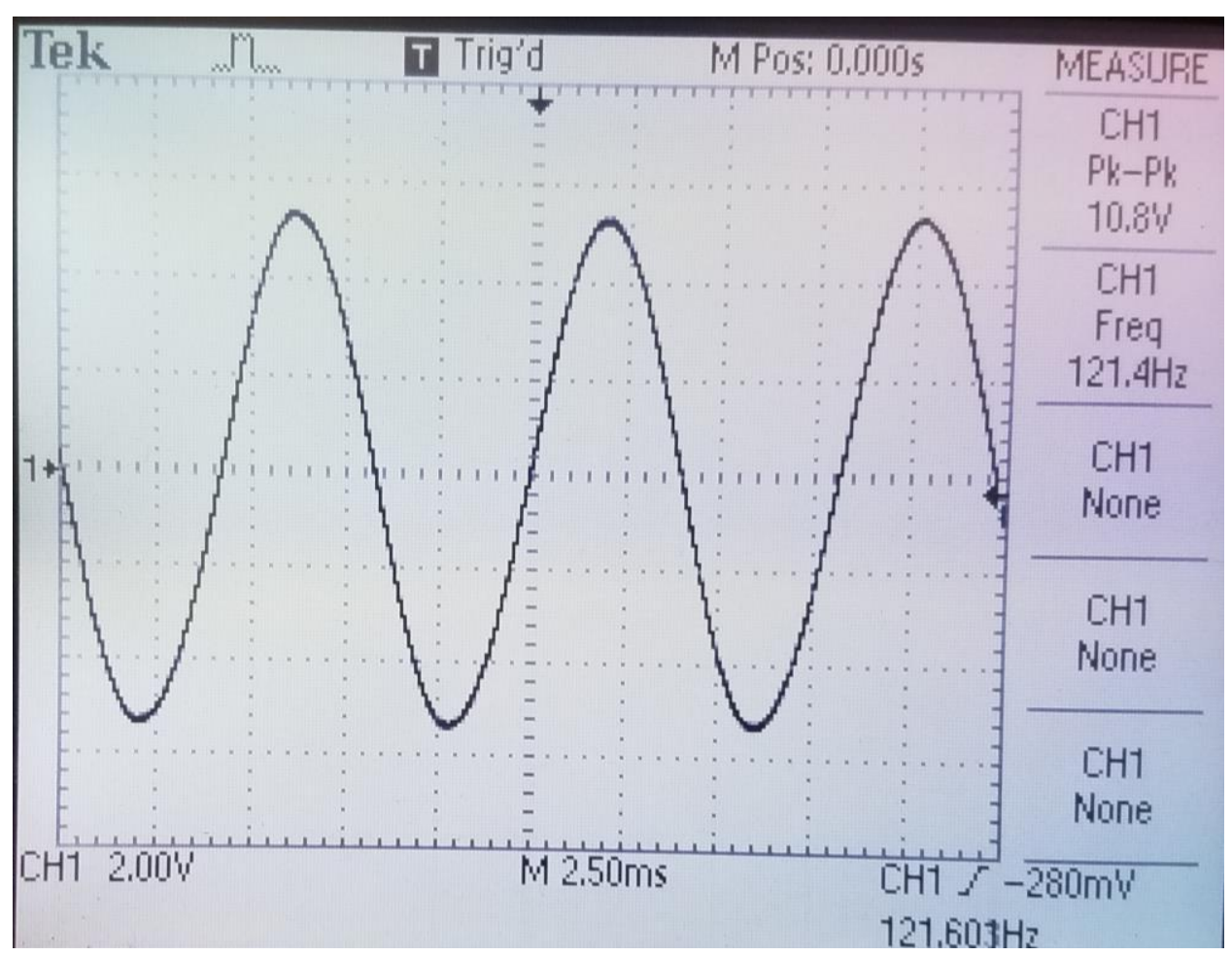

Figure 37 - Received signal by receive antenna 
The data from the entire test is shown below.

\begin{tabular}{|c|c|c|c|c|}
\hline Frequency $(\mathrm{Hz})$ & Transmit Input Voltage (V) & Received Output Voltage (V) & Gain & Gain $(\mathrm{dB})$ \\
\hline 10 & 1.8 & 1.5 & 0.8 & -1.6 \\
\hline 20 & 3.4 & 2.2 & 0.6 & -3.8 \\
\hline 30 & 6.0 & 3.6 & 0.6 & -4.4 \\
\hline 40 & 7.8 & 4.4 & 0.6 & -5.0 \\
\hline 50 & 9.0 & 5.0 & 0.6 & -5.1 \\
\hline 60 & 10.6 & 5.6 & 0.5 & -5.5 \\
\hline 70 & 11.8 & 6.4 & 0.5 & -5.3 \\
\hline 80 & 13.0 & 7.2 & 0.6 & -5.1 \\
\hline 90 & 14.0 & 7.6 & 0.5 & -5.3 \\
\hline 100 & 14.8 & 8.0 & 0.5 & -5.3 \\
\hline 200 & 19.6 & 10.4 & 0.5 & -5.5 \\
\hline 300 & 21.0 & 11.2 & 0.5 & -5.5 \\
\hline 400 & 21.8 & 11.6 & 0.5 & -5.5 \\
\hline 500 & 22.0 & 11.8 & 0.5 & -5.4 \\
\hline 600 & 22.2 & 12.0 & 0.5 & -5.3 \\
\hline 700 & 22.2 & 12.0 & 0.5 & -5.3 \\
\hline 800 & 22.2 & 12.2 & 0.5 & -5.2 \\
\hline 900 & 22.4 & 12.2 & 0.5 & -5.3 \\
\hline 1000 & 22.6 & 12.2 & 0.5 & -5.4 \\
\hline 2000 & 22.6 & 13.0 & 0.6 & -4.8 \\
\hline 3000 & 23.0 & 15.0 & 0.7 & -3.7 \\
\hline 4000 & 23.0 & 19.0 & 0.8 & -1.7 \\
\hline 5000 & 22.8 & 26.0 & 1.1 & 1.1 \\
\hline 6000 & 22.8 & 44.0 & 1.9 & 5.7 \\
\hline 7000 & 23.0 & 126.0 & 5.5 & 14.8 \\
\hline 8000 & 23.0 & 30.0 & 1.3 & 2.3 \\
\hline 9000 & 23.0 & 16.0 & 0.7 & -3.2 \\
\hline 10000 & 23.0 & 10.0 & 0.4 & -7.2 \\
\hline
\end{tabular}

\section{Table 6 - Antenna test data}

The reception of the signals confirmed that the signals were received with the antenna and that the antenna would work for the system to assist in testing the amplifier with a non-perfect source that was close to what would occur in practice. The antennas were also moved closer together and further apart to ensure that the signal was still received and this was confirmed to work to a point. When the antenna was more than about 4.5 feet apart the signal became too faint to detect via the oscilloscope, this is where the amplifier would become useful. 


\subsubsection{System Bench Test}

The primary focus of this test was to determine if the amplifier could amplify a small/weak signal to be strong enough to be picked up by a PC sound card so that further processing might be done on the signal. To test these several things had to be in place. The first thing needed was a signal to detect. This signal came from one of the antennas that were built (the one with fewer windings). The receive antenna was placed about 5 feet from the reception antenna. This distance is short due to impedance mismatches and not being able to transmit a strong enough signal with just a basic function generator. This distance is far enough apart so that the antenna appears to only hear the noise when not connected to the amplifier. The figure below shows the test setup as a block diagram.
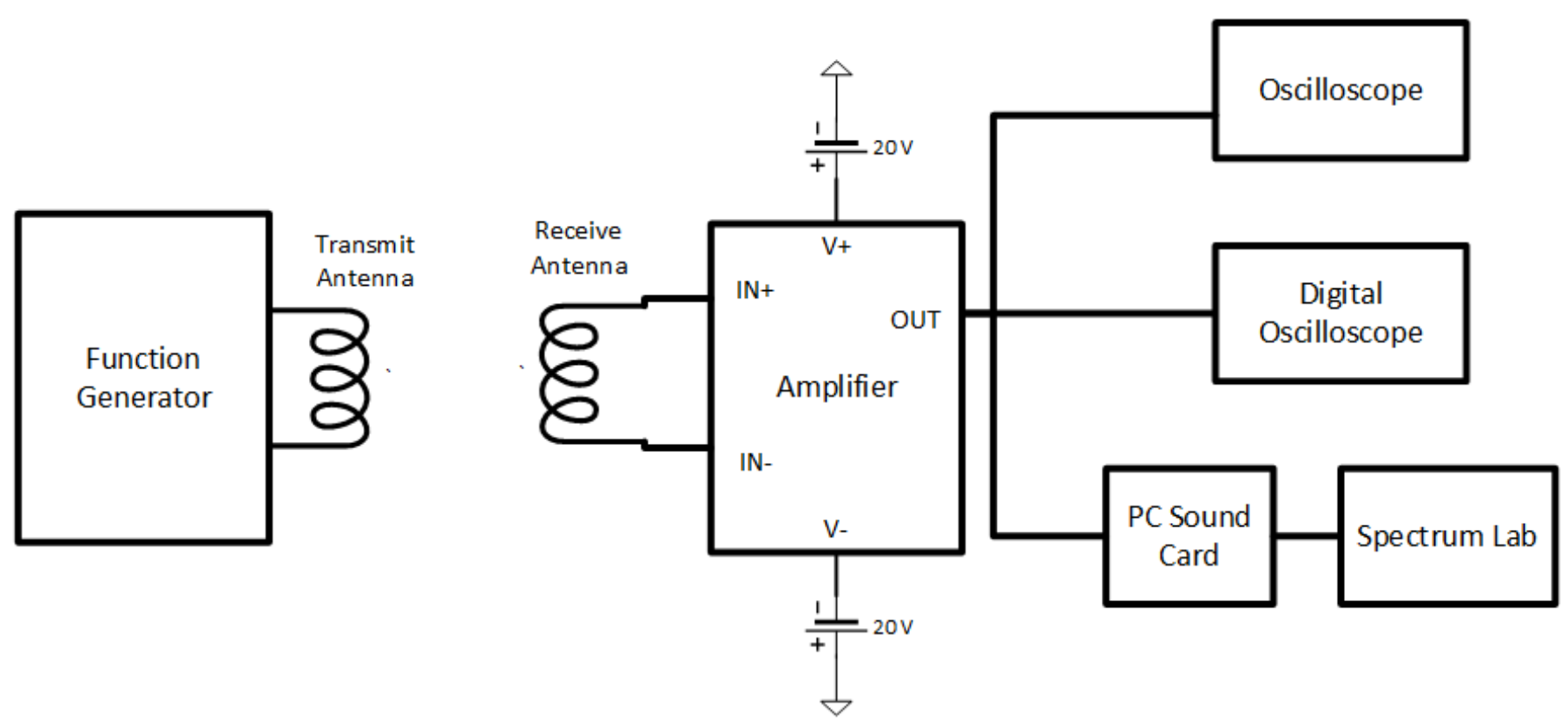

Figure 38 - System bench test setup as block diagram

For the test, several frequencies were transmitted and were received by the system. The figure below shows a screen shot of SpectrumLab and what was the test was performed. At the top of the figure, there is a real-time view of the signal and below is a waterfall display, which essentially records the signal through time and displays it. The top of the waterfall would be the most recent time and the bottom of the waterfall would be the oldest time visible (typically set in an options menu). 


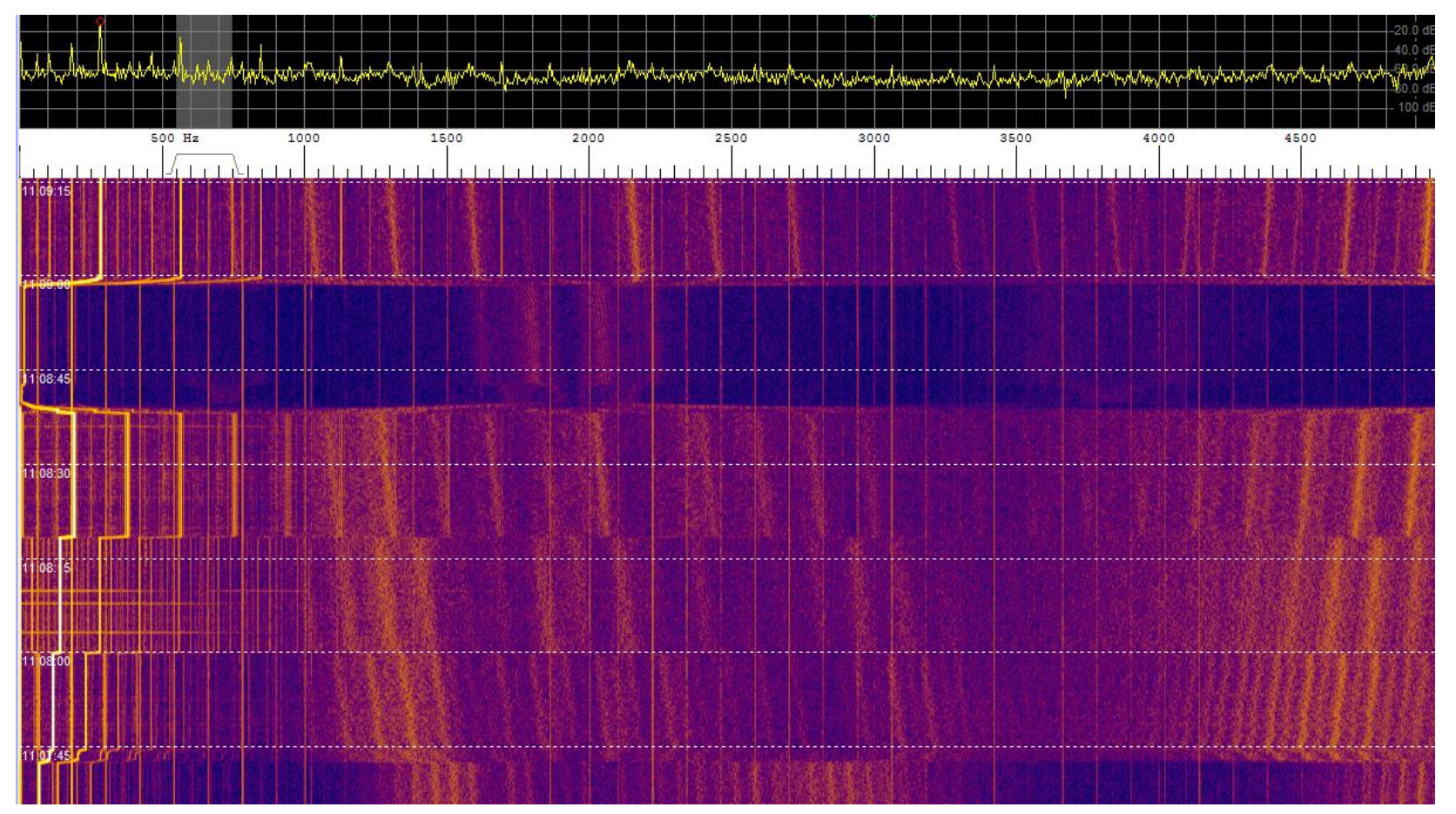

Figure 39 - Spectrum lab output, showing waterfall display and oscilloscope functionality

From the figure, it can be seen how the signals moved up in frequency and through time. Several of the harmonics of the signal can also be seen in the figure. Using this display, several different data points were recorded to assist in determining how the system performed. For the test, the transmit voltage was recorded and then on the receive end the signal level after amplification was recorded in decibels. Below are two tables of data one for transmit and one for receive.

\begin{tabular}{|r|r|r|r|}
\hline Frequency & Transmit Voltage $(\mathrm{V})$ & Signal Level After Amplifier (dB) & \multicolumn{1}{c|}{ Signal Level (V) } \\
\hline 12 & 20 & -24.0 & 0.06 \\
\hline 24 & 20 & -20.0 & 0.10 \\
\hline 78 & 20 & -13.0 & 0.22 \\
\hline 122 & 20 & -9.6 & 0.33 \\
\hline 139 & 20 & -8.4 & 0.38 \\
\hline 185 & 20 & -9.6 & 0.33 \\
\hline 279 & 20 & -7.2 & 0.44 \\
\hline 888 & 20 & -7.2 & 0.44 \\
\hline 1500 & 20 & -6.0 & 0.50 \\
\hline 2500 & 20 & -1.2 & 0.87 \\
\hline 3250 & 20 & 0.0 & 1.00 \\
\hline 4000 & 20 & 0.0 & 1.00 \\
\hline 10000 & 20 & 0.0 & 1.00 \\
\hline
\end{tabular}

Table 7 - System test data 
From the table, a signal was received at all frequencies of interest. This confirms that the system worked and that the amplifier acted as expected. The analysis section goes more in depth breaking down these results.

\subsection{Analysis of Bench Test Results}

The following sections analyze the results from above showing how the data obtained was used to inform the decision on whether the system was successful or not.

\subsubsection{Amplifier analysis}

From the results in the previous section, the data was used to create the graph below to help illustrate how the amplifier performed.

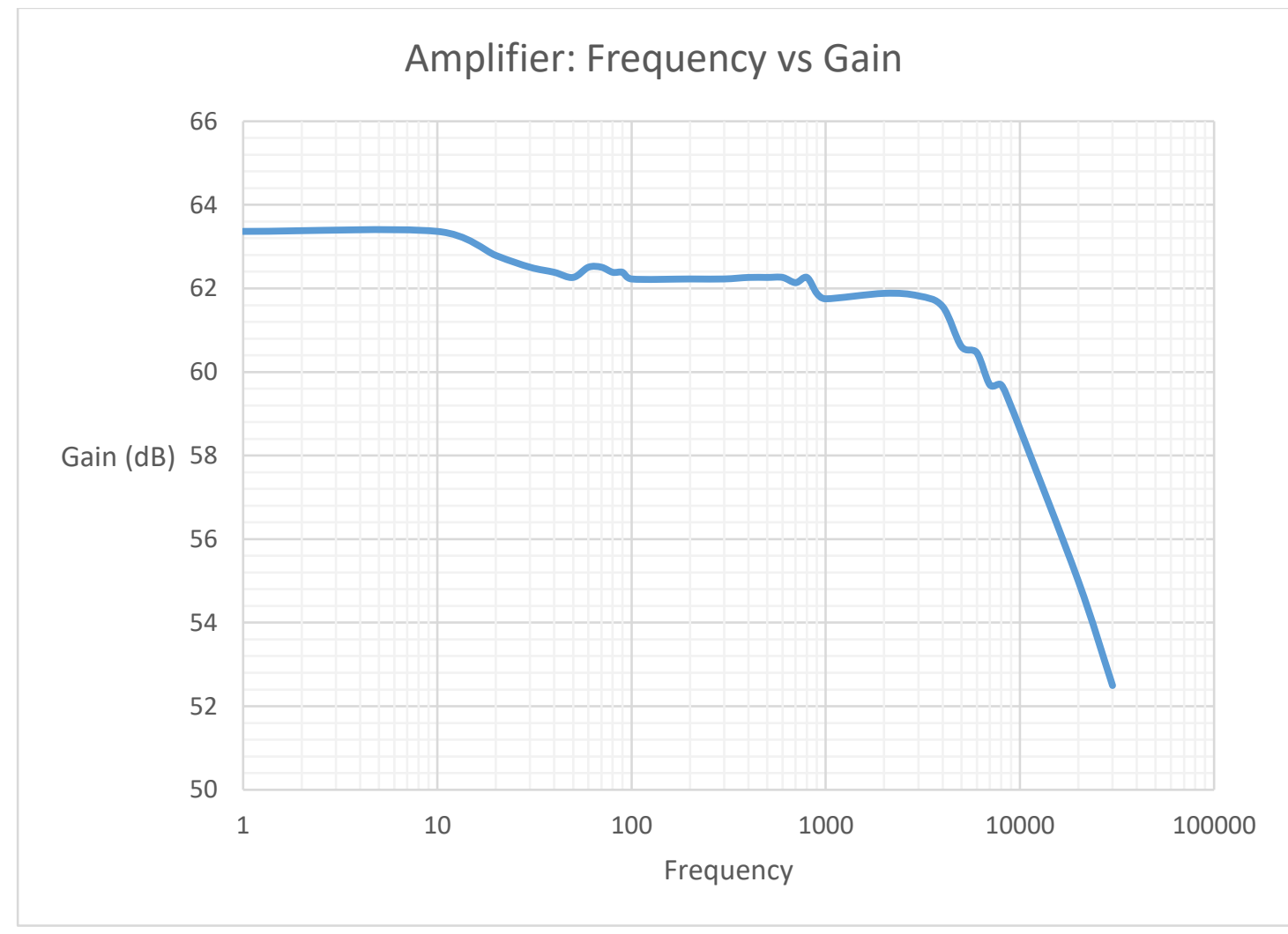

Figure 40 - Amplifier bench test frequency vs gain plot

Examining the data closer for frequencies less than $1000 \mathrm{~Hz}$ yields the graph below. 


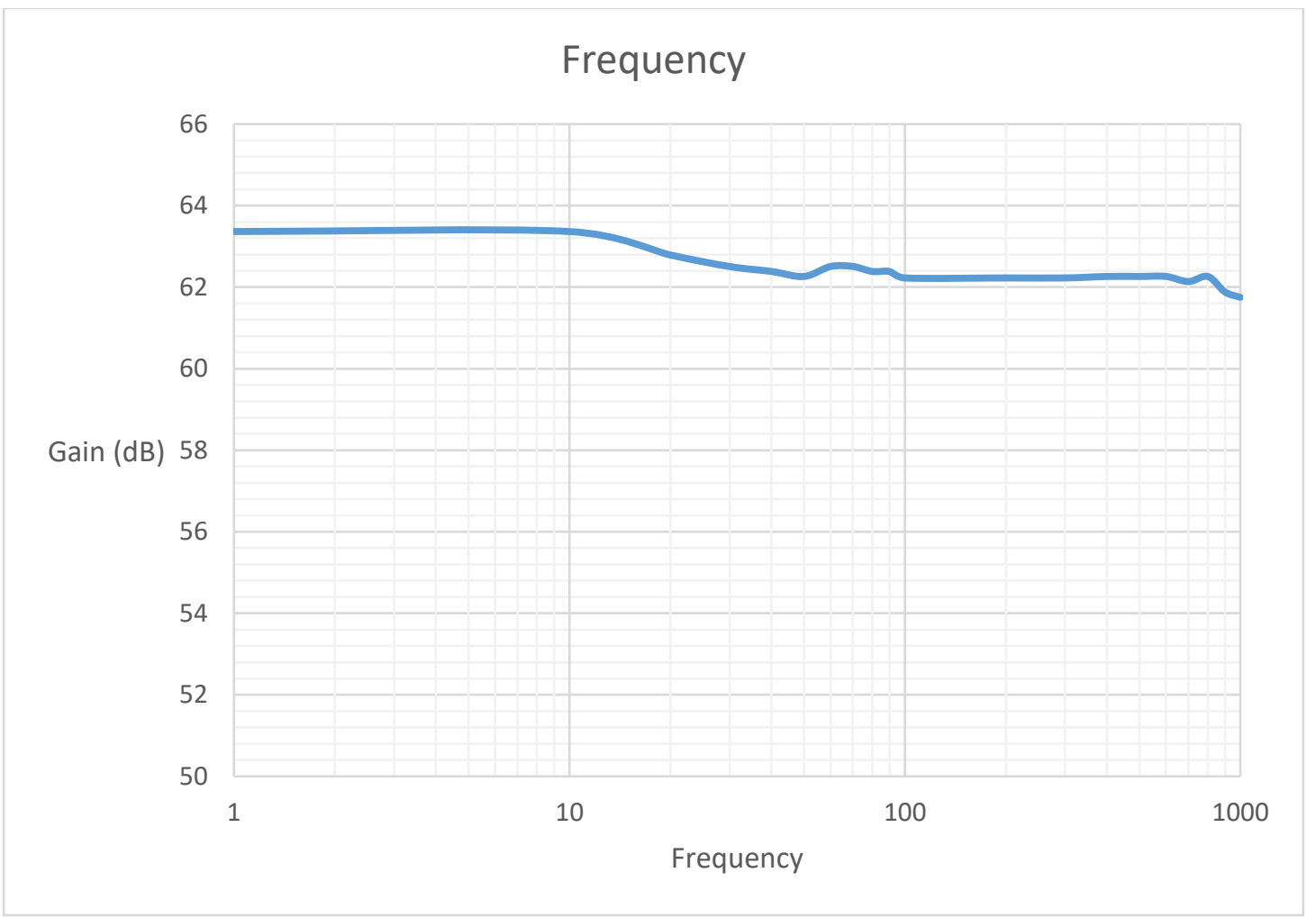

Figure 41 - Amplifier bench test frequency vs gain plot $(1 \mathrm{~Hz}-1000 \mathrm{~Hz})$

From the graph, the gain (signal generator output to amplifier output) along the y-axis and the frequency along the $x$-axis can be seen. There were several things to pull from the graph. The first point was that there was a slightly better performance from the circuit at around $10 \mathrm{~Hz}$ than anywhere else in the given frequency range.

The second point was the performance range. From the graph, there was very little performance drop from 0 to $1 \mathrm{kHz}$ which was a much larger range than what was needed. The performance then began to roll off which was what was expected from this amplifier. This graph was also like the gain graph from the specification documents for the amplifier that was used for this design. The desired gain was around 1300 and while the test data and the graph show that it was accomplished with even slightly better performance. Some of those performance results were attributed to the resistors, although they were said to have a tolerance allowing them to be in a range of values. From looking at the graph and the result data, it was confirmed that the amplifier works and could amplify signals on a scale that was needed for the system.

\subsubsection{Antenna analysis}

From the results in the previous section, the data was used to create the graph below to help illustrate how the antennas performed. 


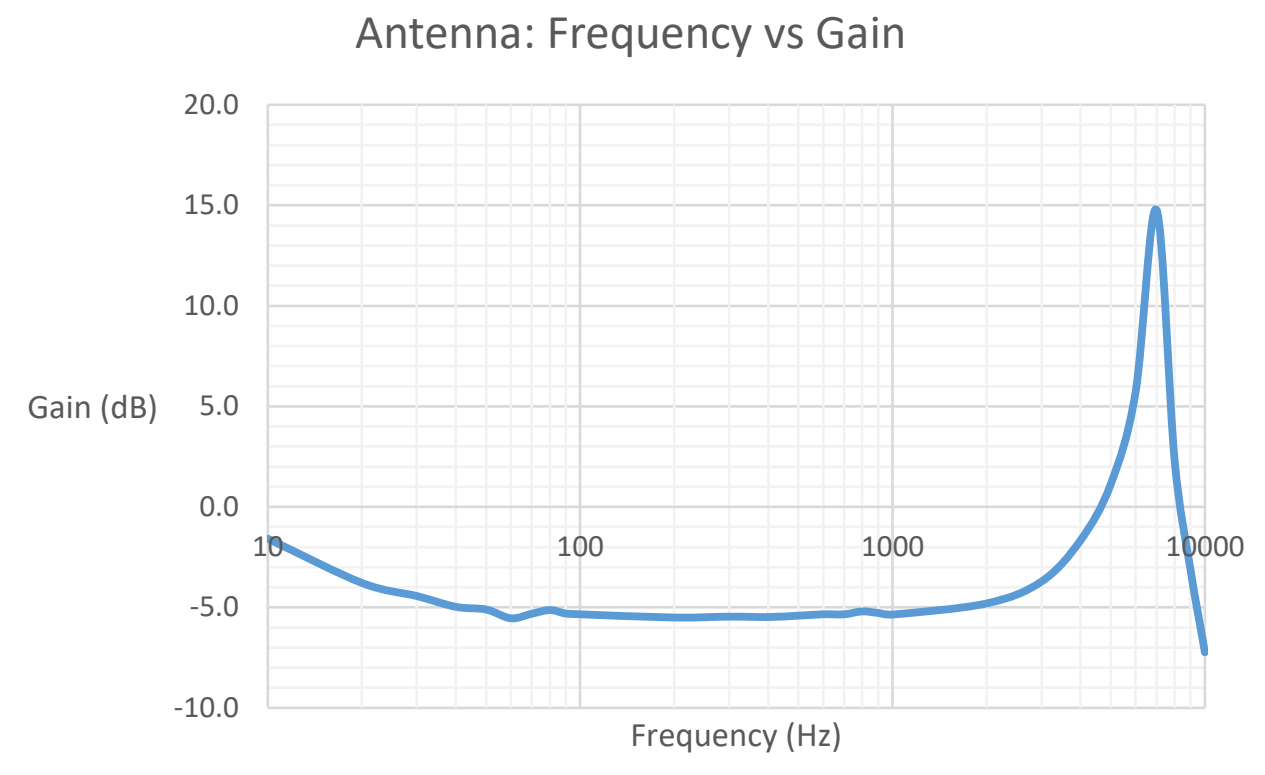

Figure 42 - Antenna frequency vs gain plot full frequency range

Examining the data closer for frequencies less than $1000 \mathrm{~Hz}$ yields the graph below.

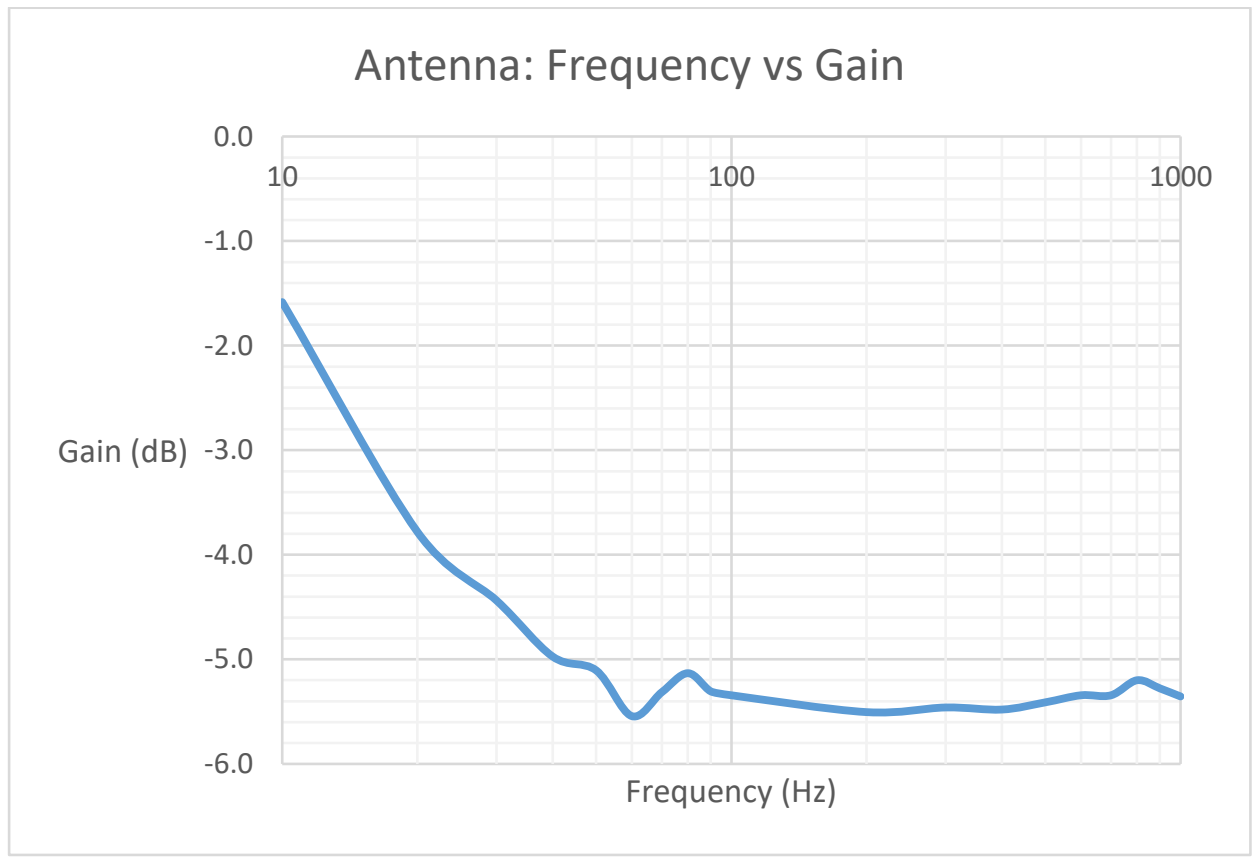

Figure 43 - Antenna frequency vs gain plot $10 \mathrm{~Hz}-1000 \mathrm{~Hz}$

From the graphs, the performance of the antennas was less than perfect. The performance at lower frequencies was not great but worked to test the system and the amplifier. The resonance point was also seen to be around $7 \mathrm{kHz}$ which is slightly different than what was seen from the 
characterization of the large antenna. Overall the antennas performed well enough to be used for the system.

\subsubsection{System analysis}

To analyze the system further the sound card was also tested across the same frequency range so the performance for it could be determined as well. The data that was collected was plotted on the graph below.

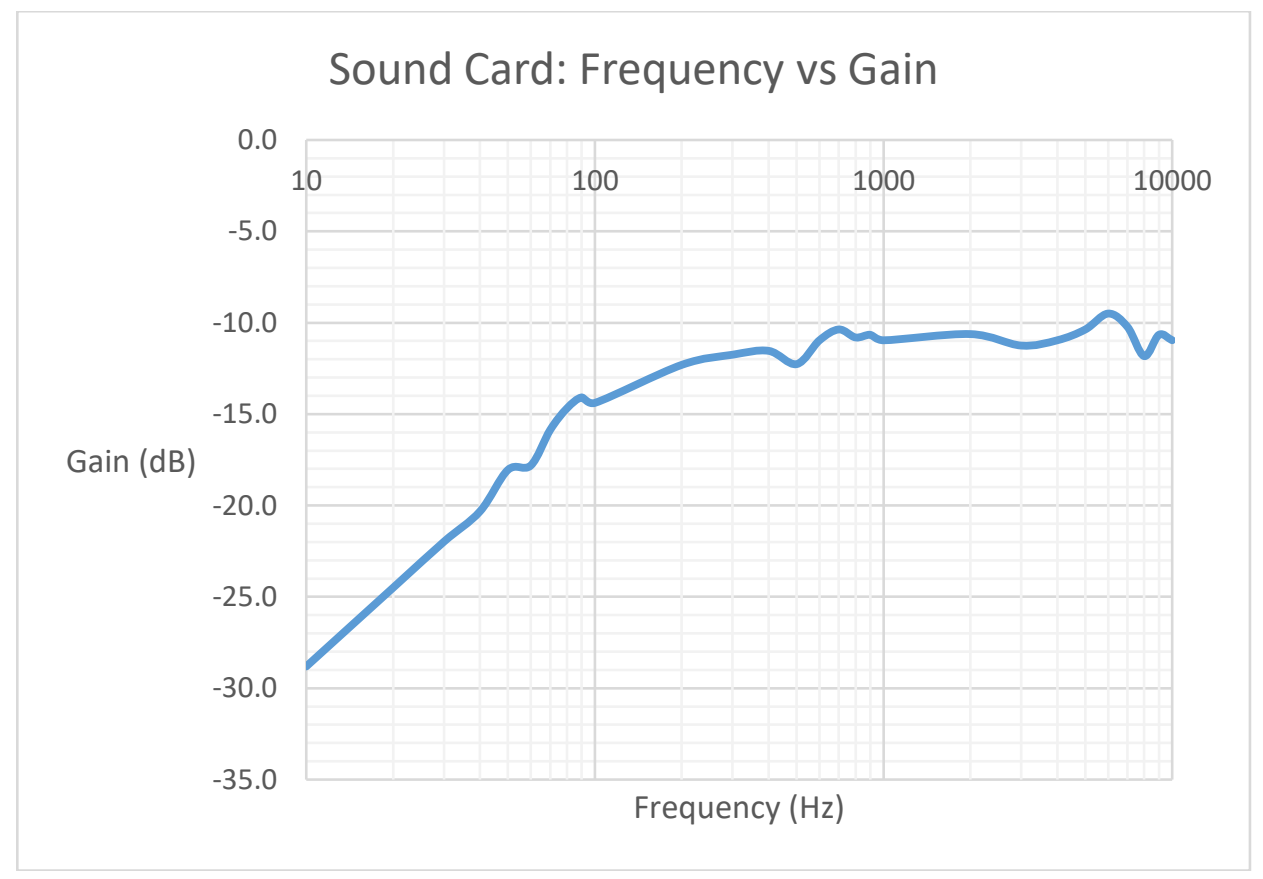

Figure 44 - Sound card frequency vs gain full range plot

By focusing on the results from $1000 \mathrm{~Hz}$ and below the graph below was generated. 


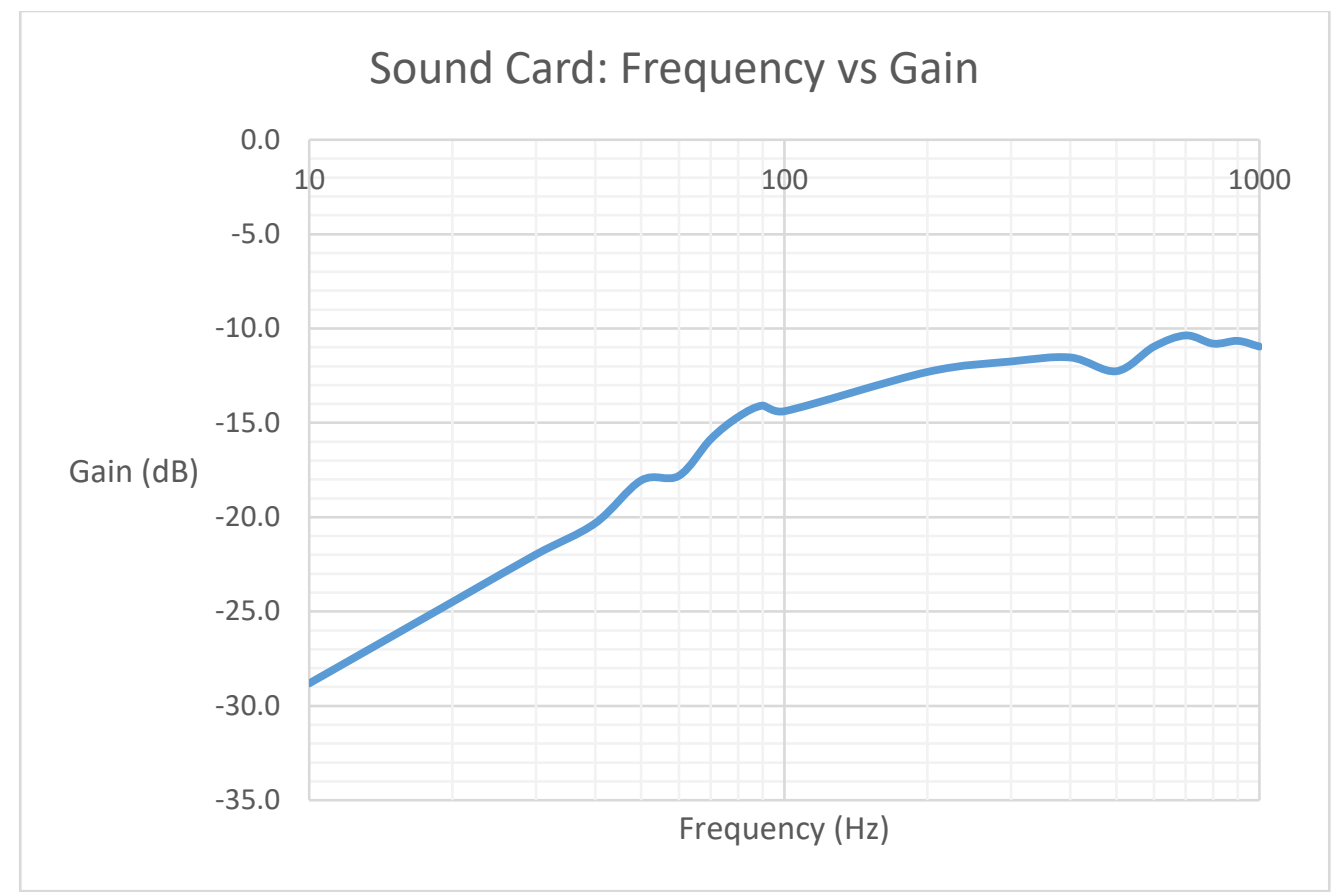

Figure 45 - Sound card frequency vs gain plot $10 \mathrm{~Hz}-1000 \mathrm{~Hz}$

These result show issues below the frequency of interest. The frequency range $5 \mathrm{~Hz}-20 \mathrm{~Hz}$, which is a subset of the range of interest, has almost a $20 \mathrm{~dB}$ attenuation which greatly affects the performance of the system. With this data, the remaining portion of the system test could be analyzed.

From the results section, the data points for the signal level were plotted against frequency so that data could be visualized to show how the system worked. Below is the graph of that data. 


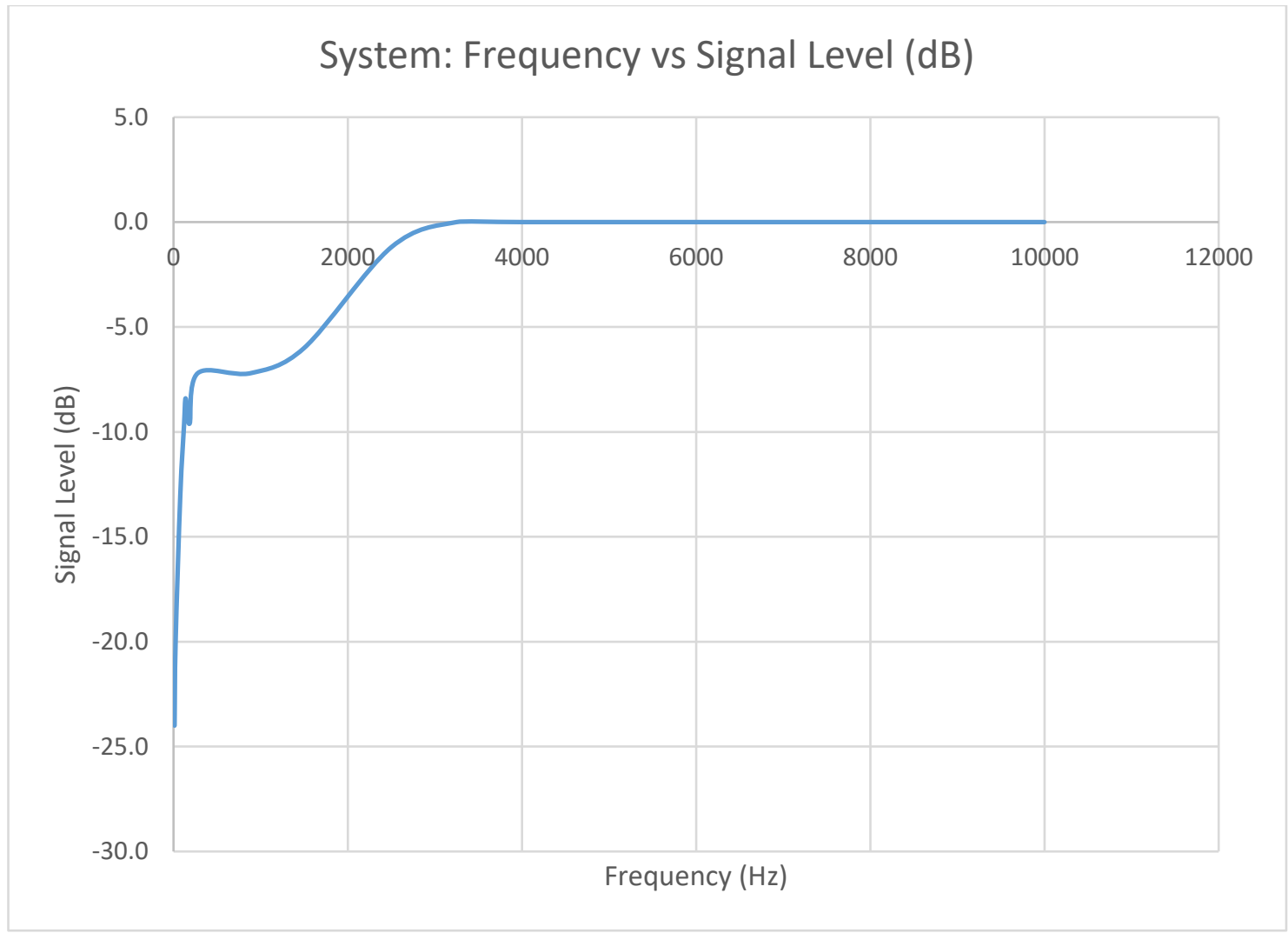

Figure 46 - System output signal level vs frequency plot

Examining frequencies $1500 \mathrm{~Hz}$ and less yielded this graph.

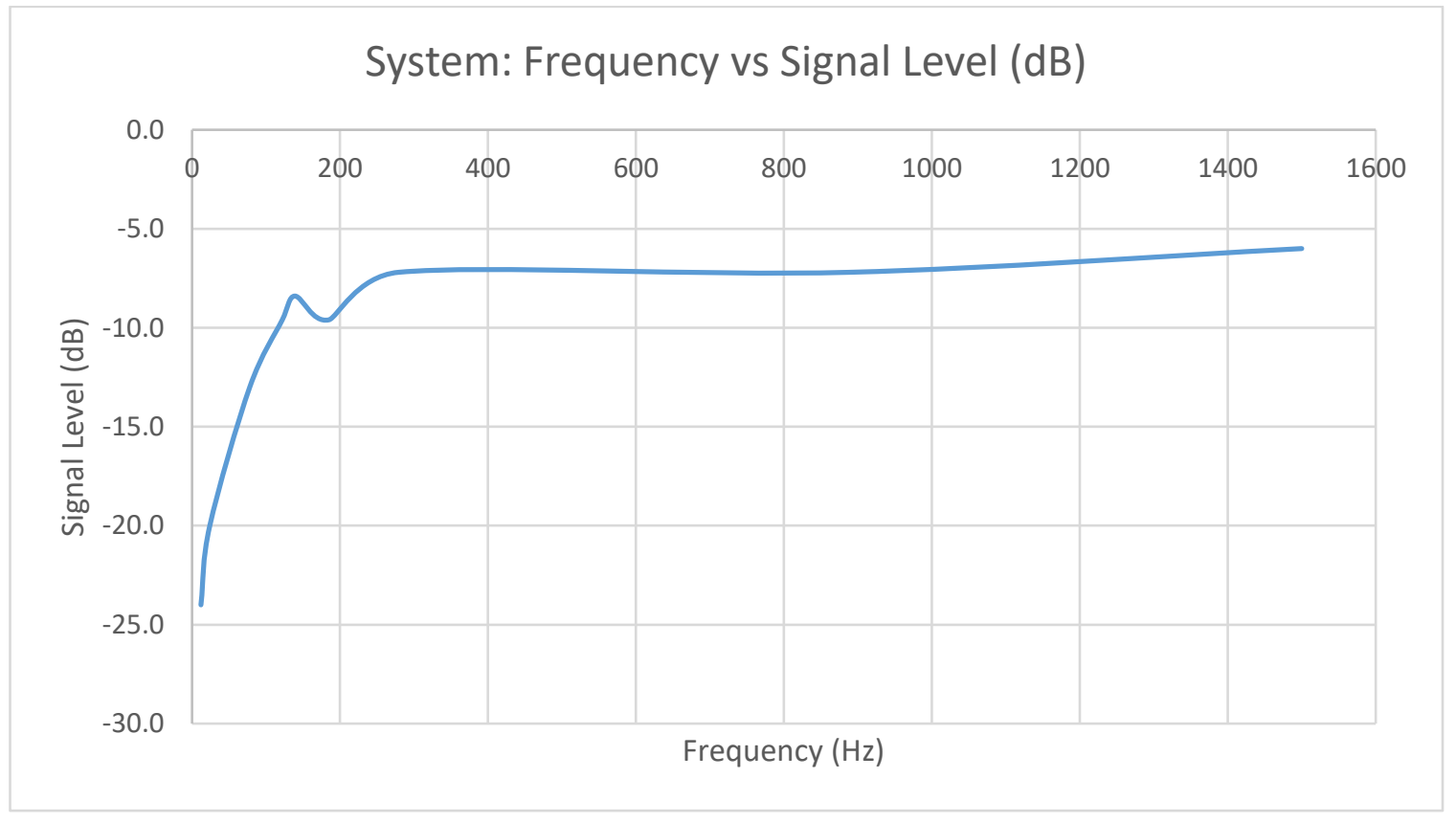

Figure 47 - System output signal level vs frequency plot $1 \mathrm{~Hz}-1500 \mathrm{~Hz}$ 
It was known that with better antennas, the system would have worked better. To break down the graph though, there was a significant increase in signal everywhere, but it was noticed that the signal was not quite as high at the lower frequencies as what was expected. It was already discovered from the amplifier test that the amplifier worked at frequencies that low, and it was also known from testing the antennas that there was less than a $5 \mathrm{~dB}$ attenuation across all frequencies tested. This narrowed the issue down to the sound card. One of the goals for the amplifier was to make sure that the signal level was kept low enough so that it did not have any negative effects on the recording equipment but was strong enough so that it could be received. Overall, it was confirmed that the system performed well and worked to record low-frequency signals. 


\section{CHAPTER 5: SUMMARY OF RESULTS}

\subsection{Summary of Simulation of Amplifier Results}

The simulation of the amplifier showed that it was possible to receive low voltage signals and amplify them to a level that was useful in other parts of the system. The simulations were run for both a signal input source at the desired frequency of $7.8 \mathrm{~Hz}$ and then it was swept across multiple frequencies that helped gain a clearer picture of the how the amplifier performed across a range of frequencies. The input and output of the amplifier were constantly compared to determine what the gain was for the amplifier. From the data, the gain was determined to be around 1600 based solely on the simulation.

\subsection{Summary of Characterization of Antenna Results}

The characterization of the antenna was performed to gain a better understanding of how the antennas performed. The data was used to make informed decisions about how the system would be tested and how they could be best used to replicate a real-world situation where this system would be used. Using an LCR meter two ferrite core antennas were characterized and analyzed. The antennas were measured over a range of $0 \mathrm{~Hz}$ to $10 \mathrm{kHz}$. These values were then plotted so that the resonant frequencies of each antenna could be seen. For the larger antenna with more windings the resonant frequency was at around $8 \mathrm{kHz}$ and for the smaller antenna with fewer windings, the resonant frequency was never reached.

\subsection{Summary of Bench Test Results and Analysis}

The amplifier was the most successful test and most like the simulation results. The test was performed by inputting, approximately, a 1V signal into a voltage divider and dividing the signal down to about $1 \mathrm{mV}$. This signal was then input into the amplifier where the output was measured on an oscilloscope. The test was performed for frequencies between $10 \mathrm{~Hz}-10 \mathrm{kHz}$. From the data, it was determined that the gain of the amplifiers was about $63 \mathrm{~dB}$ for the range of interest $(5 \mathrm{~Hz}-$ $100 \mathrm{~Hz}$ ). The data was plotted and the graph was obtained that was very like the graph obtained during the frequency analysis simulation of the amplifier.

The antennas were connected to the signal generator and the oscilloscope without the amplifier to determine how they responded over the test frequency range. From those results, it was seen that the resonant frequency was around $7 \mathrm{kHz}$ not $8 \mathrm{kHz}$ as was determined during the characterization. There was also a $-5 \mathrm{db}$ attenuation across frequencies from $30 \mathrm{~Hz}-2000 \mathrm{~Hz}$.

The system test was the largest and most complex of all tests that were performed. Two antennas were used one for transmitting and one for receiving. For the receiving side, the antenna was connected directly to the amplifier. From the amplifier, it was hooked up to three different measuring devices. Those devices are the oscilloscope, the digital oscilloscope, and a computer through the line in input running spectrum lab. The results were obtained using the computer and then verified using the other measurement devices. From the test, it was seen that the amplifier 
could amplify a signal that could not be received using just the antenna alone. The table below gives an overview of the design goals and the results from testing.

\begin{tabular}{|l|l|l|}
\hline Design Parameter & Goals & Result \\
\hline Frequency Range & $5 \mathrm{~Hz}-100 \mathrm{~Hz}$ & $10 \mathrm{~Hz}-10 \mathrm{kHz}$ \\
\hline Amplifier Gain & $60 \mathrm{~dB}$ Gain at $<1000 \mathrm{~Hz}$ & $62 \mathrm{~dB}-64 \mathrm{~dB}$ Gain at $<1000 \mathrm{~Hz}$ \\
\hline
\end{tabular}
Table 8 - Results overview

The gain for the system was not quite as high as was anticipated after the amplifier test was run, but this was due to performance at low frequencies from the sound card which resulted in worse system performance. Some attenuation was expected, but more was seen in testing. Overall, the system could receive and view signals that were previously unrecoverable. 


\section{CHAPTER 6: CONCLUSION AND FUTURE WORK}

This thesis described the design of a low-frequency amplifier that could receive low voltage signals and amplify them to a level that could be used by a computer to do further analysis. The design was capable of handling inputs as low as $1 \mu \mathrm{V}$ and amplifying them to a $1 \mathrm{mV}$ level. This thesis also described how to test the amplifier and how to test the rest of the system to determine the performance of the amplifier. The result of this work described a successful design of a low noise amplifier for use in detecting the low frequency and low power signals.

Moving forward, some additional work should be completed to build a complete low-frequency anomaly detector. One of the biggest challenges is building an antenna. Many times, throughout this thesis, the antenna played a role that either hurt the performance of the system or data was unable to be obtained for the antennas. Research was conducted into antenna design, but due to the complexity and scope of the project, an antenna design was not formally chosen. It is believed that a loop antenna would be the best choice for this type of system, though. The antenna needs to be light enough to be carried by hand, it must be able to handle frequencies below $10 \mathrm{~Hz}$ without causing any sort of attenuation, and it needs to be well matched to the amplifier by either design or impedance matching.

Hardware filtering is another area of importance that could be examined. Hardware filtering was examined for this experiment but was decided against due to the complexity of filters at these frequencies. Examining filter techniques and design would beneficial to the amplifier to allow it to only amplify the signals of interest.

Analog to digital conversion is a major area that is missing from this system. A computer sound card is generally used for analog to digital conversion, but these sound cards do not generally have a significant response for signals lower than $20 \mathrm{~Hz}$ so the performance of a typical sound A/D at $7.8 \mathrm{~Hz}$ is not typically known. With a proper analog to digital converter, the system would be able to process with more precision and less attenuation of the input signal.

Another critical element of the system that needs additional work is the software. Only off the shelf software was used for this thesis. An ideal software program would include the display of the frequencies with respect to time, a waterfall display, and be able to analyze frequencies that were less than a few $\mathrm{Hz}$ apart. The program should also be able to do advanced filtering and should be able to process the data in real time to gain an accurate picture quickly of the received signal. This might then be able to give a quick review of what might be underground instead of requiring processing later in a laboratory. 


\section{CHAPTER 7: REFERENCES}

[1] W. Zhu, P. Sun, Y. n. Ma, R. Song, S. w. He and K. H. Liu, "Location Method of Underground Pipeline Monitoring Point Based on Cavity Detection," pp. 75-81, 2012.

[2] R. Siegel, "Land Mine Detection," IEEE Instrumentation \& Measurement Magazine, pp. 22-28, December 2002.

[3] G. A. Tsihrintzis, P. M. Johansen and A. J. Devaney, "Buried Object Detection and Location Estimation from Electromagnetic Field Measurements," IEEE Transactions on Antennas and Propagation, pp. 1742-1744, November 1999.

[4] J. Galejs, "Schumann Resonances," RADIO SCIENCE Journal of Research, pp. 10431055, 8 August 1965.

[5] M. Balser and C. A. Wagner, "Observations of Earth-Ionosphere Cavity Resonances," Nature, pp. 638-641, 1960.

[6] J. J. M. Madrid, J. R. C. Corredera, G. d. M. Vela and F. J. J. Rodriguez, "Detection of Shallowly Buried Objects With Subsurface Radars," pp. 1897-1899.

[7] L. L. Monte, D. Erricolo, F. Soldovieri and M. C. Wicks, "Radio Frequency Tomography for Tunnel Detection," IEEE Transactions on Geoscience and Remote Sensing, pp. 11281137, 2010.

[8] B. P. West, "Tunnel and Subsurface Void Detection and Range to Target Measurement," Idaho National Laboratory, Idaho Falls.

[9] K. E. Gilbert and J. M. Sabatier, "Buried Object Detection," National Center for Physical Acoustics, University, 1987.

[10] Analog Devices, "Instrumentation Amplifier (In-Amp) Basics," 2008.

[11] C. Kitchin and L. Counts, A Designer's Guide to Instrumentation Amplifiers, Analog Devices, Inc., 2006.

[12] Linear Technology, "Single Resistor Gain Programmable, Precision Instrumentation Amplifier," Milpitas, 2011.

[13] PoLabs, "PoScope-Mega 50," 2017. [Online]. [Accessed 29 Janurary 2017].

[14] "Ebay," [Online]. [Accessed 29 January 2017].

[15] DL4YHF, "DL4YHF's Amateur Radio Software," October 2016. [Online]. [Accessed 1 February 2017]. 
[16] N. Cherry, "Schuman Resonances, a plausible biophysical mechanism for the human health effects of Solar/Geomagnetic Activity," Natural Hazards, pp. 279-330, 2002.

[17] B. Carter, "A Differential Op-Amp Circuit Collection," Texas Instruments, 2001.

[18] J. Karki, "Fully differential amplifiers," Analog Application Journal, p. 4, 2000.

[19] T. Kugelstadt, "Getting the most out of your instrumentation amplifier design," Analog Applications Journal, p. 6, 2005.

[20] F. Shimin, Z. Suihua and C. Zhiyi, "A very low noise preamplifier for extremely low frequency magnetic antenna," Journal of Semiconductors, p. 5, 2013.

[21] H. Michlmayr, "Magnetic Antennae For ULF," [Online]. [Accessed 10 February 2014].

[22] M. Sato, G. Fang and Z. Zeng, "Landmine Detection by a Broadband GPR System," pp. 758-760, 2003.

[23] Y. Xu, R. M. Narayanan, X. Xu and J. O. Curtis, "Polarimetric Processing of Coherent Random Noise Radar Data for Buried Object Detection," IEEE Transactions on Geoscience and Remote Sensing, pp. 467-478, March 2001. 